\title{
Resolution enhancement techniques in microscopy
}

\author{
Christoph Cremer ${ }^{1,2, a}$ and Barry R. Masters ${ }^{3,4}$
}

${ }^{1}$ Group Leader, Superresolution Microscopy, Institute of Molecular Biology (IMB), Ackermannweg 4, 55128 Mainz, Germany

2 Kirchhoff Institute of Physics (KIP), University Heidelberg, Im Neuenheimer Feld 227, 69120 Heidelberg, Germany

3 Visiting Scholar, Department of the History of Science, Harvard University, Science Center, No. 1 Oxford Street, Cambridge, MA 02138, USA

4 Visiting Scientist, Department of Biological Engineering, Massachusetts Institute of Technology, 77 Massachusetts Avenue, NE47-220, Cambridge, MA 01239, USA

Received 28 November 2011 / Received in final form 20 November 2012 Published online 23 January 2013

(C) The Author(s) 2013. This article is published with open access at Springerlink.com

\begin{abstract}
We survey the history of resolution enhancement techniques in microscopy and their impact on current research in biomedicine. Often these techniques are labeled superresolution, or enhanced resolution microscopy, or light-optical nanoscopy. First, we introduce the development of diffraction theory in its relation to enhanced resolution; then we explore the foundations of resolution as expounded by the astronomers and the physicists and describe the conditions for which they apply. Then we elucidate Ernst Abbe's theory of optical formation in the microscope, and its experimental verification and dissemination to the world wide microscope communities. Second, we describe and compare the early techniques that can enhance the resolution of the microscope. Third, we present the historical development of various techniques that substantially enhance the optical resolution of the light microscope. These enhanced resolution techniques in their modern form constitute an active area of research with seminal applications in biology and medicine. Our historical survey of the field of resolution enhancement uncovers many examples of reinvention, rediscovery, and independent invention and development of similar proposals, concepts, techniques, and instruments. Attribution of credit is therefore confounded by the fact that for understandable reasons authors stress the achievements from their own research groups and sometimes obfuscate their contributions and the prior art of others. In some cases, attribution of credit is also made more complex by the fact that long term developments are difficult to allocate to a specific individual because of the many mutual connections often existing between sometimes fiercely competing, sometimes strongly collaborating groups. Since applications in biology and medicine have been a major driving force in the development of resolution enhancing approaches, we focus on the contribution of enhanced resolution to these fields.
\end{abstract}

a e-mail: cremer@kip.uni-heidelberg.de; c.cremer@imb-mainz.de 


\section{Introduction}

Because of the importance of enhanced resolution for science, technology and medicine, microscopy is widely regarded an important discovery: advances in optical microscopy correlate well with advances in our understanding of biology, and medicine [Masters 2008; 2009a; 2009b]. Schellenberg edited a book with reprinted papers on the history of optical resolution and its applications to a variety of instruments. These reprinted papers, some of which are translated into English, show that resolution enhancement techniques are important not only for biomedicine, but also for microfabrication and optical lithography [Schellenberg 2004]. One can make the claim that advances in technology and instrumentation drives the advances in research. Microscopy requires both resolution and contrast and in the last decades we have seen substantial advances in the development of both of these areas.

What is fascinating from the historical perspective is that much of the early developments were prescient and often resurfaced in new and exciting forms. In this review we revive some of the seminal advances of the past and integrate them with the exciting modern developments that provide resolution enhancement techniques in optical microscopy.

Each of these resolution enhancement techniques has inherent advantages and limitations. A desirable goal would be to develop a series of microscopes that have spatial and temporal resolutions that span a wide range of scales from atoms to organisms, and down to time scales short enough to allow dynamic analyses. Historically, the development of microscopy has been intimately connected with the biosciences.

Simple optical elements to enlarge the image of objects for an improved structural analysis have been around for thousands of years. Large blocks of glass have been found already in Mediterranean shipwrecks of the 14th century B.C. But for several millennia the technology of glass making and the necessary mechanics to hold the lenses were not sufficiently advanced to allow the construction of high-magnification lens systems, or microscopes. This changed only a few hundred years ago with the construction of the first strongly magnifying optical systems at the end of the 16th and in the early 17 th century. Probably not coincidentally, this invention was made in Italy and the Netherlands, where the textile industry flourished. Microscopes for the first time allowed a detailed analysis of the quality of wool and cloth, a basis of the then "wealth of nations." An early application of a compound microscope to the life sciences is attributed to Galileo Galilei who in the beginning of the 17th century described a bee, the coat of arms animal of the reigning pope Urban VIII. The first widely distributed book on microscopy, however, was published in 1665 by an English physicist. Robert Hooke, Secretary of the Royal Society in the years 16771682, described in his "Micrographia or some physiological descriptions of minute bodies, made by magnifying glasses with observations and inquiries thereupon" observations, including microscopic examination of the tissues of plants. Thanks to the better resolution of his instrument, he discovered small structural units, "cells", or "cellulae". In medieval Latin, "cellula" denoted an empty little room. The fundamental importance of these "little rooms" still remained for another 170 years in the dark, until the first better corrected compound microscopes revealed the fundamental importance of cells (typically with a diameter in the $10-20 \mu \mathrm{m}$ range) for all living systems.

The early microscopists of the 17th century observed not only wool, cloth, cork, small organisms in water droplets or sperm at enhanced resolution. The most famous of them, together with Robert Hooke, the Dutchman Antonie van Leeuwenhoek (16321723) was the first to use his simple single lens microscope with a short focal length (a few $\mathrm{mm}$ ) and a resolution down to the $\mu \mathrm{m}$-range to observe for the first time bacteria [Masters 2008]. Microscopes at that time had many technical problems making 
the sharp, clear observations of the micro-world extremely difficult. The systematic observation of bacteria was suspended until the 19th century when multi-element, robust and "user friendly" microscope systems with better lighting and good chromatic and monochromatic corrections could be manufactured. The usable optical resolution of these microscopes was approximately 1 micron or $1 / 1000$ millimeter. Although this was not much better than what Leeuwenhoek had achieved 170 years earlier, there were large differences in the practical usefulness: instead of a single tiny lens that one had to keep very close to the eye for observation, Matthias Schleiden (1804-1881), the founder of the cellular theory of life, used a compound microscope of the French company Oberhäuser (Paris/F), allowing precise focusing of the object. Furthermore, Schleiden's microscope had an optimized illumination system. The mechanical stability was supported by a lead-filled base; to achieve a precise focus, a spring mechanism was used [Cremer 2011]. The preparations were at this microscope on glass slides that were clamped with steel springs on the stage, just as with conventional systems today. Consequently, these microscopes have a resolution down to the $\mu \mathrm{m}$ range already contained various elements (high quality optical components; appropriate illumination; high mechanical stability) essential also for the modern development of enhanced resolution. Such microscopes were probably the best that in the first half of the 19th century was available in the field of high resolution optics.

In the year 1846, with the help of Matthias Schleiden, Carl Zeiss (1816-1888) opened a small optical workshop in Jena and began to manufacture microscopes. These were then constructed according to empirical rules. Carl Zeiss was the first to recognize the importance of optical theory for the construction of high-power microscopes. Therefore, in 1866 he committed the Jena physicist Ernst Abbe (1840-1905) to perform quantitative numerical calculations for the development of further improved microscope systems [Masters 2007]. By the 1880s, the microscopes built by Zeiss and Abbe were the best in the world. The new microscopes from Zeiss optics and some other leading manufacturers enabled the founding of modern cell based medicine and microbiology. For example, the new microscopes with very much improved mechanical stability, illumination, optical error correction and improved resolution allowed for the first time a detailed analysis of many bacterial pathogens such as anthrax, tuberculosis, cholera, and plague. By this, diagnosis, therapy and hygiene were put on completely new foundations, with decisive consequences for the life conditions of mankind. In addition, microscopes were used more and more to increase knowledge also in other fields, such as geology, or even physics. The improved microscope systems manufactured by Zeiss had a useful optical resolution down to 0.2 microns. As good as the new high-power microscopes were, they had a serious problem: structural details of bacteria (size in the $\mu \mathrm{m}$ range) and cells could only be detected if they were larger than about 0.2 microns. Despite all attempts to improve this, it was not possible to overcome this limit of the "conventional" light microscopy.

To summarize, so far historical evidence demonstrates the utmost importance of improved resolution in science, technology, and in particular in biology and medicine.

\section{What is resolution?}

In common language, the word 'resolution' may generally be defined as the "action or process of separating or reducing something into its constituent parts" (The American Heritage Dictionary of the English Language). Accordingly, the application of this general meaning in optics may be understood as the power of a microscope system to discriminate the constituent parts of an object down to a certain level of distinction. Historically, the first clearly formulated general resolution criteria have been those of Ernst Abbe (1873) and Lord Rayleigh (1896). In addition, also some other special 
resolution criteria have been put forward to describe the principal goal of resolution, "to discriminate the constituent parts of the object." For example, resolution criteria based on the Nyquist theorem may be very useful to describe the power of a microscope approach to analyze the structure of a completely unknown object. In other cases, it may be of great importance for the proper discrimination of the constituent parts of the object to give a measure for the smallest detectable size (extension) of such a constituent part; or to give a value for the smallest detectable observation volume. Such a criterion based on a three dimensional (3D) measure appears to be particularly useful to describe the power of an optical instrument to discriminate the constituent parts in a $3 \mathrm{D}$ structure in the biosciences, such as an individual cell. Hence, there are different criteria of resolution. Some of these criteria (such as two-point resolution, point-spread-function and Fourier based definitions) are generally accepted to describe the performance of optical systems. Others may be useful for special applications, in particular in the life sciences. In this historical report on the techniques of resolution enhancement, we shall list the various criteria and abstain from a decision what should be 'the right criterion'. In addition, we shall also abstain from the concept of a 'best resolution:' the best resolution is the resolution which allows to the answer the problem posed. According to the problem, this may be a criterion based on twopoint resolution, a PSF, or Fourier based concept; it may be the Nyquist theorem applied to microscopy, the observation volume, or the size resolution.

\subsection{Resolution criteria}

\section{Two-point resolution}

There are various criteria of two-point resolution for the resolving power of an optical instrument [Dekker and van den Bos 1997]. Experimentally, the resolving power of an instrument depends on the shape of the object. We refer to resolution criteria instead of definitions. The concept of resolving power involves the ability of an imaging device, for example the eye or an optical instrument, to discriminate two point sources of light, of equal intensity, as two distinct points. For a microscope this is the ability to resolve two points separated by a minimal distance. This minimal distance is often termed the resolution. For example, with a resolution of $100 \mathrm{~nm}$, two luminous point objects, separated by more than $100 \mathrm{~nm}$, will be imaged as two distinct points; two luminous objects with a smaller separation will not be imaged as two distinct points. For a standard optical microscope with visible illumination, diffraction limits the spatial resolution to about $0.2 \mu \mathrm{m}$ in lateral direction and about $0.6 \mu \mathrm{m}$ in the axial direction.

\section{Resolution based on the point spread function}

In the Rayleigh theory, two self-luminous point sources in a sufficiently large distance are represented by two well separated diffraction patterns (Airy disks) [Rayleigh 1880a, 1880b, 1896, 1899]. The position of their maxima and hence their distance can be determined as long as the maxima are well separated from each other. It is evident that the sharper these Airy discs are (i.e. the smaller their diameter) the smaller is the detectable distance between them and hence the better the two-point resolution. This idea has been generalized in the resolution criterion based on the full-width-athalf-maximum (FWHM) of the point-spread-function. Experimentally, the PSF is the normalized intensity image of a self-luminous point source (e.g. a normalized Airy disc); its FWHM is the diameter at one-half of the maximum intensity (giving a 
measure of the 'sharpness'). Depending on the optical system, the FWHM has different values in different directions in the object plane (coordinates $x, y$ ) and along the optical axis $(z)$. Typically, two object points can be discriminated (resolved) from each other if their distance is larger than the FWHM. This criterion has been amply used to describe e.g. the resolution power of focused nanoscopy methods.

\section{Fourier based resolution criteria}

Ernst Abbe [Abbe 1873] developed his resolution criterion on the assumption that the resolution limit of an object as the finest detail to be discriminated may be denoted by the finest grid (in terms of lines $/ \mathrm{mm}$ ) which can be imaged, i.e. by the smallest grid-to-grid distance which can still be detected by the optical system. The theoretical justification for this was the possibility discovered by Jean Baptiste Fourier (1768-1830) to mathematically describe any continuous object as the superposition of harmonic functions.

McCutchen [McCutchen 1967] used Fourier analysis to analyze the effect of apertures or stops on an imaging system. The Fourier theorem states that any periodic function $f(x)$ can be expressed as the sum of a series of sinusoidal functions.

$$
f(x)=\frac{1}{2} C_{0}+\sum_{n=1}^{\infty} C_{n} \cos \left(n k_{0} x+\alpha_{n}\right) .
$$

The $n$ s are called the orders of the terms and are harmonics, and $k_{0}=\frac{2 \pi}{\lambda}$.

Each term in the above series has two Fourier coefficients, an amplitude $C_{n}$, and a phase angle $\alpha_{n}$.

McCutchen noted that the physical principle is similar to the local oscillator in a super-heterodyne radio receiver; both shift the signal frequencies into the bandpass, spatial or temporal, of a filter. He then ruled out superresolution microscopy based on transmitted light; because of the difficulty of placing a stop much nearer than 100 wavelengths from the object. And he then examined the reflected light microscope in which the point source of light has a diameter much smaller than the wavelength and is scanned over the object. The physical principle is as follows for a fluorescent object (incoherent emission): the object is illuminated with the smallest possible diffraction image of a point source. The image is the convolution of the spatial spectrum of the object with the autocorrelation function of the illuminating pupil. This increases by $2 / \lambda$, where $\lambda$ is the wavelength of the incident light, the largest spatial frequency that forms the image. Such a microscope would be superresolving since there is a gain of more than a factor of 2 .

Convolution, correlation, and autocorrelation are common mathematical operations in optics. We mathematically define the convolution of two real functions $f$ and $g$ as:

$$
h(x)=\int_{-\infty}^{+\infty} f\left(x^{\prime}\right) g\left(x-x^{\prime}\right) d x^{\prime}
$$

This is sometimes written as:

$$
h(x)=f(x) \otimes g(x) .
$$

The correlation function is mathematically defined as:

$$
h_{\text {corr }}(x)=\int_{-\infty}^{+\infty} f\left(x^{\prime}\right) g^{*}\left(x^{\prime}-x\right) d x^{\prime} .
$$

Which is the convolution of $f(x)$ and $g^{*}(-x)$. 
The auto-correlation function, is similar to the correlation function, but we set $h \equiv f$, then:

$$
h_{\text {auto-corr }}(x)=\int_{-\infty}^{+\infty} f\left(x^{\prime}\right) f^{*}\left(x^{\prime}-x\right) d x^{\prime} .
$$

\section{Additional criteria of resolution}

\section{Nyquist Theorem based Resolution criteria}

Originally, the Nyquist-Shannon sampling theorem has been derived from information theory and is used to evaluate the transmission of signals. In a modified version, it is applied also to describe basic requirements to obtain an image of a given resolution. It is a consequence of the assumption that an object (continuous in time or space) is represented by the superposition of harmonic functions of various frequencies; the highest transmittable frequency determines the resolution. The frequencies have to be transmitted by sampling, i.e. the process of converting a signal into discrete numbers. This means that to transmit the spatial "cut-off" frequency which determines the resolution, one has to transmit its coordinates at a series of points spaced with the double frequency. For example, for a microscopic resolution of structural details down to $0.2 \mu \mathrm{m}$, it is required to transmit a spatial frequency of at least 5 lines $/ \mu \mathrm{m}$. For that, one needs a density of transmitted object points of double this frequency, i.e. of $10 / \mu \mathrm{m}$.

Obviously, the applicability of this criterion depends on the structure to be resolved. For example, to determine the resolution of a long, highly folded microtubule fiber in a cell, or of a similar polymer on a surface, the Nyquist criterion is very useful. However, in other cases other criteria, such as two-point resolution may be much more indicated. For example, to determine to what extent a point object A (e.g. a short DNA sequence in the nucleus of a cell) is adjacent to a point object B (e.g. another short DNA sequence, or a specific protein), the information required is to identify the positions of A and B independently from each other, and to determine the distance. If there are e.g. only two point objects A, B with a distance of $0.2 \mu \mathrm{m}$ in the field of view, it makes no experimental sense to require the existence of at least 10 distinguishable objects per $\mu \mathrm{m}$ (corresponding to the Nyquist conditions for the transmittance of a spatial frequency of 5 lines $/ \mu \mathrm{m}$, corresponding to a resolution of $0.2 \mu \mathrm{m})$ to perform the measurement. In such a case, it is completely sufficient if the diffraction patterns generated by the two objects $\mathrm{A}$ and $\mathrm{B}$ can be distinguished from each other.

It may be noted that similar considerations hold also in astronomy: to resolve a binary-star system, it is completely sufficient to register the two diffraction patterns separately from each other; the Nyquist theorem is not applicable. The name recently given by the Carl Zeiss Company to their enhanced resolution ELYRA microscope system based on single molecule localization may allude to this point of view: Beta Lyrae is a binary star system approximately 960 light-years away in the constellation Lyra.

\section{Resolution based on the observation volume}

Historically, the resolution of the light microscope referred to its power to discriminate small structures situated in the object plane; the discrimination of object details along the optical axis $(z)$ was not considered, due to the impracticability to generate sufficiently sharp images as a function of $(z)$. This changed when deconvolution based reconstruction algorithms and confocal laser scanning fluorescence microscopy 
techniques were introduced in the 1980s. To describe the 3D resolution power of a microscope system, the FWHM of the PSF in the 3 spatial directions $\left(\mathrm{FWHM}_{x}\right.$, $\mathrm{FWHM}_{y}, \mathrm{FWHM}_{z}$ ) may be used. In this way, however, it may be difficult to compare the resolution power of different systems; e.g. how to compare the 3D resolution power (i.e. its usefulness to discriminate structures in $3 \mathrm{D}$, e.g. in a cell) of a system with $\mathrm{FWHM}_{x}=\mathrm{FWHM}_{y}=200 \mathrm{~nm}$ and $\mathrm{FWHM}_{z}=100 \mathrm{~nm}$ ) with a system with $\mathrm{FWHM}_{x}=\mathrm{FWHM}_{y}=80 \mathrm{~nm}$ and $\mathrm{FWHM}_{z}=600 \mathrm{~nm} ?$

To facilitate such comparisons, it has been proposed to use the 'observation volume' $\left(V_{\text {obs }}\right)$ as an additional criterion [Hell and Stelzer 1992a]. This may be defined as the volume enclosed by an isosurface of the 3D-PSF, e.g. as the ellipsoid volume given by the half values of the FWHMs $\left(\mathrm{V}_{\mathrm{obs}}=4 / 3 \pi * \mathrm{FWHM}_{x} / 2 * \mathrm{FWHM}_{y} / 2\right.$ * $\mathrm{FWHM}_{z} / 2=\pi / 6 * \mathrm{FWHM}_{x} * \mathrm{FWHM}_{y} * \mathrm{FWHM}_{z}$ [Lindek et al. 1994].

\section{Resolution based on size}

In many applications of microscopy in the biosciences, it is important to determine the size of small objects, i.e. their extension in space. For example, a large number of the 'biomolecular machines' maintaining the life of a cell have a diameter in the range of $100 \mathrm{~nm}$ and below. The smallest size which can be determined by a microscope system may be called size resolution. Generally, the size resolution is intimately connected with the FWHM of a system [Baddeley et al. 2010a]; however, in some specially dedicated microscope systems there may be a large difference between these two resolution criteria [Failla et al. 2002a,b].

\section{First concepts of superresolution}

Toraldo di Francia, is credited with the introduction of the concept of superresolution of images. In his 1955 paper he defined superresolution as detail finer than the Abbe resolution limit.

Toraldo di Francia introduced the technique of pupil plane filters as a method to increase the resolution of an imaging system beyond the diffraction limit. The principle was to use two concentric amplitude and or phase filters in the pupil of the imaging system. He also demonstrated the existence of evanescent waves which are today used in total internal reflection microscopy (TIRFM) where the exponentially decaying evanescent radiation field of the exciting light excites a very limited region of the specimen's fluorescence; typically the surface of cells.

Toraldo di Francia in an article on resolving power and information wrote: "Resolving power is not a well-defined physical quantity," [Toraldo di Francia 1955]. He showed that enhanced resolution, that is resolution of visible light microscopy beyond the conventional limits of diffraction (about $200 \mathrm{~nm}$ in the object plane and $600 \mathrm{~nm}$ along the optical axis), is possible if there is prior knowledge about the object being observed. Without prior knowledge about the object there can be no resolution gain. In his 1952 paper published in Italian, he showed the effects of superresolving pupils. In general the resolution enhancement was only obtained for the central part of the field and what was lost was resolution in the peripheral parts of the field.

A decade later Charles W. McCutchen published an article in which he states that in principle one can construct a superresolving optical system that can resolve details finer than the diffraction limit. He then asks the question: "can the diffraction limit for a lens of large numerical aperture be beaten?"

Ernst Abbe coined the term "numerical aperture." In microscopy, the numerical aperture, or $N A$, of a microscope objective is written as: $N A=n \sin \theta$, where $n$ is the refractive index as defined above, and $\theta$ is one-half of the angle of the cone of light that can enter the microscope objective. The angular aperture of the microscope objective is twice the angle $\theta$. 
McCutchen then asks if detail smaller than half of the wavelength of light (this limit in resolution follows from the Abbe diffraction theory of microscopic image formation, see section 3.1) be made visible? He answers yes, but only in specialized and probable limited applications. McCutchen asks the prescient question: can superresolution really beat the ultimate Abbe resolution limit for a lens with an acceptance solid angle of $2 \pi$ steradians. Perhaps that question stimulated the thinking of the inventors of "4Pi" optical microscopes that use two opposing microscope objectives with the specimen between them [Hell 1990b; Hell and Stelzer 1992a,b; Hell et al. 1994a,b; Hänninen et al. 1995].

In another set of interesting but difficult to understand publications W. Lukosz reviewed optical systems with resolving powers that exceed the classical diffraction limit [Lukosz 1966, 1967]. Prerequisites for understanding these two papers include a sound knowledge of Fourier transforms and a good knowledge of optical coherence theory. Lukosz makes two assumptions in his analysis: linearity and space invariance. The linearity condition follows from the linearity of Maxwell's equations. The second condition of space invariance holds that all points in the object field are equivalent; in other words the amplitude distribution in the image of a point source (point-source spread function) "should not change as the source explores the object field."

First, he redefines the limit of resolution of a coherent optical system due to diffraction as stated by Abbe [Abbe 1873]. Optical systems transfer a limited band of spatial frequencies; the bandwidth depends on the angular aperture of the system and the wavelength of the light. Lukosz then states that for a specific optical system, it is not the bandwidth of the transferred spatial frequencies, but the number $N$ of degrees of freedom of the optical message transmitted that is constant. The number $N$ is given by: the product of object area times optical bandwidth, times 2 which is the number of independent states of polarization of the light, times the number of temporal degrees of freedom.

In his invariance theorem, Lukosz showed that the spatial bandwidth of the system is not constant and it can be increased over the classical limit by reducing the number of degrees of freedom of the information that the system can transmit. As Lukosz stated: "A new theorem on the ultimate limit of performance of optical systems is established: not the bandwidth of the transferred spatial frequencies but only the number of degrees of freedom of the optical message transmitted by a given optical system is invariant. It is therefore possible (a) to extend the bandwidth by reducing the object area, (b) to extend the bandwidth in the $x$ direction while proportionally reducing it in the $y$ direction, so that the two-dimensional bandwidth is constant, (c) to double the bandwidth when transmitting information about one state of polarization only, and (d) to extend the bandwidth of transferred spatial frequencies above the classical value by reducing the bandwidth of the transferred temporal frequencies. In all of the described optical systems there are assumed to have linear and approximately space-invariant imaging properties.

To achieve this, the optical systems are modified by inserting two suitable masks (generally gratings) into optically conjugate planes of object and image space. The transfer and spread function of the modified systems are calculated for the case of coherent illumination [Lukosz 1966]. In this paper the author only considers coherent illumination, but the "superresolving systems" also work when the object is illuminated with either partially coherent or incoherent light.

The limits of resolution in the Abbe and Rayleigh theories (about $0.2 \mu \mathrm{m}$ in lateral direction and about $0.6 \mu \mathrm{m}$ in the direction of the optical axis for visible light) are based on specific assumptions: a single objective lens, single photon absorption and emission in a time independent linear process at the same frequencies, and uniform illumination across the specimen with a wavelength in the visible range. If these assumptions are negated then enhanced resolution is feasible. 
This last statement was first put forward by Abbe, and is so important that we quote from his paper. In his famous contribution on the fundamental limits of optical resolution achievable in (far field) light microscopy (Abbe 1873), Abbe stated on page 468 of his 1873 publication: that the resolution limit of about half the wavelength used for imaging is valid only "... so lange nicht Momente geltend gemacht werden, die ganz ausserhalb der Tragweite der aufgestellten Theorie liegen..." (. . as long as no arguments are made which are completely beyond the theory stated here..."). It is precisely by altering the experimental conditions from those stated by Abbe that enhanced resolution in the optical microscope is achieved. The contributions of the various research groups to this achievement are described in Section 5.

\subsection{The role of diffraction in image formation}

An understanding of diffraction theory is central to any understanding of its crucial role in image formation in the microscope. Born and Wolf in their classic book provide a comprehensive discussion of the elements of diffraction theory [Born and Wolf 1980]. The reader may find the book Optical Physics, 4th Edition to be an alternative useful description of the analysis of diffraction theory of light [Lipson, Lipson, and Lipson 2010]. The authors point out that in the region of focus of a lens the geometrical model of electromagnetic energy propagation is no longer adequate; in its place a complete theory of diffraction is required.

Francesco Grimaldi in the 1600s is credited with the origin of the term diffraction which refers to the deviation of light from rectilinear propagation.

In 1818 Augustin-Jean Fresnel (1788-1827) demonstrated that the use of Christiaan Huygens' (1629-1695) construction and the principle of interference (when two or more wavelets interact) could explain the alternating light and dark bands observed in diffraction phenomenon. The approach used by Huygens, subsequently called the Huygens' construction, was to consider a wavefront as a new virtual source of a spherical wave. He called the new spherical waves "wavelets." The new wavefront was formed from the envelope of the Huygens' wavelets. Note that in an anisotropic medium, the spherical Huygen's wavelets take the form of ellipsoids. While the Huygens' construction allowed one to describe the refraction of light, it failed to account for the phenomenon of diffraction of light.

Fresnel used Huygens' construction in which the points on the wavefront are a source of a secondary disturbance and the wavefront at later times is just the envelope of these wavelets. But Fresnel added the critical assumption that the secondary wavelets can interfere with each other and that accounted for the interference of light. Fresnel assumed that the secondary spherical wavelets have the same frequency as their sources on the wavefront. He calculated the amplitude of the optical field outside the wavefront at an instant of time as the superposition of all the wavelets by taking into consideration all of their amplitudes and their relative phases. Fresnel was able to calculate the light distribution of a variety of diffraction patterns. His theory took into account the phases of the secondary Huygens' wavelets, and thus accounted for their interference.

Then in 1882 Gustav Robert Kirchhoff's (1824-1887) theory of diffraction provided a new mathematical basis for the Huygens-Fresnel theory of diffraction phenomenon; this is based on the Huygens-Kirchhoff diffraction integral.

Joseph von Fraunhofer (1787-1826) and Fresnel theories provide some useful approximations to the Kirchhoff theory; when the quadratic and higher-order terms can be neglected we have the Fraunhofer diffraction, and when these terms cannot be neglected the mathematical formulation is termed Fresnel diffraction. Typically in optics we can use the mathematical approach of Fraunhofer diffraction. 
Many problems in optics can be solved by invoking the scalar-wave theory of diffraction which assumes that the amplitude and the phase of an electromagnetic wave can be described by a scalar variable. It specifically neglects the polarization of the electromagnetic waves.

A complete description of the electromagnetic field requires the specification of the magnitude of the field vectors and their polarization as functions of position and of time [Born and Wolf 1980]. The authors show how the vector theory can be replaced with a scalar theory based on the definition of the measurable quantity intensity (I) which is the time average of the energy flow across a unit area that contains the electric and the magnetic vector in unit time. In the scalar wave approximation the effects of polarization are neglected, and the key approximation is that both the amplitude and the phase of the electromagnetic wave can be described by a scalar variable. The authors then develop the scalar theory of diffraction (Fraunhofer diffraction) for the special cases of apertures: rectangular aperture of a slit, and the case of the circular aperture. The latter case is important for the understanding of diffraction from a lens.

George Biddell Airy in 1828 became a Professor of Astronomy and Experimental Philosophy in the University of Cambridge and director of the new Cambridge Observatory, and subsequently in 1835 he was appointed as the Astronomer Royal and he held that position until 1881.

In 1835 Airy developed his formula for the diffraction pattern, called the Airy disk, which is the image of a point source of light in an aberration-free optical system. The special case of the Fraunhofer diffraction of a circular aperture is given an eponymous name: the Airy pattern [Airy 1835]. Airy computed the analytical form of the diffraction pattern from a point source of light (a distant star) as images by the circular lens of the objective. He showed that the image of a star is not a point but a bright circle that is surrounded by a series of bright rings.

With respect to image formation in a microscope the finite aperture of the objective results in diffraction effects. The image of a point source of light in the object or specimen plane is not imaged to a point of light in the image plane by the microscope objective. The diffraction image is formed in the diffraction plane by the microscope objective. The observed Airy diffraction pattern is the Fraunhofer diffraction pattern that is formed by the exit pupil of the microscope objective. The central bright disk in the Airy diffraction pattern is known as the Airy disk. The radius of the Airy disk from the central maximum intensity peak to the first minimum is given as: $r=0.61 \frac{\lambda}{N A}$, where $\lambda$ is the vacuum wavelength of the light, and $N A$ is the numerical aperture.

\subsection{The development of the two-point resolution concept}

In this section we describe how two-point resolution was developed in astronomical telescopes for the case of two luminous point objects and inserted in the context of the diffraction theory of light. Many astronomical objects can be taken as luminous point sources due to their distance from the imaging system. Luminous point objects occur also in microscopy. In both cases the finite diameter of the imaging lens system results in a point being imaged as the diffraction pattern (point spread function) of the system's aperture.

Two-point resolution refers to an optical system's ability to resolve two nearby point sources of light of equal brightness. The criteria of Rayleigh, John William Strutt, 3rd Baron Rayleigh, (1842-1919) and C. M. Sparrow follow. Note that the Rayleigh criterion of resolution (Figure 1) is based on the assumption that the twopoint sources are incoherent with respect to each other [Rayleigh 1880a, 1880b, 1896, 1899]. The Sparrow criterion can also be used for coherent light sources. 

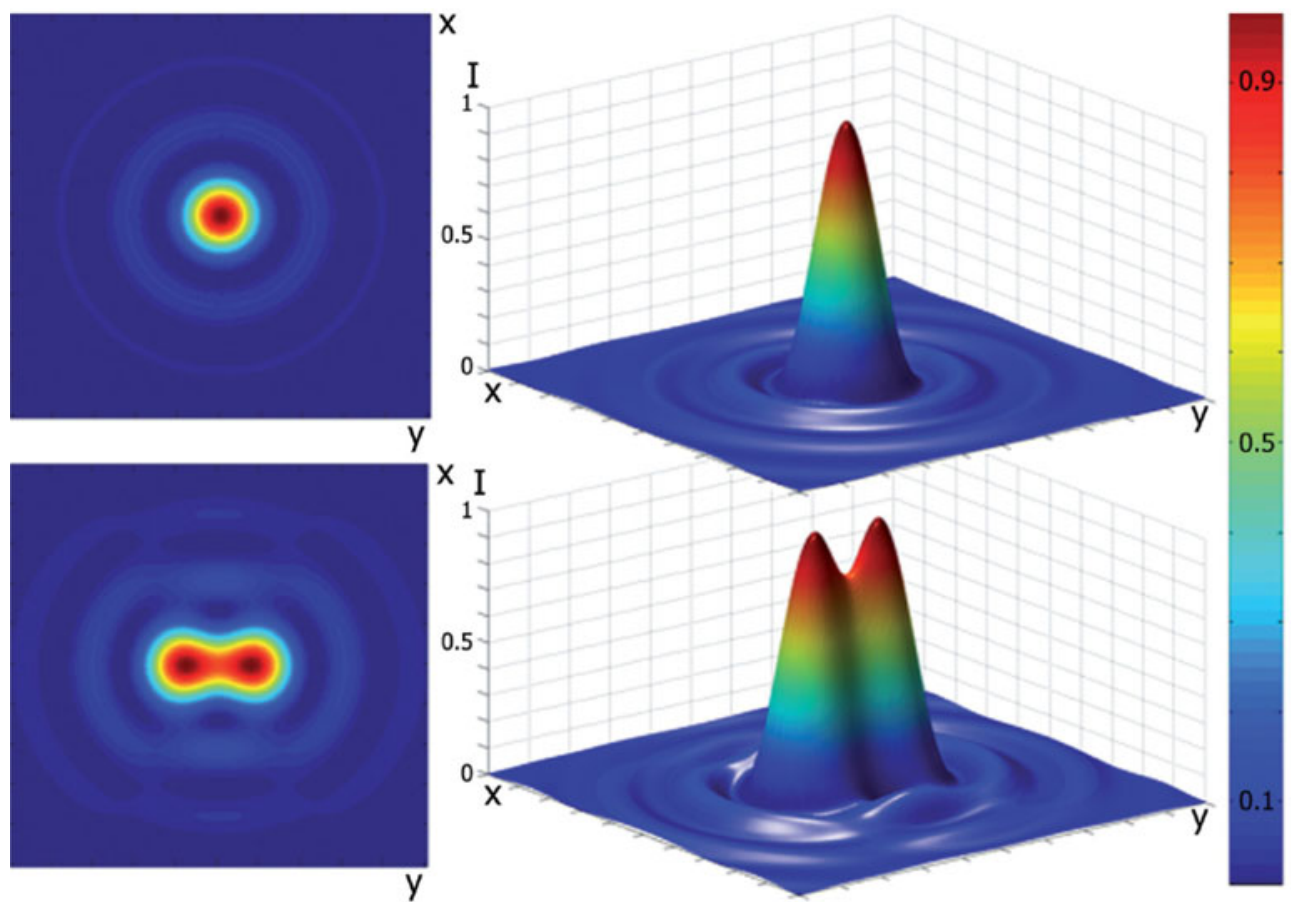

Fig. 1. Diffraction pattern (Airy disc) produced by the fluorescence emission of a single self luminous point source, such as a fluorescent molecule (top), and of two overlapping diffraction patterns (Airy discs) produced by two adjacent point sources (bottom). The smallest resolvable distance between the two maxima has been defined as the optical (twopoint) resolution (Rayleigh criterion). Reprinted with permission from Physik in unserer Zeit (Wiley-VCH) [Cremer 2011].

The Rayleigh criterion is based on the assumption that the human visual system requires a minimal contrast to separate two luminous, incoherent point sources in a composite intensity distribution. The two points of equal brightness are imaged by a diffraction limited optical system. Due to the finite size of the optics in an optical system a point source of light is not imaged as a point, but as the diffraction pattern of the system's effective aperture. This diffraction pattern is called the point spread function. We may sample an extended object as a collection of point sources. If the light sources are incoherent, their intensities are added. The image is the convolution of the object intensity and the point spread function (PSF).

In the Rayleigh resolution criterion there are two points of light that are separated by a small angle. Rayleigh's definition is the following: the two points are resolved if the central maximum of the diffraction pattern from the first point coincides with the first zero of the second point's diffraction pattern.

If there is no zero in the vicinity of the central maximum, Rayleigh proposed that the resolution is given by the distance for which the intensity at the central minimum in the combined image of the two equal point sources is $80 \%$ of the maxima of intensity on each adjacent side. Therefore, if the intensity profile shows a dip in the middle that is higher than $80 \%$ of the maximum intensity, then the two points cannot be resolved. In summary, Rayleigh stated that for incoherent illumination, two point images, of equal brightness, are just resolved when they are separated by a distance equal to the radius of the Airy disk. Rayleigh stated that optical aberrations degrade the optical 
resolution of imaging systems. Mathematically the result from diffraction theory is the following:

$$
\theta_{\text {minimum }}=\frac{1.22 \lambda}{D} \quad(\text { Rayleigh resolution criterion })
$$

where the aperture has diameter $D$, and $\lambda$ is the wavelength of the light. An optical system is denoted diffraction-limited when it can resolve two points that are separated by this angle, $\theta_{\text {minimum }}$.

The Rayleigh criterion has important limitations. It fails if the diffraction pattern does not have well defined zeros, or if they are far from the central maximum.

Sparrow's criterion is a modification of this concept, but it is not based on any assumptions about the human visual system. The Sparrow criterion of two-point resolution is the smallest distance between two points at which the minimum in the intensity distribution of the combined two luminous points vanishes. It considers incoherent point sources; two point images to be resolved if their combined intensity function has a minimum on the line between their centers. Another way to state the Sparrow criterion is that two point sources are just resolved if the second derivative, of the resulting image illuminance distribution, is zero at the point midway between the respective Gaussian-image points. A mathematical expression of the Sparrow resolution criterion is given below:

$$
\theta_{\text {minimum }}=\frac{0.95 \lambda}{D} \quad(\text { Sparrow })
$$

where the terms have the same definition in the Rayleigh resolution criterion.

Both Rayleigh's and Sparrow's classical resolution criteria assumed incoherent light. For the case of two points that emit coherent light we must combine the amplitudes of their point spread functions. The Rayleigh resolution criterion is the same as for the incoherent case. But for the Sparrow case the expression for coherent illumination is:

$$
\theta_{\text {minimum }}=\frac{1.46 \lambda}{D} \quad(\text { Sparrow-coherent case }) .
$$

It was the genius of Abbe who first extended the concepts of two-point resolution to coherent illumination for the particular case of the microscope. The Sparrow criterion has been employed with optical systems that use partially coherent light.

\subsection{The optical transfer function}

The history of the development of the optical transfer function (OTF) is critical to our understanding of the concepts of resolution. The OTF can be defined by the following ratio:

$$
\begin{aligned}
\text { OTF }= & {[\text { Fourier Transform of the light distribution in the image }] \text { divided by the } } \\
& {[\text { Fourier Transform of the light distribution in the object }] } \\
& \text { or }[\text { numerator (image) }] \text { divided by the denominator (object) }] .
\end{aligned}
$$

This relation was first written by the French physicist Pierre-Michel Duffieux (18911976) who was an assistant to Maurice Paul Auguste Charles Fabry (1867-1945). Duffieux published a series of papers starting in 1935 in which he formulated the theory of the optical transfer function. In 1946 he privately published his seminal book: L'Integral de Fourier et ses Applications à L'Optique in which he applied Fourier techniques to optics. This book had a great impact on European physicists who worked 
on optics. In 1983 John Wiley \& Sons published the second edition of Duffieux's book as an English translation under the title: The Fourier Transform and Its Applications to Optics.

Probable the most significant contribution of Duffieux was his theory of image formation that included any shape of aperture and aberration. He invoked the convolution theorem and demonstrated the Fourier transform of the function that expressed the intensity distribution in the image can be approximated by the product of the Fourier transform of the distribution in the object and the transform of a point source image. From an analysis of the fractional reduction of the Fourier components that are transmitted from the object to the image Duffieux defined the transfer function of the optical system; it depended on both the lens aperture and on optical aberrations.

The optical transfer function is a criterion for the performance of an optical system [Williams and Becklund 1989]. The definition of Williams and Becklund is quoted: "The Optical Transfer Function (OTF) is the frequency response, in terms of spatial frequency, of an optical system to sinusoidal distributions of light intensity in the object plane; the OTF is the amplitude and phase in the image plane relative to the amplitude and phase in the object plane as a function of frequency, when the system is assumed to respond linearly and to be space invariant. The OTF depends on and potentially describes the effect of diffraction by the aperture stop and the effects of the various aberrations." The latter term, aberrations, are discussed in the subsequent section of this review. The optical transfer function of the imaging system is the Fourier transform of the point spread function [Williams and Becklund 1989].

Another parameter to quantify the quality of an image is the contrast or the modulation which is defined as: $\frac{I_{\max }-I_{\min }}{I_{\max }+I_{\min }}$. The ratio of the image modulation to the object modulation at all spatial frequencies is the modulation transfer function (MTF). The MTF is defined as the modulus $|O T F|$ or the magnitude of the optical transfer function. The MTF is the magnitude response of the optical system to sinusoids of different spatial frequencies.

Harold H. Hopkins (1918-1994) is credited with the development and use of the modulation transfer function in 1962. Hopkins was a student of Duffieux at the University of Besançon. Hopkins did most of his work on Fourier optics at The Imperial College of Science, Technology and Medicine also known as Imperial College London.

\subsection{The concept of the diffraction limit}

An alternative approach to the classical criteria of resolution is based on linear system theory in which we assume that the imaging device is both linear and shift invariant [Gaskill 1978; Sheppard 2007; Van Aert et al. 2007]. We can characterize such an imaging system by its point spread function. A coherent imaging system is linear in complex amplitude and an incoherent imaging system is linear in intensity. An image formed by a linear and shift-invariant optical system will have an amplitude for the case of coherent imaging or an intensity for the case of incoherent imaging that is the convolution of the amplitude or intensity distribution of the object and the point spread function of the imaging system.

In the spatial domain the imaging system acts as a filter for spatial frequencies. The imaging system transfers each spatial frequency separately. Since the optical systems aperture is finite the transfer function is band limited. This means that above some spatial frequency the transfer function is zero. In that case, the frequencies are not transferred by the optical imaging system. From this analysis the concept of the diffraction limit emerges; it is the cutoff frequency that is denoted as the diffraction limit to optical resolution. It follows that with respect to this theory the term enhanced resolution or bandwidth extrapolation refers to techniques that reconstruct 
or recapture the frequency components that are beyond the cutoff frequency of the optical system.

The progress of novel microscopy approaches based on the precise localization of individual point emitters, such as in certain types of electron microscopy, or in Localization (Light) Microscopy (see below section 5) has made it desirable to reconsider the concept of optical resolution [van Aert et al. 2006]. In such cases, the classical resolution criteria as outlined above may no longer be appropriate if images are interpreted quantitatively instead of qualitatively. Instead, an alternative criterion may be used which relates resolution to statistical measurement precision. Van Aert et al. (2006) proposed a quantitative resolution criterion that can be used to compare the performance of coherent and incoherent imaging systems: By expressing resolution in terms of the precision with which the distance between neighboring point objects can be estimated, the proposed criterion reflects the purpose of quantitative experiments, that is, precise measurement of physical parameters. As such, it may replace Rayleigh-like classical resolution criteria that express the possibility to visually distinguish adjacent objects. Both computer simulations and experimental results have confirmed the validity of this concept (see below, section 5).

\subsection{What confounds optical resolution?}

In classical light microscopy the term resolution is related to the capability of displaying detail in an image. The term resolving power of a microscope refers to the ability to distinguish in the image two points that are in close proximity in the object. The concept of resolution is ambiguous because different authors interpret resolution in a variety of ways. What is critical to understand is that the resolution of an aberrationfree optical system will be modified by the presence of optical aberrations in the imaging system [Mahajan 2001]. This effect was shown by Karl Strehl (1864-1940) who demonstrated in his book that small aberrations in the optical system could modify the Airy disk by reducing the intensity at the maximum of the diffraction pattern and the removed light is redistributed in the outer regions of the diffraction pattern [Strehl 1894].

The resolution of an optical system will be degraded in the presence of optical aberrations. The aberrations include: chromatic aberrations, defocus, spherical, coma, astigmatism, field curvature, and distortion [Mahajan 2001]. In summary, the point spread function is equivalent to the Fraunhofer diffraction pattern of the lens aperture only for the case that the lens is free from all geometrical aberrations.

\section{What was Abbe's contribution to understanding resolution in the optical microscope?}

In the late 1800s mathematical calculations were the basis for the construction of telescopes but not microscopes which were considered too complex for analytical analysis [Masters 2008]. This situation dramatically changed when Carl Zeiss hired Abbe, a physicist with doctoral training in precise measurements. The design, testing, calibration, and construction of microscopes improved over the decades due to the mathematical foundations that Abbe developed [Masters 2007]. Abbe developed several instruments for the precision measurement of optical devices including the focimeter, the refractometer, the Abbe spectrometer, the spherometer, the thickness and height meter and the comparator. The design and construction of optical components and systems changed from "trial and error" to mathematical analysis and precision measurement. Abbe also contributed the "Abbe sine condition" which if implemented yielded optical systems without spherical aberration and without coma which resulted 
in the design and construction of aplanatic microscope objectives [Abbe 1889a,b; Volkmann 1966]. According to Born and Wolf, the sine condition was first derived by Rudolf Clausius (1822-1888) in 1864, and then by Hermann von Helmholtz in 1874 from thermodynamic considerations. In 1878 Abbe rediscovered the sine condition and realized its importance in optical design.

Among his many inventions perhaps Abbe's theory of image formation in the microscope is most relevant for the subject of this review [Czapski 1910; Masters 2008]. Abbe was puzzled that his new microscope objectives with a small angular aperture performed poorly as compared to those with a large angular aperture. Abbe's analysis of this phenomenon directed him to his theory of image formation that was based on diffraction which is described in the next section.

\subsection{Abbe's contribution to image formation in the microscope}

The motivation for Abbe's research on image formation and resolution in the optical microscope was his observation that larger diameter but less well corrected microscope objectives give more fine detail in the images than smaller diameter, but more corrected microscope objectives.

In 1873 Abbe published in German a 55 page paper with the following title: "Beiträge zur Theorie des Mikroskops und der mikroskopischen Wahrnehmung" ("A contribution to the theory of the microscope, and the nature of microscopic vision") [Abbe 1873]. This paper is unique because within this paper there is not a single diagram and not a single equation. While Abbe proposed that a more complete mathematical treatment will follow it was never published, presumably due to his health. The mathematical analysis of Albert B. Porter was published thirty two years later [Porter 1906].

Abbe analyzed the diffraction of light by the object and then the effect of the aperture. The central part of Abbe's theory is that to image objects whose dimensions are similar to the wavelength of light we cannot use the concepts of geometric optics; instead, the correct explanation of microscopic image formation requires a consideration of diffraction and interference effects.

Abbe's main assumption was that the spatial frequencies in the object to be imaged are similar to that of a diffraction grating that is illuminated by a coherent light source. The object diffracts the light into many beams, each of a different order of diffraction. The resolution is greater, by a factor of 2 , when the object is illuminated with incoherent light, as compared to coherent illumination.

A modern experimental optical set up that illustrates Abbe's concept of image formation in the optical microscope is as follows. The object is a diffraction grating that is illuminated coherently with a collimated beam of quasimonochromatic light. The object, first lens, diffraction plane, second lens, and image plane are arranged in an optical system. The first lens forms a Fraunhofer diffraction pattern of the object (a grating) in the focal plane (diffraction plane) of the first lens. In the back focal plane of the microscope objective, each Airy disk is a source that forms a spherical wave; the spherical waves interfere in the back focal plane of the objective, or the diffraction plane. This diffraction pattern is the Fourier transform of the object. The second lens is used to form an image of the diffraction pattern in the image plane. A series of masks can be used to limit the number of diffraction orders that form the diffracted image and the effect of "spatial filtering" on the resolution in the final image could be readily observed.

The role of aperture is a key concept: if the microscope objective is to form an image of an object then it must have an aperture that can transmit the entire diffraction pattern produced by the object. The more diffraction orders from the object that enters the microscope objective, the more detail that could be observed in the image. 
Abbe also astutely noted that oblique illumination increased the resolution of the microscope by a factor of 2 . The explanation was that a higher order of diffraction entered the microscope objective when the central illumination beam was shifted to one edge of the microscope objective by tilting the illumination with respect to the microscope's optical axis.

Abbe set the limit of resolution for both coherent and incoherent illumination for the optical microscope from his condition that both the central (0th order of diffraction) and at least one of the diffraction order maxima must enter the objective to achieve maximum resolution. The nondiffracted 0 th order rays and the nth order rays are separated in the back focal plane and are combined in the image plane.

For the following conditions of an object that consisted of a periodic structure (lines), for an immersion microscope objective, and a circular aperture (the microscope objective) with direct illumination, Abbe calculated this limit as $d=\frac{\lambda}{n \sin \alpha}$ for direct illumination, and $d=\frac{\lambda}{2 n \sin \alpha}$ as for the case of oblique illumination (produced by a lens of the same numerical aperture as the objective lens), where $d$ is the smallest separation that can be resolved, $\lambda$ is the wavelength of the illumination light in vacuum, $\alpha$ is one-half of the angular aperture of the microscope objective, and $n$ is the refraction index of the medium between the object and the microscope objective.

Abbe also coined the term, "numerical aperture." In microscopy, the numerical aperture, or $N A$, of a microscope objective is written as: $N A=n \sin \theta$, where $n$ is the refractive index as defined above, and $\theta$ is one-half of the angle of the cone of light that can enter the microscope objective. The angular aperture of the microscope objective is twice the angle $\theta$.

Abbe showed that it is possible to enhance the resolution of a microscope by the use of two techniques. First, the use of oblique or off-axis illumination would enhance the resolution by a factor of 2 . Second, the use of immersion microscope objectives and the use of an immersion fluid with a high refractive index would enhance the resolution by a factor approximated by the index of refraction of the fluid as compared to air.

Abbe showed that for the standard configuration of the optical microscope, under the illumination conditions that he stated, light diffraction limits the two-point resolution of an optical microscope to approximately one-half of the wavelength of the illumination light or approximately 200-300 $\mathrm{nm}$. This is known as the Abbe limit of resolution. From the analysis of Abbe on microscopic resolution it follows that in order to increase the resolution of an optical instrument it is necessary to either decrease the wavelength or to increase the numerical aperture.

Hermann Ludwig Ferdinand von Helmholtz (1821-1894) in 1874 published a paper in which he calculated the maximum resolution for an optical microscope [Helmholtz 1874]. Helmholtz used the ray tracing techniques that were often used in telescope design and he stated that the smallest separation of two distinct luminous points that could be resolved was equal to the one-half of the wavelength of illumination light. Helmholtz read Abbe's earlier contribution only after he wrote his paper, but he was able to attach a note to his paper, prior to its publication, that acknowledged the priority of Abbe. Helmholtz also in his paper acknowledged the 1803 publication by Joseph Louis Lagrange (1736-1813), Sur une loi général d'Optique in Mémoires de l'Académie de Berlin. The so called "general law of optics of Lagrange" is explained in the next paragraph. It is one of a group of invarients in optics that have numerous names. Helmholtz extended the theorem of Lagrange, which was derived for infinitely thin lenses, to apply to finite divergence-angles.

The definition of some term follows. The geometrical path length is the geometrical distance measured along a ray between any two points. The differential unit of length is:

$$
d s=\sqrt{d x^{2}+d y^{2}+d z^{2}} .
$$


The optical path length between two points $x_{1}$ and $x_{2}$ through which the ray passes is:

$$
\text { Optical path length }=\int_{x_{1}}^{x_{2}} n(x) d s .
$$

Characteristic functions in optics permit a complete description of path lengths between pairs of points in an optical system. They can be on the same side of a lens or on different sides. William Rowan Hamilton (1805-1865) was the first to consider them; they have the name Hamiltonian optics. The solution for optical ray paths involves the calculus of variations. The method is similar to that used in classical mechanics where the time integral of the Lagrangian is an extremum.

The concept of étendue and its conservation will now be explained. The étendue is known by many different names: generalized Lagrange invariant, lumininosity, lightgathering power, and area-solid-angle-product. A bundle of light rays intersects a constant $z$ plane in a small region of size $d x d y$ and has a small range of angles $d \alpha$ and $d \beta$. As the light propagates the following quantity is constant:

$$
n^{2} d x d y d \alpha d \beta=n^{2} d A d \alpha d \beta=n^{2} d A \cos \theta d \omega
$$

Where $d A=d x d y$ is the differential area, $d \omega$ is the solid angle, and $\theta$ is measured relative to the normal in the plane. The integral of this last equation is called the étendue and is conserved.

$$
\int n^{2} d x d y d \alpha d \beta=\int n^{2} d A d \alpha d \beta=\int n^{2} d A \cos \theta d \omega .
$$

In his paper of 1896, on image formation in the microscope in which he first analyzed the aperture and then the object [Rayleigh 1896], Rayleigh reached similar conclusions as did Abbe; and he stated explicitly that the maximum resolution achievable was $\lambda / n$ ( $\lambda$ vacuum wavelength, $n$ refraction index).

In Abbe's theory, he first considered the diffraction by the object, and then he considered the effect of the aperture of the microscope objective. Rayleigh used Lagrange's general law of optics [see the previous paragraph], known as the generalized Lagrange invariant, and Fourier analysis to calculate the diffraction pattern of apertures with various shapes, as well as the diffraction pattern from gratings. According to Born and Wolf, both theories are equivalent.

Rayleigh then states that the two-point resolution in an optical microscope can only be improved by reducing the wavelength of light (he suggests photography, perhaps with ultraviolet light) or by affecting the numerical aperture by increasing the refractive index of the medium in which the object is situated.

August Karl Johann Valentin Köhler (1866-1948) has published a modern analysis of Abbe's theory of image formation in the microscope that is based on the complex Fourier transform. The first part of his paper presents a historical review of the publications from 1873 to 1910 which is highly instructive [Köhler 1981]. Several other historical sources can be consulted to provide additional material on the contributions of Abbe to the development of the optical microscope [Abbe 1873, 1889a,b; Lummer and Reiche 1910; Masters 2007].

In summary, Abbe in his theory of the microscope showed how diffraction contributes to image formation in the optical microscope. In Abbe's theory the interference between the 0th-order and higher-order diffracted rays in the image plane forms image contrast and determines the spatial resolution. At least two different orders of diffracted light must enter the lens for interference to occur in the image plane.

Abbe showed the role of the numerical aperture $(N A)$ and the wavelength of the light in the resolution of the microscope. He proposed that the optical resolution of the 
light microscope could be improved by either decreasing the wavelength of the light or by increasing the numerical aperture $(N A)$ of the microscope. Finally, he conceived and demonstrated his theory with a series of simple diffraction experiments that involved masks and apertures in the focal plane which altered the spatial frequencies that formed the image. These clever visual experiments helped to convince others of the correctness of his theory. While he promised to publish a detailed paper on the mathematical development of his theory he died before this occurred.

\subsection{How Porter used physical optics to explain Abbe's diffraction theory}

In 1876, three years after Abbe published his theory of image formation in the microscope, Abbe traveled to London to demonstrate his diffraction theory with a series of experiments which he demonstrated in front of the Royal Microscopical Society. With his set of gratings, apertures and a microscope Abbe gave public demonstrations of his theory.

As stated above, Abbe's promised mathematical paper of his theory of image formation in the microscope was never published. Porter's mathematical paper described Abbe's theory and Abbe's experiments in only 12 pages because he used Fourier analysis to present the mathematical foundation of Abbe's theory. The basic concepts of Abbe's theory of image formation in the microscope were elegantly illustrated in the experiments of Porter that are based on the early experiments performed by Abbe and which he used in his London demonstrations [Porter 1906].

Porter noted that if the object is a transmission grating with alternate opaque and transparent lines, then Fourier's theorem can be applied to this problem. When the objective lens forms a real image of the grating the harmonic components of the diffracted light are combined in the focal plane. As the aperture of the objective lens is widened, higher and higher orders of the objects diffraction pattern can enter the lens, the result is a sharper image that more closely resembles the object. Porter also showed that the resolving power of the microscope could be increased by using light of a shorter wavelength, i.e. violet or ultraviolet light. In Porter's microscope experiments normal working conditions were used: central illumination, and circular diaphragms centered on the optical axis.

In Porter's experiments that were modeled after those devised by Abbe three decades earlier, an object was a fine wire mesh that is illuminated by collimated, coherent light. In the back focal plane of the imaging lens (the microscope objective) the Fourier spectrum of the object is located, and in the image plane the various Fourier components are recombined to form the image for the wire mesh object. Porter then showed that various masks could be placed in the focal plane (an iris, a slit or a small stop), and thus it is possible to manipulate the spatial frequency spectrum of the image.

\section{Early optical techniques to enhance microscopic resolution}

The history of the microscope is replete with developments which enhance the resolution and the contrast of the image and decrease the aberrations of the optical system [Masters 2008; 2009a]. The early microscopes operated in the far-field and Abbe's and Rayleigh's analyses of image formation in the microscope indicated that to increase the two-point resolution it is necessary to decrease the wavelength of the light or to increase the numerical aperture. Abbe showed that even with a high refractive index fluid between the specimen and the objective, the upper magnitude of the numerical aperture was limited in the standard microscope design to the value of the refractive 
index $n$ (i.e. a practically useful maximum around 1.5); thus, researchers attempted to reduce the wavelength of the light. Abbe's demonstration experiments on imaging fine ruled gratings showed the effect of shorter wavelength light (blue versus red light); the blue light increased the two-point resolution. That demonstration stimulated the development of an ultraviolet microscope. Following the work of de Broglie, who showed that particles can be characterized by their wavelength, researchers began to explore the use of electrons and electric and magnetic lenses to focus electron beams as the light sources for new forms of microscopes: electron microscopes [Masters 2009b]. The wavelength of electrons $(1-2 \AA)$ in beams is much smaller than the wavelength of ultraviolet light and thus the resolution could be vastly improved as compared to visible light.

\subsection{Techniques to decrease the wavelength of illumination}

\section{Ultraviolet microscope}

The motivation to develop an ultraviolet microscope followed from Abbe's theory of image formation in the microscope. It was predicted [Rayleigh 1896] that the shorter wavelengths of the ultraviolet light, as compared to visible light, would enhance the resolution of the microscope.

In 1904, August Köhler, working in the Jena Zeiss factory, invented an ultraviolet microscope that preceded the fluorescence microscope. A camera was required to detect the very weak image. The microscope used a quartz monochromatic ultraviolet microscope objective that was previously developed by Moritz von Rohr. Köhler noted that the ultraviolet light excitation on a crystal of barium platinum cyanide resulted in fluorescence in the visible spectrum. Köhler made some early prescient observations with his ultraviolet microscope: the observation of autofluorescence of biological specimens that were excited with ultraviolet light, and the observation of the ultraviolet image of unstained chromatin in the cell nucleus with incident light of $275 \mathrm{~nm}$ [Masters 2008].

\section{Electron-microscopes}

The early development of the electron microscope depended on a long series of advances in electron optics [Masters 2009b]. The practical development of the electron microscope depended on both an understanding of electron optics and on correcting the aberrations that were caused by the magnetic lenses. In 1931, Max Knoll (18971969) and Ernst August Friedrich Ruska (1906-1988), working in Berlin constructed a two-stage transmission electron microscope with magnetic lenses. A wire mesh was used as the object. In 1931, Ruska improved their transmission electron microscope and demonstrated its capability to surpass the resolving power of the light microscope [Ruska 1979; 1986]. The enhanced resolution of the electron microscope has resulted in seminal advances in cell biology, neuroscience, virology, and material science [Masters $2009 \mathrm{~b}]$.

\section{X-ray microscopes}

Another approach to realize enhanced resolution with wavelengths much shorter than possible in ultraviolet light microscopy has been to use soft X-ray and synchrotron sources. For contrast, absorption differences (carbon, oxygen) or phase contrast are 
applied. For focusing the imaging X-rays, various techniques may be used, especially appropriately manufactured Fresnel plates.

Approaches to construct X-ray microscopes date back already to the 1930s and since then have undergone substantial improvements [Newberry et al. 1959; Schmahl et al. 1989, Dierolf et al. 2010]. With soft X-rays, it has become possible to examine entire cells with a three-dimensional (3D) resolution down to few tens of $\mathrm{nm}$ [Chao et al. 2005; Schneider et al. 2010].

\subsection{Slit illumination: the ultra-microscope to detect colloids}

A point source of light will be imaged at the resolution of the optical microscope. A single luminous particle whose lateral dimension is less than the resolution of the microscope will also be imaged at the resolution of the microscope. For example, the fluorescent images of the cytoskeleton show filaments whose cross-sectional dimensions (a few tens of $\mathrm{nm}$ ) are far below the resolution of the standard wide-field fluorescent microscope; they form an image at the resolution of the microscope. Alternatively, it is possible to detect with a conventional microscope a luminous particle whose lateral dimensions are far below the resolution of the optical microscope. This is illustrated in the description of the ultramicroscope which played a major role in the field of colloid chemistry. However, since the resolution power remained the same as in conventional microscopy, it was not possible to analyze ("image") structural details in such particles.

In 1903 Henry Friedrich Wilhelm Siedentopf (1872-1940), who worked at the optical works of Carl Zeiss, collaborated with Richard Adolf Zsigmondy (1865-1929) and they invented the ultramicroscope to observe (detect but not "image", see above) colloids. Their ultramicroscope could detect each single colloid particle as a bright spot of light and thus localize them, i.e. assign them a position relative to microscope system coordinates. However, for particles much smaller than the wavelength used, this spot size (the Airy disk) is practically dependent only on the microscope system and the wavelength applied, and hence does not confer information on the size of the object.

In the Siedentopf-Zsigmondy microscope the illumination is perpendicular to the optical axis of the microscope. They used the dark-field illumination technique in which each colloid particle is detected by its scattered light that enters the microscope objective and is seen by the eye of the observer as a point of light. The effect is similar to how we observe particles of dust in a sunbeam. At the time it was not accepted that it would be possible to observe (detect) a particle that is much smaller than the resolution of the optical microscope.

Siedentopf constructed a dark-field condenser that blocked the incident illumination from entering the microscope objective and that improved the contrast. He reported that the use of ultraviolet light for the illumination produced specimen fluorescence, and that fluorescence was a problem since it reduced the contrast in the microscope. In 1914 Siedentopf and Zsigmondy further developed their original invention and produced a slit ultramicroscope and an immersion ultramicroscope. Zsigmondy received the Nobel Prize in Chemistry for the year 1925 for his work on colloid chemistry (Zsigmondy 1907; Zsigmondy 1909). It is of interest that the modern microscopic techniques that are based on "illumination with a light sheet" [Keller et al. 2007, 2008; Greger et al. 2011] follow from the early works of Siedentopf and Zsigmondy on lateral illumination, in combination with the experience gathered during the 1990s with laser excitation and orthogonal detection schemes (Lindek et al. 1994, 1996). 


\subsection{Erwin Müller: Field-ion microscope to image atoms}

The field emission microscope, invented by Erwin Wilhelm Müller (1911-1977) in 1936, provided a resolution superior to the standard light microscope [Müller and Tsong 1969]. Its development began with Müller's early investigations on field emission in the 1930's when he worked in Berlin at Berlin-Siemensstadt [Müller 1937]. He suggested that this new type of microscope could be used to study the properties of metal surfaces and adsorbed layers.

The basic design of the field emission microscope is as follows. A specimen in the form of a needle is maintained under a very high vacuum and the tip is subjected to a very large negative voltage. The electric field at the tip is of the order of $10 \mathrm{~V}$ per nm. This strong electric field causes electrons to be emitted for the tip by quantum mechanical tunneling. The emitted electrons are accelerated towards the screen containing phosphors, and they form a magnified image of the atomic arrangement on the surface of the needle. The lateral resolution is of the order of $20 \AA$. Individual atoms could not be observed.

Several years later in 1951 Müller, now working at the Fritz Haber Institute in Berlin, developed the field-ion microscope, in which gas atoms replaced the electrons of the earlier field emission microscope, and he achieved atomic resolution with the ability to image individual atoms on the surface of the needle. In his new microscope he made the specimen of positive polarity and used a gas such as hydrogen, helium or neon, to enter the apparatus. The atoms of the gas are ionized a few angstroms above the atomic surface, and the helium or neon ions are accelerated to the phosphor imaging screen to form the image of the atoms in the lattice of the specimen. The spatial resolution of the microscope is about $2 \AA$.

In 1963 Müller could write "Field ion microscopy is the only means known today for viewing directly the atomic structure on solid surfaces in atomic detail" [Müller and Tsong 1969]. It was the first microscope to achieve atomic resolution. The prescient developments of Müller and his colleagues formed the basis of much of the subsequent work in scanning probe microscopes (SPM) that achieved atomic resolution; these microscopes will be discussed in a subsequent section (4.6).

\subsection{Confocal microscopes: to enhance the 3D resolution}

A standard wide-field microscope that images a volume of the specimen does not have any "optical sectioning" capability, or the capability to discriminate against light that comes from regions that are not contained in the focal place of the microscope objective. A plot of intensity of the image versus distance from the focal plane is a horizontal line.

Since the 1960s, novel optical ideas were conceived and realized to overcome this severe limitation of light microscopy in the study of three dimensional structures in the biosciences. To these developments of 'confocal laser scanning fluorescence microscopy' (CLSM) and reflected light confocal microscopy, many scientists and engineers have contributed in a process of several decades of duration, either by theoretical concepts, or by the experimental realization and the introduction of these novel approaches into the biosciences [Masters 1996]. CLSM made possible for the first time to create sharp three-dimensional images of a variety of biological structures, combined with a highly superior contrast. Presently, the typical resolution of a CLSM (in terms of FWHM) is $200 \mathrm{~nm}$ in the object plane $(x, y)$ and $600 \mathrm{~nm}$ along the optical axis, i.e. still within the limits of conventional theories of resolution. Due to the many and excellent reports existing on the history of confocal microscopy and due to the focus of this present report to enhanced resolution techniques beyond these values, we have to restrict ourselves to a few remarks. 
A confocal microscope is a scanning microscope where the image is formed by 'point-by-point' excitation of the object and 'point-by-point' registration of the optical response [Masters 1996]. The spatial filtering may be obtained via a set of conjugate apertures or slits (one for illumination, and one for detection) which results in an "optical sectioning" capability. The out-of-focus light is rejected by a pinhole located in front of the detector. A plot of intensity versus distance from the focal plane decreases with distance from the focal plane. In the case of laser illumination with appropriately shaped Gaussian beams, only the detection pinhole but not the illumination pinhole was necessary [Davidovits and Egger 1971].

While a confocal microscope as compared to a standard wide-field microscope has a slight enhancement in the axial and the lateral resolution (the optimal lateral improvement is 1.4), the main reason for its utility is the enhanced axial resolution which permits "optical sectioning" of specimens and enhanced contrast in the resulting images [Masters 2010]. A standard wide-field microscope has the contrast severely degraded by light from outside the focal plane of the microscope objective which contributes to the image. In the 1980s, John Sedat and David Agard introduced threedimensional deconvolution techniques for biological light microscopy which allowed to improve the contrast of such biological images considerably [Agard and Sedat 1983]. The removal of out-of-focus information made possible by such methods was based on the mathematical combination of a series of wide field images taken from the same object at different focal positions.

Masters has edited a book that includes the key historical papers and the patents related to confocal imaging systems and demonstrated the linkages between the many inventions and reinventions [Masters 1996]. This volume, and its selected papers and patents that are reprinted in full, contains examples of history that are not commonly known.

For example, the invention of the confocal microscope is often attributed to Marvin Lee Minsky who filed his patent on November 7, 1957. In fact, Minsky's invention was partly predated by the paper that Hiroto Naora published in Science (vol. 114, 279280, September 14, 1951). Furthermore, it is often not recognized that a practical use of the confocal principle in fluorescence and reflected light microscopy became possible only after the development of laser sources with appropriately short wavelengths in the $1960 \mathrm{~s}$.

Another misconception is the invention of the scanning mirror confocal microscope that is often attributed to G. J. Brakenhoff [Brakenhoff et al. 1979, 1985]. In fact, G. M. Svishchev invented a scanning mirror confocal microscope to image the brain and published it in Optics and Spectroscopy (vol. 26, 171-172) in 1969.

\subsection{Near-field microscopes}

Near-field and far-field optics are different and that difference is exploited in the nearfield microscopes. Subwavelength resolution and imaging are achieved by coupling the light from the specimen to an optical element that is located a subwavelength distance away. When the detector is less than one wavelength away from the specimen, the resolution is given by the size of the aperture and not the wavelength of the illumination [Courjon 2003; Paesler and Moyer 1996]. With standard light microscopes (using a fixed wavelength of constant intensity) far-field diffraction sets a limit to the spatial resolution (the Abbe limit of resolution).

Near-field microscopy presents another example of a prescient invention that years later resurfaced and then various aspects of the original invention were rediscovered [Jutamulia 2002]. In 1928 Edward Hutchinson Synge, first in a correspondence 
with Albert Einstein and shortly afterwards in a publication described his invention of the microscope that today we call the near-field scanning optical microscope [McMullan 1990; Synge 1928]. Synge's proposed microscope had a light source behind an opaque metal film that had a small orifice of about $100 \mathrm{~nm}$. The orifice was situated within $100 \mathrm{~nm}$ of the surface of the specimen, and the image was formed by point or raster scanning the small orifice over the specimen.

In 1944, the physicist Hans Albrecht Bethe (1906-2005) published a seminal paper on the diffraction of electromagnetic radiation by a hole whose diameter is small compared to the wavelength of the illumination [Bethe 1944]. Bethe assumed that the small hole is in an infinite plane conducting screen that separates two cavities. It follows that one can assume that the electromagnetic field is almost constant over the hole. First, Bethe shows that the standard formulation of Kirchhoff's diffraction theory is not valid since the Kirchhoff solution does not satisfy the boundary conditions of the problem. Then he proceeds to provide a correct mathematical solution to the problem which does satisfy the boundary conditions.

Eric A. Ash and G. Nicholls stimulated by the work of Lukosz who showed how to enhance the resolution of an optical microscope by a factor of two discarded the attempt to achieve higher orders of enhancement of resolution by wide-field illumination since the diffraction-limited stop at the focus of a lens is of the order of one wavelength. Instead they proposed fabricating an aperture, whose diameter is much smaller than the wavelength of the illumination radiation, and scanned the aperture over the surface of the specimen with a distance that was much smaller than the wavelength of the illumination radiation (Ash and Nicholls 1972). Their proof of principle experiments were conducted at a frequency of $10 \mathrm{GHz}(\lambda=3 \mathrm{~cm})$. They clearly imaged a grating with a line width of $\lambda / 60$. They end their prescient paper with the suggestion to build an enhanced resolution optical microscope that would operate in the visible region.

It took until 1982 for Winfried Denk who was working in Dieter W. Pohl's IBM Research Laboratory in Rüschlikon, Switzerland to demonstrate near-field imaging in the visible wavelength region. The authors demonstrated subwavelength resolution optical image recording by moving an extremely narrow aperture along a test object equipped with fine line structures. Details of $25 \mathrm{~nm}$ size can be recognized using $488 \mathrm{~nm}$ radiation [Pohl et al. 1984]. In the same year at Cornell University, A. Lewis, Isaacson, Harootunian, and Murray published a paper which described their development of a $500 \AA$ spatial resolution light microscope [Lewis et al. 1984; Betzig et al. 1986].

\subsection{Scanning probe microscopes}

Scanning probe microscope (SPM) have been developed in many versions, but the basis is scanning a small probe over the surface of the specimen, and the image is generated point-by-point, through the interaction of the probe tip and the local region of the specimen [Martin 1995].

The invention of the scanning tunneling microscope (STM) is credited to Gerd Binnig and Heinrich Rohrer who were working at IBM in Research Laboratory in Rüschlikon, Switzerland in 1982 [Binnig and Rohrer 1982]. For their seminal work they received the Nobel Prize in 1986. STM requires that the specimen be electrically conducting. The interaction between the specimen and the probe tip is the tunneling of electrons between the tip and the specimen. The tunneling current is extremely sensitive to the specimen height; the tunneling current decreases by an order of magnitude for each $1 \AA$ increase in tip to sample distance. SPM can achieve a $0.1 \mathrm{~nm}$ lateral resolution and $0.01 \mathrm{~nm}$ depth resolution which permits the imaging and the manipulation of single atoms. 
In 1986, Binnig, Quate, and Gerber invented the atomic force microscope (AFM) which is one of the most popular implementations of SPM. The physical interaction in the AFM is the force between the tip and specimen; it is mediated by van der Waals forces, electrostatic forces, magnetic forces and Pauli repulsive forces.

A recent Science paper demonstrated the ability of atomic force microscopy to not only resolve single atoms within a molecule on a surface, but to identify the chemical structure of the atom; this study demonstrates the complete structure of a molecule with atomic resolution [Gross et al. 2009]. These results were obtained at atomic resolution by probing the short-range chemical forces with the use of noncontact AFM.

\subsection{Microscopes based on the optics of metamaterials}

Recent developments in the development of metamaterials have demonstrated alternative techniques to image below the diffraction limit. Metamaterials are engineered materials that contain microscopic inhomogeneities that are responsible for their macroscopic physical properties. In 1968 the Russian physicist Victor Georgievich Veselago proposed that metamaterials could be engineered to yield a negative refractive index. He suggested that negative refraction can occur if both the electric permittivity and the magnetic permeability of a material are negative. Furthermore, a plane metamaterial could act as a lens (a point object is imaged as a point image) and have a negative refraction [Veselago 1968]. This was first published in Russian in 1967, and the English translation was published in 1968.

Recent theory has predicted a superlens that could produce subdiffraction-limited images in the near-field [Pendry 2000]. The metamaterial would compensate for wave decay and use both the propagating and the evanescent fields to form the image; both contribute to the resolution of the image. As John Pendry has shown in his theory when an object is situated in front of a material with a refractive index, $n=-1$, the near field light, which usually decays exponentially with distance from the interface, is focused within the material, and it is also focused outside of the lens [Pendry 2000]. He suggested the use of silver and in 2005 his earlier suggestion was experimentally validated [Fang et al. 2005].

Pendry gave the example of a medium in which both the dielectric function, $\varepsilon$, and the magnetic permeability $\mu$, are both negative. A slab of this medium bends the light to a negative angle at the normal to the surface, the light from the object point that was diverging, converges to a point within the medium. As the light exits the medium, it is refocused for a second time.

The anomalous refraction of light by negative index materials can be explained as follows. The causality principle and conservation of tangential wave vectors of the electromagnetic field require that if the light is incident from a positive-index material to a negative-index one, the refracted light lies on the same side as the incident light with respect to the surface normal [Liu and Zhang 2011].

In a recent experimental advance that used silver as an optical superlens, the authors demonstrated sub-diffraction-limited imaging with 60-nanometer half-pitch resolution, or one-sixth of the illumination wavelength [Fang et al. 2005]. The superlens operated in the near-field with a magnification equal to 1. As explained by Fang et al. "... a superlens is predicted to substantially enhance evanescent waves, compensating for the evanescent loss outside the superlens and thus restoring an image below the diffraction limit" [Fang et al. 2005].

In 2007 a superlens was devised that could be integrated into a far-field microscope and had a resolution of $70 \mathrm{~nm}$ or $\lambda / 7$, which is below the diffraction limit for visible light [Smolyaninov et al. 2007]. Their superlens consisted of a multilayer metamaterial of alternating layers of positive and negative refractive index. Our understanding of 
the fabrication of new metamaterials, their physical properties, and their applications in optics, in particular enhanced resolution in the visual wavelengths is the subject of a recent critical review [Liu and Zhang 2011]. To what extent this type of resolution enhancement can be applied also using long working distances (like in conventional far field microscopy), still appears to be controversial. However, even so such techniques should be highly valuable to complement scanning near field optical techniques to analyze surface structures.

\section{Modern techniques to enhance the resolution of far-field optical microscopy}

With the advent of laser technology in combination with novel optical and photophysical approaches, highly sensitive and fast detection systems and computer based evaluation procedures, new developments made it possible to radically overcome the conventional light-optical diffraction limit of $\sim 200 \mathrm{~nm}$ laterally and $600 \mathrm{~nm}$ axially also in far-field light microscopy (i.e. for distances between emitter and first lens of the optical system larger than several hundred wavelengths). Since the 1980s, a number of far-field based "super-resolution" light microscopy (or "nanoscopy") techniques have been developed to allow an optical resolution far beyond that threshold. Of particular interest to the life sciences is the possibility to achieve such a resolution enhancement also in the physiological temperature range $(\sim 300 \mathrm{~K})$ and in live cells.

Presently, three main "nanoscopy" families for resolution enhancement in farfield fluorescence microscopy have been established: "nanoscopy" based on highly focused laser beams, such as 4Pi-, STED (STimulated Emission Depletion), and GSD (Ground State Depletion; using focused excitation in the original publication) microscopy; nanoscopy based on Structured Illumination Excitation (SIE), such as Standing Wave (SW), Spatially Modulated Illumination (SMI), Structured Illumination (SI) and Patterned Excitation Microscopy (PEM); and nanoscopy allowing enhanced resolution even in the case of homogeneous excitation made possible by a variety of approaches which may be summarized under the names of localization microscopy and Superresolution Optical Fluctuation Imaging (SOFI). In particular, since the mid-1990s various types of localization microscopy approaches have been developed. With such techniques, it has become possible to analyze the spatial distribution of fluorescent molecules with a greatly enhanced resolution down to a few nanometers. Presently, nanoscale images can be obtained in the far field that were previously only obtained with X-ray/electron microscopy or with near field techniques. With regard to some of these novel enhanced resolution techniques, it may be noted that for one of the authors (CC) this is a participant's history, which may imply involuntary biases, for which he offers an apology.

\subsection{Focused nanoscopy}

\section{Enhancement of resolution by increasing the aperture angle}

For point-like illumination and detection as in confocal laser scanning fluorescence microscopy, the resolution depends on the volume of the illumination PSF; the smaller this volume, the better the resolution; the larger the aperture angle, the smaller the PSF volume [Hell and Stelzer 1992a]. For technical reasons, however, the maximum aperture of an oil immersion lens is limited to an aperture angle of about $140^{\circ}$. In the following section, we first describe ideas and quantitative concepts to increase the aperture angle and thus to realize for the first time a substantially enhanced 3D resolution. 


\section{The $4 \pi$-microscopy concept}

First ideas to overcome the conventional resolution limit as defined above (called the "Abbe-limit") for optical resolution using a confocal laser scanning fluorescence microscopy approach with an extension of the sterical aperture angle $(2 \alpha)$ beyond $2 \pi$ were proposed in the 1970s [Cremer and Cremer 1978].

This early laser scanning " $4 \pi$ "-microscopy concept was based on experimental experience with diffraction limited focusing of coherent light in the submicrometer range and fluorescence excitation [Cremer et al. 1974]. The object is illuminated "point-bypoint' by a focused laser beam using a " $4 \pi$-geometry"; i.e. instead of focusing the light through a single high $N A$ objective lens $\left(\sin \alpha_{\max }=\sin 90^{\circ}=1,2 \alpha_{\max }=180^{\circ}\right.$ corresponding to a sterical aperture angle $2 \pi$ ), the coherent light is focused from all sides (sterical angle $4 \pi$ ) by constructive interference [Cremer and Cremer 1972]. The fluorescence optical signal emitted from each object point (using point-by-point scanning, in this case high precision stage scanning) is registered via a detection pinhole in the image plane which excludes contributions outside the central maximum of the diffraction pattern produced in the image plane by a point source. The individual signals obtained from object sites $(x, y, z)$ are then used to electronically construct an image with improved optical resolution by assigning the signals to sites $(x, y, z)$. It was suggested that with $4 \pi$-focusing ( "... the excitation light is incident from all sterical directions..."), the focal diameter could be reduced to a minimum below the Abbe limit of $\sim 200 \mathrm{~nm}$ at least in one direction [Cremer and Cremer 1978]. To realize a full $4 \pi$-geometry, it was proposed to generate a " $4 \pi$ point hologram," either experimentally from a source with a diameter much below the wavelength of the light emitted from such a source [Cremer and Cremer 1972], or to produce it synthetically on the basis of calculations [Cremer and Cremer 1978]. This " $4 \pi$ hologram" should replace a (single) conventional lens (maximum numerical aperture $N A \sim 1.5$ ) which allows focusing only down to about the conventional resolution limit of $\sim \lambda_{\text {exc }} / 2$, where $\lambda_{\text {exc }}$ the vacuum excitation wavelength used (Rayleigh 1896). In addition to resolution enhancement to values below $\lambda_{\text {exc }} / 2$, it was envisaged that such a hologram based $4 \pi$-microscopy should allow a much larger working distance than achievable by lens based high resolution systems. The authors stated "Whether it might be possible to produce point-holograms in which a larger spherical angle than approximately $2 \pi$ is used for focusing, and whether such holograms might be really used in a laserscanning-microscope, remains to be investigated. Perhaps the use of non-plane pointholograms might result in a better focusing even if the spherical angle is considerably smaller than $4 \pi$ "

In the original " $4 \pi$ " concept [Cremer and Cremer 1978; for first speculative ideas see Cremer and Cremer 1972], a 'hologram' was generally defined as a device to produce the boundary conditions which together with appropriate illumination and refraction index conditions yields the reconstructed waves, leaving open questions such as production, material problems, or direction, amplitudes and coherence of the incident and the reconstructed waves. Furthermore, it was assumed that the amplitudes and the incident angles of the coherent waves illuminating the $4 \pi$-point-hologram can be varied almost independently of each other; i.e. the concept was not limited to the classical assumptions [Born and Wolf 1980] used to calculate focal intensities. Furthermore, the use of (appropriately combined) plane point-holograms for focusing the exciting beam in the laser scanning microscope was suggested to be advantageous at least with respect to the much larger working distance available.

In the first, speculative considerations on the possibility of enhanced resolution by $4 \pi$-microscopy [Cremer and Cremer 1972], the optical signals derived by the scanning $4 \pi$ beam were not further specified. However, it was proposed that they might include signals from one or more types of organic molecules (e.g. a protein) with 
two photoinducible and optically distinguishable conformations A, B. According to the authors, these conformations might be changed by appropriate laser frequencies/intensities from A to B or B to A, either reversibly or irreversibly. Accordingly, it was proposed that at appropriate conditions, the optical signals derived from A or B could be discriminated. However, it was assumed that the spatial positioning accuracy corresponded to the size of the $4 \pi$-focus, i.e. the possibility to achieve a localization accuracy better than the FWHM of the $4 \pi$-PSF was not considered. In a later publication [Cremer and Cremer 1978], the optical interaction envisaged was specified to be fluorescent molecules.

In the special case that for fluorescence excitation a hypothetical continuous wave, monochromatic spherical wavefront of constant intensity is focused in a full $4 \pi$ geometry, theoretically in the far field where the distance $r$ from the focusing device $[2 \pi / \lambda] r \gg 2$ [Chen 2009]), the focal diameter achievable in such a $4 \pi$-geometry may be estimated: assuming a vacuum wavelength $\lambda_{\text {exc }}=488 \mathrm{~nm}$ and a refraction index of $n=1.5$, and assuming a limited focal diameter of about one-third of the excitation wavelength [Hell 2007], this would result in a focal diameter:

$\emptyset_{\mathrm{ill4 \pi}}=0.33 * \lambda_{\text {exc }} / n=0.22 \lambda_{\text {exc }}=107 \mathrm{~nm}$, i.e. considerably smaller than the typical minimum spot size (Full-Width-at-Half-Maximum, FWHM) for fluorescence of $\emptyset_{\text {ill }}=0.5 \lambda_{\text {exc }} / N A \sim 0.5 \lambda_{\text {exc }} / 1.4=0.36 \lambda_{\text {exc }}=175 \mathrm{~nm}$.

Recently, results of electromagnetic numerical calculations have been reported [Cremer et al. 2010] which were based on the assumption of numerous coherent light sources evenly distributed on a closed enveloping surface corresponding (with respect to the sterical angle covered) to a sterical angle of $4 \pi$; these calculations confirmed the possibility to focus in such a " $4 \pi$ geometry" monochromatic coherent light of constant intensity to an isotropic focus with a halfwidth (FWHM) around $0.2 \lambda_{\text {exc }}$.

Concerning the limits of $4 \pi$ focusing (and hence of resolution), the authors greatly overestimated its potential for resolution enhancement. In spite of this erroneous overestimation of the limits of holographic reconstruction given by optical wave theory, the idea to obtain substantially enhanced resolution by point-by-point scanning of a constructively focusing laser beam using a $4 \pi$ geometry (put forward by the authors as a 'hypothesis' to be discussed) appears to have been the first published example of considerations to realize a substantial resolution enhancement by some type of "point spread function engineering" based focused nanoscopy. However, so far the concept to achieve resolution enhancement by using point holograms in a $4 \pi$ geometry (e.g. by a combination of numerous point holograms arranged as a polyhedron) has not been realized experimentally.

\section{Pi-Microscopy}

At the beginning of the 1990s, the 4Pi-confocal fluorescence microscopy concept was first described in a patent application by Stefan Hell [Hell 1990]. Stefan Hell had just obtained his Ph.D. in Physics on a topic of confocal imaging [Hell 1990a] at the Institute of Applied Physics (presently Kirchhoff-Institute of Physics) at Heidelberg University, where also the C. Cremer group was located since 1983 when CC was appointed there as Professor of Applied Optics and Information Processing; in the following years, Stefan Hell's 4Pi-microscopy concept was further developed and experimentally realized [Hell 1990, 1992; Hell and Stelzer 1992a,b; Hell et al. 1994a; Hänninen et al. 1995]. Initially this early work on 4Pi microscopy was performed at Heidelberg/Germany, where it had been supported by the laboratories of Ernst Stelzer (Advanced Light Microscopy, European Molecular Biology Laboratory) and of C. Cremer (Applied Physics/Kirchhoff Institute, University Heidelberg), resulting in a number of joint early publications [Hell and Stelzer 1992; Hell et al. 1994a,b; Lindek et al. 1994]. It may be noted that the $4 \pi$-microscopy paper of 


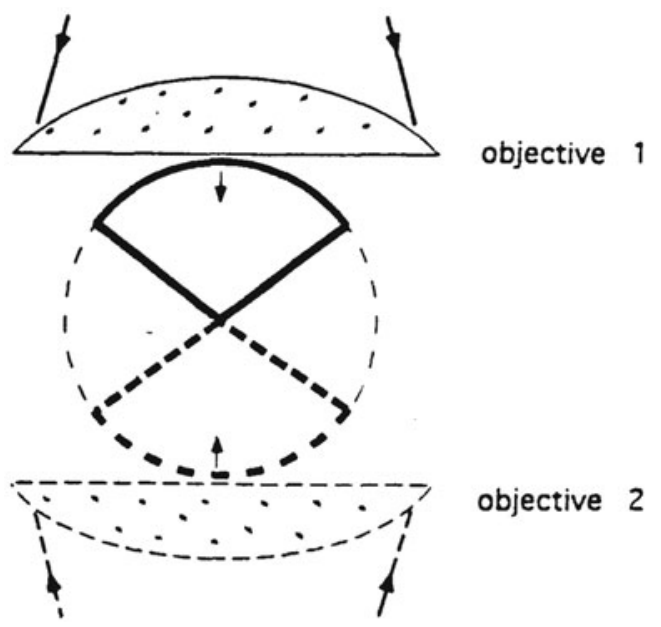

Fig. 2. Scheme of illumination in confocal laser scanning 4Pi-microscopy [Hell et al. 1994a]. Ideally the laser light is focused from all sides; i.e. the a full sterical angle $(4 \pi)$ is used, as indicated by the thin broken line (----). As an approximation to a full $4 \pi$ angle, in the 4Pi-microscopy approach two opposing high numerical aperture objective lenses are used for constructive focusing. This limits the sterical angle available for constructive focusing to the sum of the aperture angle of the objective lens 1 (above), as indicated by the bold continuous line $(-$ ) and of the aperture angle of the objective lens 2 (below), as indicated by the bold broken line (----). Reprinted with permission from Hell, S.W. et al. 1994. Appl. Phys. Lett. 64: 1335. Copyright 1994 American Institute of Physics.

[Cremer and Cremer 1978] has been quoted already in one of the very first publications of Hell and Stelzer (ref. 4 in [Hell and Stelzer 1992b]).

Hell and Stelzer started from the basic idea of confocal laser scanning fluorescence microscopy [CLSM]. They stated that "the volume of the point-spread function depends on the numerical aperture of the microscope objective and on the wavelengths of the emission and the excitation light. The smaller this volume the better the resolution of the microscope" [Hell and Stelzer 1992b]. They observed that a substantial decrease of this volume and hence a substantial enhancement of the 3D resolution of the CLSM should become possible by a uniformly spherical illumination.

However, in contrast to the holographic $4 \pi$ microscopy concept [Cremer and Cremer 1978], "the basic idea of the 4Pi confocal fluorescence microscope is to use two microscope objectives with a common focus to increase the aperture of the microscope. An increase of the aperture along the axial direction is achieved either when the objectives are illuminated with coherent wave fronts that are interfering constructively in the common focus or when the fluorescent light that is collected from both sides interferes constructively in the common point detector. Since a solid angle of $4 \pi$ is not achievable, the term $4 \mathrm{Pi}$ was chosen to indicate the basic idea with a simple and straightforward term." [Hell and Stelzer 1992a, p. 2160].

Consequently, while the $4 \pi$-confocal fluorescence microscopy idea of [Cremer and Cremer 1978] was based on constructive lens-free focusing from all sides in a full sterical angle $4 \pi$ (due to the larger working distances, the lens-free holographic arrangement should have allowed $4 \pi$ focusing, in contrast to the sterical hindrance in focusing light through more than two highest $N A$ oil immersion objective lenses), the 4Pi-microscopy approach [Hell 1990b; Hell and Stelzer 1992a,b] was based on increasing the sterical aperture angle beyond $2 \pi$ by two opposing conventional lenses of high numerical aperture. In this way, the maximum aperture angle was increased from $2 \times 68^{\circ}=136^{\circ}($ oil immersion lens with $N A=1.4)$ to $2 \times 136^{\circ}=272^{\circ}$ (Figure 2 ) 
Furthermore, for the first time rigorous electromagnetic numerical calculations of the focusing and the confocal $4 \mathrm{Pi}$ point spread function using two opposing objective lenses of high numerical aperture were performed [Hell and Stelzer 1992a]. For this, a normalized electric field of linearly polarized light was assumed in the focal region of an aplanatic system. This can be quantified as

$$
\mathbf{E}=\left(e_{x} e_{y}, e_{z}\right)
$$

with

$$
\begin{aligned}
& \mathrm{e}_{x}(\mathbf{r})=-i\left(I_{o}+I_{2} \cos 2 \Phi\right) \\
& \mathrm{e}_{y}(\mathbf{r})=-i I_{2} \sin 2 \Phi \\
& \mathrm{e}_{z}(\mathbf{r})=-2 I_{1} \cos \Phi
\end{aligned}
$$

where $\Phi$ defines the azimuth angle between the initial direction of oscillation of the incident field and the direction of observation; $\mathbf{r}$ is the coordinate originating in the geometrical focus; and $I_{o}, I_{1}$, and $I_{2}$ are integrals over the objective lens aperture, depending on the aperture angle and the wavelength. From this the time-averaged illumination-intensity distribution in the focal region (i.e. the illumination pointspread-function, denoted as $\left.\mathrm{h}_{\mathrm{ill}}\right)$ ) for linearly polarized illumination was calculated from $I=|\mathbf{E}|^{2}=\left|I_{0}\right|^{2}+4\left|I_{1}\right|^{2} \cos ^{2} \Phi+\left|I_{2}\right|^{2}+2 \cos 2 \Phi \operatorname{Re}\left(I_{0} I_{2} *\right)$ for a large numerical aperture $(N A=1.4)$ and a given wavelength (e.g. $\lambda=633 \mathrm{~nm})$.

This illuminating intensity distribution is proportional to the probability to excite a fluorophore at a given site $(x, y, z)$ in the focal region.

The next step was to determine the detection point-spread function which is proportional to the probability to detect at a given site in the image plane a fluorescence photon emitted at a given site in the focal plane. For this, Hell \& Stelzer assumed that the fluorescent light is randomly polarized and (due to the Stokes shift) has a somewhat larger wavelength (in this case $725 \mathrm{~nm}$ ), but else the calculations were analogous to those to determine the illumination PSF (denoted as $h_{\text {det }}$ ).

The normalized confocal point-spread function (denoted as $\mathrm{H}$ ) was then given by the probability to detect at a given site a photon emitted there. Hence, the overall probability to excite and to detect a photon was given by the product of the probabilities for excitation and detection, i.e.

$$
\mathrm{H}=\mathrm{h}_{\mathrm{ill}} * \mathrm{~h}_{\text {det. }} .
$$

This approach allowed to calculate the 3D PSF of various types of 4Pi laser scanning confocal microscopes (and hence the achievable optical resolution in $(x, y, z)$ ) under special conditions. Hell and Stelzer noted that apart from operating as a confocal fluorescence microscope, the $4 \mathrm{Pi}$ confocal fluorescence microscope comprises three different types of imaging that feature a higher resolution:

Type A: The two illumination wave fronts interfere in the sample (4Pi illumination); Type B: The two detection wave fronts interfere in the detector (4Pi detection);

Type C: Both the illuminating and the detection wave fronts interfere (4Pi illumination and $4 \mathrm{Pi}$ detection).

All three types of 4Pi microscopy have been experimentally realized [Hell et al. 1994a,b; Hänninen et al. 1995; Gugel et al. 2004]. With few exceptions, however, so far only type A (4Pi illumination) has been used on a broader scale, due to the extremely small optical path differences required to realize constructive interference of fluorescent light. 
The 4Pi-microscopy concept [Hell and Stelzer 1992a,b] described above assumed single photon excitation of fluorescence. The numerical calculations as well as experimental results [Hell \& Stelzer 1992a; Hell et al. 1994a] showed, however, that in this way the axial FWHM of the central peak of the 4Pi-PSF was indeed narrowed to about one fourth of the CLSM value; but the side lobes remained in the order of more than $40 \%$ of the central peak maximum, posing a substantial obstacle to correction by deconvolution. This problem was shown to be overcome in $4 \mathrm{Pi}-\mathrm{C}$ microscopy [constructive illumination and constructive detection]. However, the technical realization of 4Pi-C microscopy is quite complex. In a second paper of the same year [Hell and Stelzer 1992b], a solution was described by the design of a 4Pi-confocal fluorescence microscope using two-photon excitation. In this arrangement, two photons are needed simultaneously for excitation, and in this case the illumination point-spread function $h_{\text {ill }}$ is given by $h_{\text {ill }} 4$ pi two phot $=\left[\text { hill }_{4 \mathrm{Pi}}\right]^{2}$. Hell \& Stelzer showed that in this case, the height of the side lobe peaks of the axial FWHM can be substantially reduced even in a $4 \mathrm{Pi}-\mathrm{A}$ arrangement (constructive illumination only).

In the following decade, confocal laser scanning 4Pi fluorescence microscopy applying either continuous wave visible laser light for single-photon excitation, or femtosecond pulsed infrared laser wavelengths for two- photon excitation [Hell et al. 1994a,b; Lindek et al. 1994; Hänninen et al. 1995] has become an established "focused nanoscopy" method and the first example of "Point-Spread-Function Engineering" which has been made commercially available [Egner et al. 2002; Hell 2003; Bewersdorf et al. 2006; Baddeley et al. 2006; Lang et al. 2010]. An axial optical resolution down to the $100 \mathrm{~nm}$ regime was experimentally realized, i.e. about five times better than in conventional confocal laser scanning fluorescence microscopy (CLSM). Although due to the sterical angle $<4 \pi$ realized by the two opposing objective lenses the optical resolution in the lateral direction was improved only slightly, the observation volume $V_{\text {obs }}$ used as a measure for 3D resolution [Hell and Stelzer 1992b; Lindek 1994, 1996] was substantially reduced compared with the conventional confocal case [Cremer 2012].

It has been argued that increasing the sterical aperture angle to values beyond $2 \pi$ as conceived in $4 \pi / 4 \mathrm{Pi}$ microscopy did not really "break" the Abbe limit (or the Rayleigh limit, repectively), because these concepts followed the basic idea of Abbe/Rayleigh that by increasing the aperture angle, the resolution can be improved [Stelzer 2002]. However, from the original papers of Abbe and Rayleigh it is also evident that they firmly believed that an increase of the aperture angle of the incident light beyond $\alpha / 2=90^{\circ}$ (and hence $\sin [\alpha / 2]=1$ ) was physically not possible. For about a hundred years, this view has become general textbook knowledge. It was only with the advent of coherent light sources in the 1960 s that concepts of $4 \pi / 4 \mathrm{Pi}$ microscopy became feasible.

\section{Stimulated emission depletion (STED) microscopy}

In experimental 4Pi-microscopy using two opposed objective lenses, so far a 3D optical resolution of around $200 \times 200 \times 100 \mathrm{~nm}^{3}$ has been realized. This means that objects with spatial features below about $100 \mathrm{~nm}$ would still remain unresolved. The first successful concept to achieve a lateral optical resolution far below $100 \mathrm{~nm}$ in a far-field arrangement using a single objective lens was STimulated Emission Depletion (STED) Microscopy conceived by Stefan Hell [Hell and Wichmann 1994; for a simultaneous invention see Baer 1994] and realized by Hell and his laboratory in the following decade.

This focused nanoscopy method is again based on scanning the object with a focused laser beam using a single high $N A$ lens [Schrader et al. 1995]. 


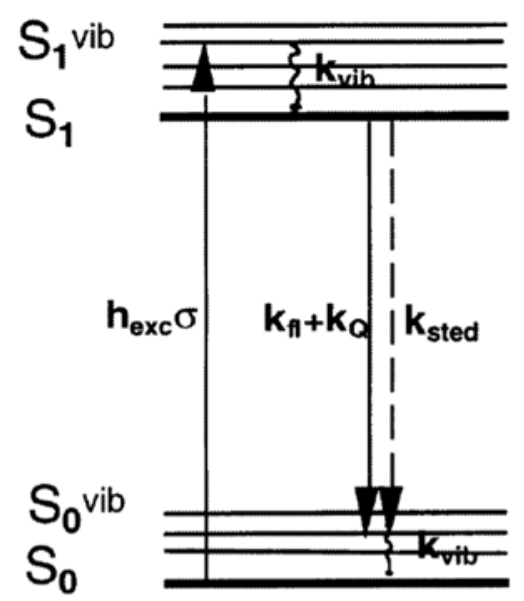

Fig. 3. Principle of STED microscopy. Shown are the energy levels of a typical organic fluorophore. $S_{0}$ and $S_{1}$ are the ground and first excited singlet states, respectively. $S_{0}^{\text {vib }}$, and $S_{1}^{\text {vib }}$ are higher vibronic levels of these states. The excitation of the dye takes place from the state $S_{0}$ to the state $S_{1}^{\text {vib }}$, and fluorescence by the radiative transition $S_{1}$ to $S_{0}^{\text {vib }}$. The transition $S_{1}$ to $S_{0}^{v i b}$ can also be induced by stimulated emission. For sufficiently high intensities of a stimulating beam a significant depopulation (Stimulated Emission Depletion/STED) of the $\mathrm{S}_{1}$ level can occur. Fluorescence has a broad spectrum extending over several tens of nanometers in wavelength but the stimulated photons have the same wavelength, polarization and direction of propagation as their stimulating counterparts. Thus, photons with the same characteristics as the STED beam can be eliminated from the fluorescence signal detected. From [Schrader et al. 1995], with kind permission from IOP Publishing Ltd.

However, in spite of increasing the sterical aperture angle beyond $2 \pi$, in this case the basic idea was to realize enhanced resolution by an optical device which allowed to register the fluorescence emission from a much smaller object region than given by the diameter $\left(\varnothing_{\mathrm{ill}}\right)$ of the illuminating focused laser beam. This was achieved by an appropriately shaped second laser beam of wavelength $\lambda_{\text {STED }}$ (the STED beam) producing an illumination pattern around the excitation beam $\left(\lambda_{\text {exc }}\right)$ by which excited molecules $\left(S_{1}\right.$ state $)$ in the vicinity $\left(d \ll \emptyset_{\text {ill }}\right)$ of the center of the focus of the excitation beam were induced to stimulated emission of radiation from the lowest $\mathrm{S}_{1}$ state to the $\mathrm{S}_{0}^{\text {vib }}$ ground state (Figure 3). By appropriate excitation/detection arrangements, this emission was separated from the spontaneous fluorescence emission (at wavelengths $\lambda_{\text {fluor }}$ ) of the molecules in or very close to the focal center of the excitation beam; these were not subjected to the - typically doughnut shaped - STED beam ideally producing a zero intensity point just in the center of the focus of the exciting beam (having a diameter $\varnothing_{\text {ill }}$ ). As a consequence, the stimulated emission of these molecules was not registered by the detector system set to detect the spontaneous fluorescence $\left(\lambda_{\text {fluor }}\right)$ only. In a next step, either the object or the two coupled beams are moved to a neighbouring site, and the procedure is repeated. In this case, the molecules previously positioned in the center of the exciting beam $\left(\lambda_{\text {exc }}\right)$ are now stimulated to emission by the STED beam $\left(\lambda_{\text {STED }}\right)$ and thus do not contribute to the spontaneous fluorescence signal $\lambda_{\text {fluor }}$; the molecules previously stimulated to emission by the STED beam are now in the center of the exciting beam, and their fluorescence signal $\lambda_{\text {fluor }}$ is registered. The molecules excited to fluorescence at $\lambda_{\text {fluor }}$ can be denoted as being in a 'bright' or 'ON' state with respect to the detector system while the molecules induced to stimulated emission can be denoted as being in a 'dark' or 'OFF' state. 
STED microscopy is a first example for a general spatially scanning nanoscopy concept (see below) involving focused or structured illumination with zero intensity positions in which the fluorescence emission $\left(\lambda_{\text {fluor }}\right)$ is registered first only from an area $\mathrm{A}$ with $\varnothing_{\text {fluor }} \ll \varnothing_{\text {ill }}$ and then the fluorescence emission is registered only from an area B with $\varnothing_{\text {fluor }} \ll \varnothing_{\text {ill }}$ with a distance $d_{\mathrm{AB}} \ll \varnothing_{\text {ill }}$; and the object positions $\mathrm{A}$, B are obtained by the mechanics of the scanning procedure.

By appropriate saturation intensities, the fluorophores in a doughnut shaped annulus around the center of the exciting laser focus are induced to emit photons by stimulated emission of radiation at a red shifted wavelength compared to the fluorescence emission maximum. The remaining fluorescence, which can be spectrally separated from the high intensity STED beam, can thus only originate from the center where (in the ideal case) the doughnut shaped STED beam has a zero intensity point. As a consequence, fluorescence photons of a given energy are detected from a region with area much smaller than $\emptyset_{\text {ill }}$. Since due to the scanning mechanism, the position of this smaller fluorescent region can be known with an accuracy of few nm (and even smaller), the fluorescence signal obtained can now be assigned to this smaller region; hence the optical resolution may be enhanced further. STED microscopy as reviewed in a recent review article was the first implementation of utilizing nonlinear response in enhanced resolution fluorescence microscopy [Cremer 2012].

Later implementations of the STED microscopy concept yield enhanced resolution imaging in the object plane $(x, y)$ and presently has found numerous applications [Willig et al. 2006, 2007; Nagerl et al. 2008; Westphal et al. 2008]. The best (lateral) optical resolution values obtained by STED microscopy are presently around 15 to $20 \mathrm{~nm}$ in biomedical applications [Donnert et al. 2006] and $\sim 6 \mathrm{~nm}$ in solid state physics [Rittweger et al. 2009]. In a combination with the 4Pi-method, a 3D optical resolution of few tens of nanometers was achieved [Schmidt et al. 2008].

From a historical point of view it may be noted, that in the same year (1994) when the basic conceptual idea underlying STED had been put forward by Hell and Wichmann [Hell and Wichmann 1994] (received by the Editor March 7, 1994), a similar concept was submitted as a patent application (however, without the detailed calculations reported by [Hell and Wichmann 1994]) to the United States Patent office by Stephen C. Baer [Baer 1999]. Baer's patent on "Method and apparatus for improving resolution in scanned optical system" was filed in the United States on July 15, 1994, and the patent date is February 2, 1999. According to Baer's patent, this patent is for a zero-point STED microscope and may be consulted (as well as his other patents on superresolution optical microscopy) on the web site of the United States Patent and Trademark Office (www.uspto.gov) for Stephen C. Baer.

As a general problem attribution questions are sometimes difficult to clarify when the inventor published in a medium (e.g. a highly specialized journal) that is not readily available or in a language that is unknown to the author of the review article. Often the patent literature is ignored as a source of attribution of credit. Until the advent of suitable electronic screening techniques, this neglect was mostly justified because of the difficulty for a scientist to even know the existence of the publication in question. For example, the now famous US patent application of Marvin Minsky (filed November 7, 1957) on the construction of a confocal microscope (based on transmitted/reflected light with a non-coherent light source) for decades was buried in the US patent office under the heading "Microscope apparatus." Hence it was not cited until its "rediscovery" in the 1980s. Only with the development of the laser as a light source and the desk top computer as a system controller did the confocal microscope attract the attention of cell biologists [Masters 1996]. Another problem is the fact that independent inventors who lack an academic affiliation are sometimes excluded from important review articles. A remedy for this may be the publication of 


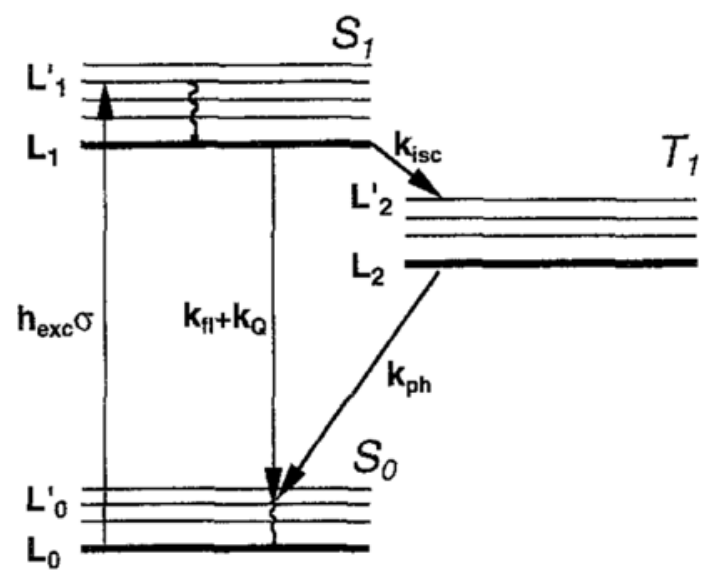

Fig. 4. GSD microscopy using focused illumination. Shown are the energy states of a typical fluorophore. $S_{o}$ is the ground state and $S_{1}$ the first singlet state; $T_{1}$ is the first triplet state; $L_{o}$ is a low vibrational level of $S_{o}, L_{1}$ the vibrationally relaxed state in $S_{1}, L_{2}$ a vibrationally relaxed level of $T_{1}$ and $\mathrm{L}^{\prime}$ a vibrationally higher level. For appropriately high laser intensities (in the order of $10 \mathrm{MW} / \mathrm{cm}^{2}$ ), most molecules are trapped in the long-lived triplet state, $T_{1}$, and the ground state is depleted. $\mathrm{S}_{0}$ remains depleted as long as the focused excitation beam is switched on. From [Hell and Kroug 1995], with kind permission from Springer Verlag.

books on a given subject that contain selected papers and patents that are reprinted in full [Masters 1996; 2001; 2003].

\section{Ground-state-depletion (GSD) microscopy [Hell and Kroug 1995]}

In 1995, a very elegant alternative was proposed by [Hell and Kroug 1995] to use the same general principles as in STED microscopy but instead of depleting the excited state $\left(S_{1}\right)$, the ground state $\left(S_{0}\right)$ of the fluorescence molecules in the outer region of the excitation focus $\left(\lambda_{\text {exc }}\right)$ is depleted (Figure 4$)$. The general basis for such a ground-state depletion is the control of excitation made possible by focused short laser pulses.

For example, phase-shifted, low-energy picosecond or subpicosecond pulse trains make it possible to modulate excitation; or, suppression of excitation is possible by phase-shifting of two lasers [Warren et al. 1993].

Thanks to these novel approaches to control molecular quantum dynamics, in the GSD concept the illumination conditions (i.e. the intensity of the depletion beam) are set up in such a way that the density $\left(n_{0}\right)$ of molecules in an $\mathrm{S}_{0}$ state is greatly diminished in the outer region of the focus; hence, these molecules cannot be transferred to an $\mathrm{S}_{1}$ state, and they cannot emit photons by a $\mathrm{S}_{1}$ to $\mathrm{S}_{0}$ transition. For imaging by this method, it is essential that by moving the scanning laser focus to another site and by reducing the intensity of the depletion beam the molecules are quickly relaxed to the $\mathrm{S}_{0}$ state. That means that the depletion process has to be reversible on a sufficiently fast time scale $(\mu \mathrm{s})$.

To realize GSD based focused nanoscopy [Hell and Kroug 1995] proposed the use of two focused laser beams being symmetrically offset by a small value with respect to the geometrical focus. In the numerical example given, the molecules at the regions surrounding the point of interest are first exposed to a higher intensity beam depleting the ground state (in the intensity range of $1 \mathrm{MW} / \mathrm{cm}^{2}$ ). After about $1 \mu$ s the depletion beam is switched off, and after $\sim 5$ ns nearly all the molecules from the first singlet 
state are relaxed; but for a time interval of about $0.2 \mu \mathrm{s}$, the molecules being caught in the triplet state have still not returned to the ground state and thus cannot be excited by the second probing beam of lower intensity As a result, the molecules in the inner region of the focal area subjected only to low illumination intensities can rapidly cycle between $S_{0}$ and $S_{1}$, emitting a photon at every $S_{1}$ to $S_{0}$ return (on the average every few ns, in contrast to the $1000 \mathrm{~ns}$ range for the $T_{1}$ to $S_{0}$ transitions). Consequently, the effective area from which photons are registered by the detector was calculated to be greatly diminished compared to $\varnothing_{\text {ill }}$.

Assuming an excitation/depletion wavelength of $400 \mathrm{~nm}$ and a numerical aperture of $N A=1.4$, [Hell and Kroug 1995] estimated a lateral optical resolution (FWHM of the effective PSF) of about $15 \mathrm{~nm}$.

The concept of the single point scanning GSD microscope outlined above was experimentally realized in 2007 [Bretschneider et al. 2007]. In these proof-of-principle experiments using a confocal stage scanning microscope, relatively modest depletion intensities $\left(\lambda_{\text {exc }}=532 \mathrm{~nm} ; I_{\text {depl }} \sim 10^{2} \mathrm{~kW} / \mathrm{cm}^{2}\right)$ in a focal distribution featuring a local intensity zero were sufficient to confine the fluorescence emission of rhodaminelike fluorophore (Atto532) molecules to a very small region around the local intensity zero; a lateral optical resolution (FWHM of the effective FWHM) down to ca. $50 \mathrm{~nm}$ was achieved. As a first application example for this type of scanning GSD microscopy, Atto532-stained microtubules and membrane bound protein clusters were imaged, featuring a clear improvement compared to confocal images of the same structure [Bretschneider et al. 2007].

\section{Reversible saturable optical fluorescence transitions (RESOLFT) microscopy}

During the last decade (2000-2010), the concepts outlined above for STED-microscopy and GSD microscopy have been generalized by Stefan Hell to be valid for an entire class of scanning nanoscopy approaches, called RESOLFT microscopy (REversible Saturable OpticaL Fluorescence depletion Transitions) [Hell 2007, 2009]. The RESOLFT principle [Hell 2007] stated that enhanced resolution by any kind of scanning device can be obtained if illumination conditions can be realized with focal spots (or other intensity distributions) featuring central intensity zeros which allow to inhibit transiently the reversible fluorescence emission of molecules outside this zero region, e.g. by stimulated emission, or by ground state depletion.

To achieve enhanced resolution in the RESOLFT scheme, the fluorophore molecules used generally have to allow light induced reversible transitions between two states A and B at any point in space. In the simplest case, A and B are conceived as energy states of this entity, such as a ground and an excited state. Other A to B transitions considered were molecular conformational states, photochromic and isomerization states, binding and protonation states, etc. The transition $\mathrm{A} \rightarrow \mathrm{B}$ is assumed to be photoinducible while no additional restrictions are needed about $\mathrm{B} \rightarrow \mathrm{A}$. In the most general case, the transition $\mathrm{B} \rightarrow \mathrm{A}$ was assumed to provide a spontaneous component together with a component which is triggered externally through light, heat, by a chemical reaction. To be practical, the reversible dark-bright transition cycle $\mathrm{A} \rightarrow \mathrm{B}, \mathrm{B} \rightarrow \mathrm{A}$ are conceived to be fast enough to allow efficient 'point-by-point' or 'line-by-line' scanning, i.e. the transitions have to be in the order of $\mu$ s to ms.

An essential requisite of the RESOLFT concept is that scanning is used, i.e. either the object is moved in a specific way, or the position of the illumination pattern relative to the object is changed to move the focal spots with central zeros to different sites of the object.

As a general formula for the resolution [Schwentker et al. 2007] the relation

$$
\Delta x \approx \lambda /(2 n \sin \alpha \sqrt{1+\xi})
$$


is stated, where $\Delta x$ is the smallest distance detectable (optical resolution) with a point scanning system with central zeros, $\lambda$ is the excitation wavelength, nsin $\alpha$ is the numerical aperture $(N A)$ and $\alpha$ is the half aperture angle of the objective lens used. The saturation factor, $\xi=\mathrm{P} /$ Psat is the applied power $\mathrm{P}$ driving the reversible, light-driven transition between a fluorescent and a nonfluorescent state of the dye label with a focal intensity distribution with a local zero divided by the saturation power $\mathrm{P}_{\mathrm{SAT}}$ that classifies the magnitude of the power necessary to attain $50 \%$ of the transition.

In the description given by [Hell 2007], it was noted that for $\xi=0$ (high saturation intensity compared to the applied intensity), i.e. for linear excitation conditions, the Abbe equation for two-point resolution is obtained. The higher the saturation factor becomes, the more the optical resolution is improved.

A further generalization of the RESOLFT concept of far-field resolution enhancement was proposed. This approach can be applied not only to single point scanning systems (one focal spot featuring a central zero as in the basic concepts of STED [Hell and Wichmann 1994] and GSD microscopy, [Hell and Kroug 1995] but to other scanning schemes based on illumination conditions creating intensity distributions that feature zero intensity sites. For example, scanning schemes with multiple "spots" or zero intensity lines as realized in Saturated Patterned Excitation Microscopy [Heintzmann et al. 2002; Schwentker et al. 2007] can also be used to enhance resolution.

\subsection{Structured illumination excitation (SIE) microscopy}

In contrast to resolution enhancement by focused nanoscopy approaches, the use of appropriately modified illumination patterns for fluorescence excitation has the major advantage of avoiding point-by-point scanning and detection of the object. This allows a substantial increase of the imaging speed. While the optical resolution (in the sense of cutoff frequency of the OTF) using linear excitation modes can only been doubled [for first theoretical concepts see Lukosz 1966, 1967], under special conditions the size of optically isolated objects can be determined down to a small fraction of the exciting wavelength applied (see below, SMI microscopy).

\section{Standing-wave fluorescence microscopy (SWFM)}

In the beginning of the 1990s, the first description of a standing wave microscope was reported [Bailey et al. 1993] where two coherent collimated laser beams interfered in the object space in such a way that a standing-wave excitation of fluorescence was produced. A technique to generate the desired illumination pattern was to bring two counter-propagating laser beams to interference, establishing a standing-wave field. The standing waves formed by this interference created an excitation field with closely spaced nodes and antinodes, which provides imaging of the specimen with a very high discrimination along the optical axis (down to $\sim 50 \mathrm{~nm}$ ). The technique was used to obtain images of actin fibres and filaments in fixed cells, actin single filaments in vitro and myosin II in a living cell [Bailey et al.1993].

In an accompanying paper [Lanni et al. 1993], the authors compared the axial resolution attainable by SWFM to conventional methods of fluorescence optical sectioning microscopy (FOSM). They concluded that "a field shift as small as one-quarter of the node spacing was found to cause readily-interpretable changes in the image, suggesting that the axial resolution can be better than $\lambda / 8 n$, or $0.04-0.05 \mu \mathrm{m}$, an order-of-magnitude improvement over focused scanning optical microscopy FOSM." 


\section{Spatially modulated illumination microscopy (SMI)}

Spatially Modulated Illumination (SMI) microscopy introduced in the mid-1990s [Hausmann et al. 1997] is a method of wide-field fluorescence microscopy using axially structured illumination for fluorescence excitation. The optical method to create structured illumination along the optical axis was practically the same as in the SWFM approach of Bailey et al. (1993). In contrast to Bailey et al., quantitative high resolution information about sizes and relative positions of fluorescently marked target regions was obtained by moving the object in precise small steps (e.g. $20 \mathrm{~nm}$ ) along the optical axis to register the fluorescence emission at each step by a highly sensitive wide field camera, and to extract information from the quantitative combination of the multiple image frames thus obtained.

In contrast to focused laser light techniques [Hell et al. 1994, Egner et al. 2002; Hell $2003 ; 2007$ ] or to other types of structured illumination with excitation intensity modulation along the object plane [Gustafsson 1999, 2000, 2005; Gustafsson et al. 2008; Frohn et al. 2000; Heintzmann and Cremer 1999, Heintzmann et al. 2002], the SMI method as originally described [Hausmann et al. 1997; Schneider et al. 2000] was not suited for the generation of optical resolution enhanced images (in the meaning of an improved two-point resolution or of a narrower FWHM of a PSF with sufficiently low side lobes to allow deconvolution, like in [Hänninen et al. 1995]) of photostable fluorescence emitters. This was due to a range of missing spatial frequencies in the standing wave field generated by the two counterpropagating collimated light laser beams.

However, in combination with high-precision axial positioning this technique of farfield light microscopy allowed the nondestructive high precision localization analysis of complex spatial arrangements [Albrecht et al. 2001, 2002] inside relatively thick transparent specimens such as the cell nucleus and enabled size measurements at molecular dimensions of a few tens of nanometers [Failla et al. 2002b, 2003; Martin et al. 2004; Baddeley et al. 2010] and 3D position measurements down to the $1 \mathrm{~nm}$ range [Albrecht et al. 2001, 2002; Failla et al. 2001; Baddeley et al. 2007]. It may be noted that the SMI precision localization measurements were explicitly seen by the authors as a step towards resolution enhancement by localization microscopy [e.g. Albrecht et al. 2001, 2002] (see below).

SMI microscopy has been developed to an established method for the size analysis of small protein clusters and chromatin domains [Martin et al. 2004; Mathée et al. 2006; Hildenbrand et al. 2005; Birk et al. 2007; Baddeley et al. 2009]. In addition, it has been shown to allow nano-scale measurements in the living cell nucleus [Reymann et al. 2008].

\section{Patterned/structured illumination microscopy (PEM/SIM)}

The first experiments to overcome the conventional optical resolution limit in the lateral direction (object plane) by a single objective lens approach and wide-field based fluorescence detection (e.g. using CCD cameras, in contrast to point detectors like photomultipliers or avalanche diodes) were performed by the end of the 1990s [Heintzmann \& Cremer 1999; Gustafsson et al. 1999; 2000]; these methods have presently found a variety of biomedical applications [Schermelleh et al. 2008; Brunner et al. 2011; Best et al. 2011; Markaki et al. 2010].

The basic principle of these approaches was to create a spatially modulated illumination pattern not only in the axial direction $(z)$ as in Standing Wave Microscopy [Bailey et al. 1993] or in SMI microscopy [Hausmann et al. 1997; Schneider et al. 2000], but also in the object plane $(x, y)$. This can be done e.g. by inserting a diffraction grating in the illumination beam at the conjugate object plane and projecting it 
through the objective lens into the object. Instead of a diffraction grating one may create the desired illumination pattern also by the interference of two or more laser beams [Frohn et al. 2000; Gustafsson 2005; Schermelleh et al. 2008; Best et al. 2011]. The object and the illumination pattern are then moved relative to each other in precise steps. At each step, a wide-field detection image is taken by a CCD camera. The images obtained are used to calculate an image with enhanced resolution using an algorithm based on the structure of the Fourier space. Principally, the effective optical resolution can be improved up to a factor of two compared with conventional wide-field microscopy.

\section{Saturated patterned excitation microscopy (SPEM)}

In the wide-field based enhanced resolution schemes so far presented, a linear relation between excitation intensity and fluorescence emission is assumed. This is the typically desired condition in conventional fluorescence microscopy, where non-linear effects like saturation and photobleaching usually are avoided. Nonlinear effects, however, can be used to increase the optical resolution in Point Spread Function engineered focused scanning methods as in STED- or GSD microscopy, and can give highly interesting possibilities in structured illumination excitation approaches. A comprehensive review of nonlinear optics in microscopy is useful to gain a deep insight to this topic [Masters and So 2008].

In 2002, it was proposed to use nonlinear patterned excitation schemes to achieve a substantial further improvement in optical resolution by deliberate saturation of the fluorophore excited state [Saturated Patterned Excitation Microscopy/SPEM; Heintzmann et al. 2002]. The nonlinearity induced in this way leads to the generation of higher spatial harmonics in the pattern of emission, i.e. components in Fourier space beyond the frequency limit defined by the Abbe condition are created. The post acquisition manipulation of the acquired data is computationally more complex than in STED or GSD, but the experimental optical requirements are considerably more simple.

Computer simulations [Heintzmann 2003] showed that by appropriate extension of the algorithms developed for the case of linear structured illumination excitation a further improvement of optical resolution should be possible in SPEM. It is interesting to note that the sharp "emittability" zeros created by non-linear excitation patterns might be used as "virtual pinholes." This opened an avenue to apply the RESOLFT concept (see above) to nonlinear pattern based scanning devices; instead of realizing illumination conditions with focal spots featuring central zeros to inhibit transiently the reversible fluorescence emission of molecules outside this zero region, e.g. by stimulated emission, or by ground state depletion, in SPEM based RESOLFT scanning microscopy lines with central emission zeros are created [Schwentker et al. 2007]. In this way, in principle an 'unlimited' optical resolution should be possible not only in focused nanoscopy techniques with point-by-point fluorescence registration (e.g. by a photomultiplier, or an avalanche diode) but also in nonlinear structured illumination excitation (SIE) scanning approaches using wide-field registration (e.g. a sensitive CCD camera).

\subsection{Micro-axial tomography: a wide-field approach to enhance three-dimensional resolution}

In spite of the great progress obtained in optical resolution enhancement using focused nanoscopy and patterned excitation approaches, it remained highly desirable to consider in addition widefield solutions with improved 3D resolution using homogeneous 
illumination. The first approach towards this goal has been to apply the tomography method well known from electron microscopy to a fluorescence based wide field microscope. In this way, it seemed to be possible to numerically combine images taken from different angles in such a way that it becomes possible to obtain a 3D image with an isotropic optical resolution corresponding to the lateral resolution, i.e. $\sim 200 \mathrm{~nm}$. This would result in approximately three times enhancement of the 3D observation volume [Heintzmann and Cremer 2002].

To achieve this, micro-axial tomography was suggested which allows object tilting on the microscopic stage. Micro-axial tomography makes use of special glass capillaries [Bradl et al. 1992, 1994] or glass fibers [Bradl et al. 1996] as specimen carriers. This yielded an automated multi-view 3D image acquisition and precise 3D image alignment of different perspectives of the same objects. So far, micro-axial tomography has been applied to 3D studies of fluorescence labeled cellular structures [Heintzmann and Cremer 2002] using a setup with an external stepping motor and a flexible shaft, and also to perform highly precise measurements of focal depth dependent chromatic shifts [Edelmann et al. 1999]. Recently, a miniaturized device has been presented which can be implemented in a motor driven microscope stage [Staier et al. 2011].

\subsection{Superresolution optical fluctuation imaging (SOFI)}

The development of switchable fluorescent probes (see section 5.5, below) also triggered the emergence of an enhanced resolution method based on the analysis of temporal fluorescence fluctuations of emitters (e.g., fluorescence intermittency), called superresolution optical fluctuation imaging (SOFI). Enhanced resolution by SOFI [Dertinger et al. 2009, 2010a,b; has been demonstrated by using a conventional widefield microscope with homogeneous illumination equipped with a CCD camera, but the principle can be applied to any kind of fluorescence imaging method (spinning disk, scanning confocal, total internal reflection, etc.). SOFI relies on the registration of independent stochastic fluctuations of the fluorescence emitters.

To achieve enhanced resolution (up to two times improvement compared to the Abbe-limit), three main conditions must be met:

1. The fluorescent label has to exhibit at least two different emission states. For example, these states can be a fluorescent and a nonfluorescent one, but in principle any two or more states that are optically distinguishable will do.

2. Different emitters have to switch between states repeatedly and independently from each other in a stochastic way.

3. For this approach, the image should be acquired with pixels smaller than the diffraction limit.

\subsection{Localization microscopy approaches}

As outlined above, the absolute limits of light-optical resolution were theoretically well established. To substantially overcome the conventional limits, focused nanoscopy approaches and (up to a factor of two) structured illumination techniques appeared to be the only way out. Using homogeneous illumination, however, an enhanced resolution appeared to be strictly impossible, due to very fundamental principles of electromagnetic wave theory. A careful examination of these principles reveals however, that they consider always the case that the point sources to be resolved have the same time independent emission spectrum; i.e. the photons emitted by them cannot be 'sorted' according to their source. It is evident that as soon as such a 'sorting' is possible, new possibilities of enhanced resolution can be created. Nonetheless, effective methods of enhanced resolution in epifluorescence light microscopy based on this principle 
have been developed only in the last few decades. This late development of localization microscopy may be due to many reasons, such as the deeply rooted general conviction that an enhanced resolution even in the case of homogeneous illumination is physically impossible; the lack of suitable light sources, sufficiently sensitive detectors, mechanically ultrastable microscope stages, as well as appropriate and fast evaluation algorithms; the lack of appropriately labeled objects; more generally, the lack of knowledge about the photophysics of molecules; as well as the many technical obstacles to the experimental realization of the apparently evident and simple basic idea of optical isolation and localization of fluorescent point sources.

Localization microscopy approaches presently achieve a two-point resolution of adjacent single molecules down to the $1 \mathrm{~nm}$ range, corresponding to ca. one five hundredth of the exciting wavelength [Pertsinidis et al. 2010], and a structural resolution (multiple molecules localized within a single Airy disc area) in the range of few tens of $\mathrm{nm}$.

\section{Principles of localization microscopy}

Generally, localization microscopy (for reviews see [Zhuang 2009; Cremer et al. 2011; Cremer 2012]) is based on the optical isolation of point emitters, their subsequent high precision localization, and assignment of these coordinates to a joint position map; the optical isolation is achieved by using a suitable set of optically detectable physical characteristics, such as differences in absorption/emission spectra [Betzig 1995; Ha et al. 1996; Cremer et al. 1996, 1999; Bornfleth et al. 1998; van Ojen 1998; Esa et al. 2000, 2001], fluorescence life times [Cremer et al. 1996, 1999, Heilemann 2002], and time dependent differences in luminescence [Cremer et al. 2002]; or various methods of photoswitching of single molecules between two optically distinguishable states, e.g. a 'dark' and a 'bright' state [Hell and Kroug 1995; Lidke et al. 2005; Betzig et al. 2006; Hess et al. 2006; Rust et al. 2006; Andresen et al. 2008]. Recently, the application range of such localization microscopy methods has been greatly extended by the use of standard single fluorophores, e.g. [Bock et al. 2007; Reymann et al. 2008; Lemmer et al. 2008; Heilemann et al. 2008; Fölling et al. 2008; Baddeley et al. 2009a,b, 2011; Zhuang et al. 2009; Markaki et al. 2010; Kaufmann et al. 2012a,b]. From today's perspective, the basic principles of localization microscopy as outlined above appear to be almost self evident, and their realization seems obvious. This, however, has not always been the case:

1) A first fundamental problem for the development of localization microscopy was the century long tradition of Fourier analysis to conceive an object to be optically represented by a large number of harmonic functions of different spatial frequencies; the main task of microscopy was then to transfer spatial frequencies as high as possible; the resolution achievable was represented by the Optical Transfer Function/the Contrast Modulation Function and its spatial cut-off-frequency, i.e. by the highest number of lines/ $\mu \mathrm{m}$ still discernible in the image. This, for example, was the basis for the analysis of resolution by Ernst Abbe (1873). The impact of this tradition is well documented by a large number of textbooks on optics dealing with the problem of resolution.

2) A second fundamental obstacle for the development of localization microscopy was the firm belief that the Abbe and the Rayleigh theory of resolution were essentially equivalent. This meant that the fundamental difference resulting from the interaction of light with molecules in the excitation of fluorescence/luminescence was not recognized. Even in modern textbooks of optics, such interactions often are mentioned only very briefly; the photophysics of molecules was regarded to be a branch of physical chemistry, not of optics. 
3) A third fundamental obstacle to the development of localization microscopy was that the large variety of interaction modes of light with matter and the resulting large spectrum (in the general sense as a distribution of physical characteristics) of possible responses was overlooked.

4) A fourth fundamental obstacle has been the lack of specific molecular labeling techniques. For example, a method permitting the imaging of all molecules in a cell with a nanometer optical resolution would not be very interesting for biologists: what they want to know is the spatial distribution of specific molecules, e.g. of certain types of proteins, or of specific DNA sequences. This is possible only if such molecules can specifically be labeled with a fluorescent marker. Such labeling methods became generally available only within the last decades (for a review see [Cremer and Cremer 2001]).

5) In addition, a variety of basic technical problems had to be overcome to make localization microscopy a meaningful alternative to other types of microscopy with enhanced resolution [Cremer et al. 1999]. For example, localization microscopy of single molecules using homogeneous illumination schemes required highly sensitive and fast detectors, such as CCD-cameras or CMOS arrays, to effectively register the relatively small number of photons (also as a function of time) emitted by a single molecule. It required the insight (nowadays evident but until a decade ago not well appreciated) that a localization of a molecule can be performed with an accuracy values orders of magnitude smaller than the pixel size of the detector. It required methods to discriminate the few photons emitted by a molecule from the photons emitted by the underlying background and it required a high mechanical stability of the optical system not realized in most conventional microscopes. In addition it required very precise calibration methods to overcome the chromatic and monochromatic aberrations inherent to all optical systems composed of glass lenses; last but not least, localization microscopy also required the development of sufficiently fast evaluation algorithms implemented on suitable desktop computers to allow the fast and precise determination of molecule positions from the analysis of typically many thousands of relatively "noisy" signals, major problems being the background and the low number of photons detected. For example, for the development of localization microscopy algorithms reported by [Bornfleth et al. 1998], a state-of-the-art 200 MHZ Silicon Graphics workstation with 96 MB RAM had to be used, with correspondingly slow performance. In contrast to this, presently used PCs and algorithms allow the assignment of several thousand molecule positions per second [Grüll et al. 2011].

For all these reasons, the development of localization microscopy has been much slower than one would expect from today's perspective. Furthermore, it is interesting to note that the development of localization microscopy has taken place in a relatively small number of experimentally working and often interconnected pioneer groups with a strong tradition in interdisciplinary research (Physics/Physical Chemistry/Biology) to overcome the various severe mental and technical obstacles mentioned.

Here, we attempt to briefly summarize these developments from a historical perspective. This appears to be justified not only from the historical point of view but also by the fact that in addition to published accounts, the exchange of ideas between most of the pioneer groups in this field has been well established for decades also in the context of countless congress presentations and numerous joint collaborations, including large funded projects. Therefore, attribution of credit to a very few individuals will be difficult; nonetheless, attribution to a few pioneer groups (without trying to rank their importance in the development of localization microcopy) appears to be possible. 
On the European side, a number of well interrelated pioneer groups (with PIs C. Cremer, S. Hell, M. Sauer, E. Stelzer) has been initially located at Heidelberg (EMBL, Heidelberg University), in close connection with the Max-Planck Institute of Biophysical Chemistry in Göttingen (since the 1990s). In this frame, joint collaborative funded projects on the German Research Agency and Federal Government level were performed. Some PIs of the Heidelberg area were also connected with the Sam Hess group at the University of Maine (joint appointments of S. Hess and C. Cremer at the Institute of Molecular Biophysics at University of Maine/The Jackson Laboratory since 2004).

The basic principle of 'localization microscopy' is applicable in a wide range of microscopy methods, even in the case of homogeneous wide-field illumination: the desired information about the position of point emitters (e.g. single fluorescent molecules) is derived directly from the diffraction pattern produced by such point emitters in the image plane which may be registered by any means, in particular including wide-field detectors without scanning. In contrast to the localization microscopy approach, in "focused nanoscopy" methods such as 4Pi, STED, GSD, SPEM, and generally in RESOLFT microscopy, a focal maximum or zero featuring intensity distributions are necessary to obtain an enhanced resolution. A highly nonhomogeneous intensity distribution of the excitation illumination in the object is required also in SIE microscopy modes based on patterned illumination.

Since the mid-1990s, a large variety of localization microscopy concepts has been developed and realized. They have been denoted as e.g.: BLINKING, FPALM, GSDM, PALM, RPM, SPDM, STORM, dSTORM, d ${ }^{4}$ STORM etc.

Very general ideas on related strategies for attaining enhanced resolution using spectroscopic data as constraints date back to the 1980s. For example, [Burns et al. 1985] considered the problem of precision localization of two point objects within the Rayleigh distance of each other, possessing different spectral emission characteristics. Assuming linear superposition of the spectral characteristics, they showed by computer simulations that by appropriate, rather complex algebraic methods and assuming a signal-to-noise-ratio (SNR) of $15 \mathrm{~dB}$, two point objects within a distance of $1 / 30$ to $1 / 50$ of the Rayleigh distance were still spatially resolvable. It was not clear, however, to what extent these arguments were practical to achieve enhanced resolution in far field fluorescence light microscopy, and especially if the number of point objects located in an area smaller than the diameter of the Airy disc was increased to significantly higher values.

This problem was treated by [Betzig 1995] who discussed possibilities to realize localization microscopy of multiple point sources localized within the Airy disc (i.e. with distances below the Rayleigh distance) in a near field optical scanning microscope at helium temperatures. Under these low temperature conditions, absorption cross sections would be sharp enough to achieve by appropriate laser based absorption line tuning the necessary optical isolation of adjacent molecules, i.e. the clear separation of their diffraction patterns. In contrast to the deconvolution procedure of [Burns et al. 1985], the evaluation of the independently registered ("optically isolated") diffraction patterns would allow to localize any number of point sources with mutual distances smaller than the conventional resolution. Experiments performed some years later by [van Ojen et al. 1998] showed that this concept indeed yielded enhanced resolution of multiple single molecules in low temperature far-field microscopy. Due to the technical restrictions of cooling the specimen down to a few Kelvin, only numerical apertures $N A<1.0$ were used.

[Hell and Kroug 1995] discussed optical isolation and localization of molecules in the vicinity of a focused laser beam in a far-field scanning microscope at approximately $300 \mathrm{~K}$ operating temperature by intensity induced reversible transitions between the ground state $\left(\mathrm{S}_{0}\right)$, the first excited state $\left(\mathrm{S}_{1}\right)$, and the triplet state $\mathrm{T}_{1}$; this concept 
was later integrated into the RESOLFT concept, indicating a possible mechanism for light induced reversible transitions between dark and bright states.

In December 1996, the Shimon Weiss group from University of California Berkeley [Ha et al. 1996] published a paper on "Dual-Molecule Spectroscopy: Molecular Rulers for the Study of Biological Macromolecules" in which they reviewed recent techniques and experiments based on the detection and spectroscopy of two near-by molecules including room-temperature, in connection with near field scanning optical microscopy (NSOM). In this paper, they discussed the possibility that if two closely adjacent molecules are distinguishable by one or more optical properties (emission color, radiative lifetime, dipole orientation), their location and the distance between them can be determined with high precision, down to the order of one hundredth of the FWHM of the PSF. They stated that "the main challenge in doing so is to separate the signal from the two molecules while maintaining perfect registry between them. Dual-color excitation with NSOM permits exactly that." The object "is scanned and two images are simultaneously acquired. Since both excitation colors share the same nearfield aperture, the two registered images are in perfect alignment. The centers of the two PSF's of the two labelling molecules is then determined and the distance between them is extracted."

At the same time (December 1996), [Cremer et al. 1996] proposed to implement a localization based mode of enhanced resolution microscopy applicable to any far-field microscopy method, including for the first time also fluorescence excitation of multiple point sources by homogeneous illumination, allowing the use of high $N A$ (larger 1.0) objective lenses and operating at room temperature. This concept of far field localization microscopy has been denoted as Spectral Precision Distance Microscopy (SPDM) by the authors [Cremer et al. 1999; Esa et al. 2000, 2001]. Compared with [Betzig 1995] and [Ha et al. 1996] the localization microscopy concept was extended to any far field microscopy method; compared with [Burns et al. 1983], the SPDM approach was explicitly based on the independent registration of the diffraction patterns of individual point sources; compared with the GSD concept [Hell and Kroug 1995] it considered the optical isolation of individual point sources by a variety of 'spectral signatures' as well as the extension to homogeneous illumination modes. In particular, [Cremer et al. 1996, 1999; Bornfleth et al. 1998; Edelmann 1999] described in detail the use of differences in the fluorescence emission spectrum and methods to perform the precise multispectral calibration measurements required to account for the correction of chromatic aberrations, as well as algorithms for subvoxel precision positioning in 2D (object plane) and in 3D (also along the optical axis), including the influence of the photon statistics. Even in optimally corrected far field microscopes, chromatic aberrations were found to be in the order of several tens of $\mathrm{nm}$ in the lateral direction and up to $200 \mathrm{~nm}$ in axial direction, depending on the conditions used [Esa et al. 2000; Rauch et al. 2000]; hence, without such calibrations a resolution enhancement in the sense of object position and distance measurements far beyond the Abbe-limit would not give reliable spatial information. Since the calibration errors are substantially lower in the lateral direction (object plane), [Cremer et al. 1996] previously discussed approaches to combine epifluorescence microscopy based localization microscopy with micro-axial tomography (section 5.3); in addition, they discussed a combination with a specific mode of structured illumination microscopy to realize a localization precision down to the $1 \mathrm{~nm}$ range [Albrecht et al. 2001, 2002] (section 5.2). To make possible the detection of multiple point sources within an Airy disk, in addition to differences in the fluorescence emission spectrum they proposed the use of differences in fluorescence lifetime, in luminescence, or in any other optical characteristics ("spectral signature") that are useful for optical isolation (i.e. the independent registration of the diffraction patterns of the individual point sources). This included the explicit introduction of the time domain: in these cases the enhanced 
resolution was not obtained by scanning in space (as in the previous nanoscopy approaches) but by 'scanning in time.' It may be noted that the word "spectral" by the authors in the "spectral signature" concept was not restricted to differences in the absorption/emission spectrum but was used according to its general meaning (see e.g. Webster's Dictionary) to denote any distribution of physical characteristics, including the time domain. However, in these papers the authors did not explicitly mention the possibility to use photoswitching ("blinking") of single molecules/point emitters for their localization microscopy approach.

In the following years, the SPDM approach of localization microscopy as well as related approaches by other groups were worked out further [Bornfleth 1998; Cremer et al. 1999, 2002; Lacoste et al. 2000; Schmidt et al. 2000; Heilemann et al. 2002], and first 'proof-of-principle' experiments were performed to indicate its feasibility. These experiments were based on precision position / distance measurements of objects optically isolated from each other by appropriate spectral signatures, such as fluorescence emission differences, or differences in the fluorescence life times of single, closely adjacent molecules. Distances in 2D/3D down to about 30 to $50 \mathrm{~nm}$ were measured, depending on the details of the method used [Cremer et al. 1996; 1999; Esa et al. 2000; 2001; Heilemann et al. 2002].

In the case of localization microscopy, the definition of the optical resolution (the smallest resolvable distance between two fluorescent point emitters) has been proposed to relate to the localization accuracy of the individual point sources [van Aert et al. 2006]; the estimate of the attainable structural resolution has to take into account also the density of optically isolated point emitters, depending on the peculiarities of the structures to be resolved [Lemmer et al. 2008; Kaufmann et al. 2009; Cremer et al. 2010]. With this method, cellular and other biological nanostructures labelled with fluorescent markers have been analysed down to the molecular optical resolution range (using the criterion of localization accuracy).

Since in an object to be studied, all point emitters of a given spectral signature can be resolved which have a mutual distance larger than the conventional resolution, already two or three spectral signatures allow highly relevant applications to biological nanostructure analysis [Cremer et al. 1999; Esa et al. 2000; 2001; Rauch et al. 2008; Hüve et al. 2008; Tykocinski et al. 2010]. It was evident, however, that the higher the number of spectral signatures, the better small structures can be analyzed. The use of fluorescence life times provided the possibility to increase the number of useful spectral signatures. Assuming 5 to 7 spectral signatures based on differences in the fluorescence absorption and on the emission spectrum (at $\sim 300 \mathrm{~K}$ ) and a few additional spectral signatures based on fluorescence life times of the $\mathrm{S}_{1}$ state, approximately 10 spectral signatures would already have been useful for the SPDM/localization microscopy techniques described so far to obtain valuable nanostructural information [Cremer et al. 1999]. In other types of fluorescence based optical analysis, ten and more absorption/emission based spectral signatures have become routine [e.g. Herzenberg et al. 2002]; in a localization microscopy approach based on such signatures, this would translate to a limit of up to several hundred distinguishable targets per $\mu \mathrm{m}^{2}$ (corresponding to a severalfold enhancement of resolution according to the Nyquist theorem).

\section{Localization microscopy techniques based on 'blinking'}

Although the early 'proof-of-principle' experiments mentioned were performed by using either scanning devices (confocal/near field optical scanning), and/or low temperature conditions, the application of the localization microscopy concept to enhanced resolution using farfield light microscopy in a homogeneous illumination mode and in the $300 \mathrm{~K}$ temperature range was explicitly recognized [Cremer et al. 1996, 1999]. 
In addition, ideas to use localization microscopy to obtain high resolution images by stochastic optical reconstruction schemes using random labeling procedures were also acknowledged [Cremer et al. 2002]. However, the special conditions considered in these early concepts were restricted to the use of a mixture of differentially labeled molecules, greatly limiting the number of molecules of the same type to be localized within a given Airy disk.

In the following years, various groups developed improved methods of localization microscopy to allow enhanced resolution by stochastic optical reconstruction (see below). These methods work even in the case that the molecules to be resolved are of the same type and labeled with the same fluorophore.

To our knowledge, the first experiments to use a point source induced to emit short fluorescent flashes distributed stochastically in time to realize high resolution localization microscopy using the same type of point emitters have been described by the Rainer Heintzmann group at King's College London [Lidke et al. 2005]. The intermittent fluorescence or 'blinking' of quantum dots was registered and analysed using independent component analysis so as to identify the light emitted by each individual nanoparticle, and to localize it precisely, and thereby resolve groups of closely spaced $(d<\lambda / 30)$ quantum dots. It became difficult, however, to reliably separate in the way described more than five emitters within a region of $200 \mathrm{~nm}$ diameter. Another setback for a wide spread application of this 'blinking' approach was the use of nanoparticles as fluorescent emitters. The authors stated, however, that this should be taken as a limitation of the present approach and not of the blinking-based separation procedure in general.

Within recent years, localization microscopy approaches using one or another scheme of "optical isolation" have been developed to allow a broad spectrum of applications (for reviews see [Zhuang 2009; Cremer et al. 2010, 2011; Cremer 2012]). Presently, they allow the localization of several to many thousand individual molecular signals $/ \mu \mathrm{m}^{2}$, corresponding to about 60 molecules in an area as small as an individual Airy disc.

Since 2006, various homogeneous, spatially non-scanning illumination schemes for photoactivation and/or photoswitching induced reversible or irreversible transitions of organic fluorochromes between optically distinguishable molecular states A and B have been used to achieve enhanced resolution. In one type of approaches, one wavelength is for photoactivation and/or photoswitching and another wavelength is for fluorescence excitation of the molecules to register the molecule positions; in a second type of approaches, one wavelength only is used for a given type of molecules, in combination with appropriate physico-chemical conditions; it is even possible to use one laser frequency only for two given molecule types simultaneously. The required optically distinguishable molecular states A and B (also called 'dark' and 'bright') [Hell 2009] do not necessarily mean emission and or nonemission of photons, but they may refer to the registration status of the detector for a particular spectral signature, including the time domain. Various methods using this principle for single molecule enhanced resolution have been realized during the last few years and successfully applied to a variety of biological nanostructures, from bacteria to tissue sections.

These techniques presently include the following: PALM (PhotoActivated Localization Microscopy) [Betzig et al. 2006, Biteen et al. 2008; Matsuda et al. 2010; Sengupta et al. 2011]; FPALM (Fluorescence Photoactivable Localization Microscopy) [Hess et al. 2006, 2007; Gould et al. 2008]; STochastic Optical Reconstruction Microscopy (STORM) [Rust et al. 2006; Bates et al. 2007; Huang et al. 2008]; PALM with Running Acquisition (PALMIRA) [Bock et al. 2007; Egner et al. 2007; Geisler et al. 2007]; Spectral Precision Distance Microscopy (SPDM) using reversibly photobleached single molecule states (also denominated as SPDM $_{\text {Phymod }}$ [Reymann et al. 2008; Lemmer et al. 2008, 2009; Kaufmann et al. 2009, 2011a, 2012; 

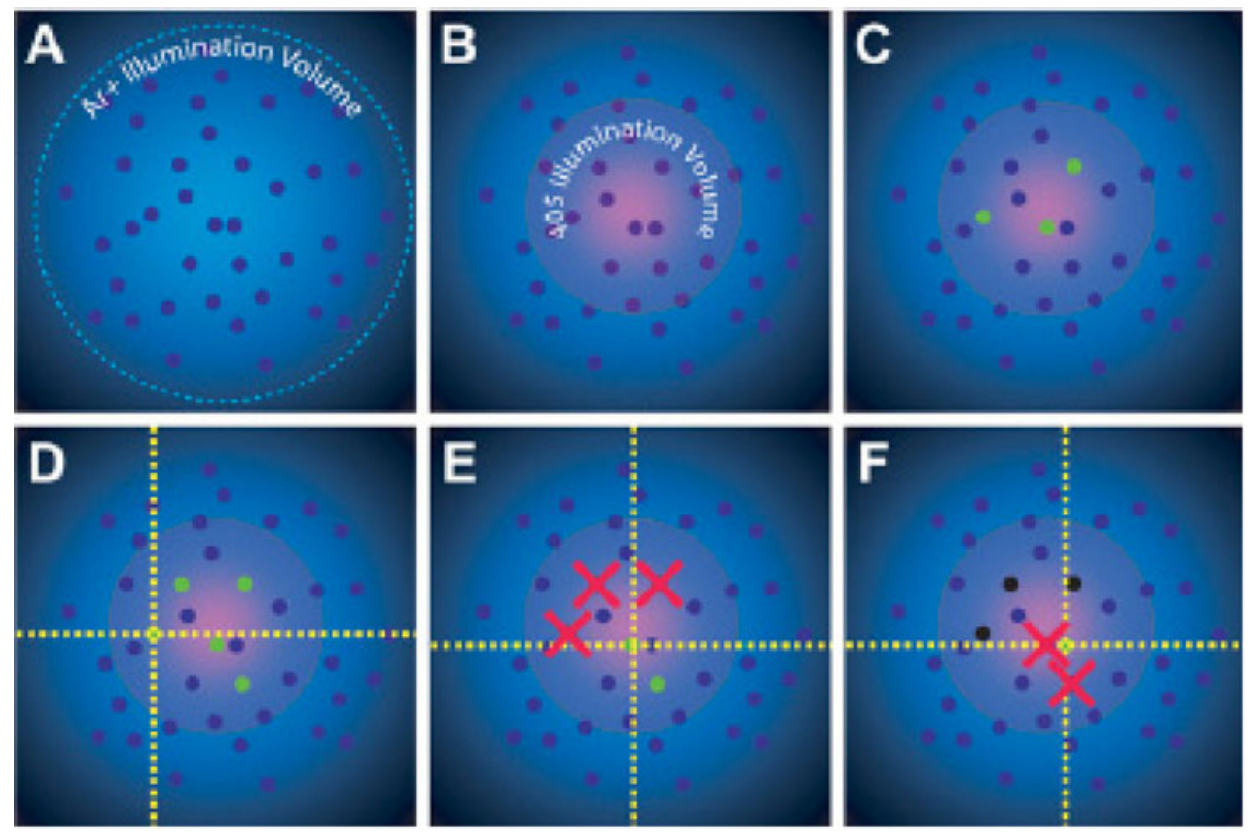

Fig. 5. Principle of Localization Microscopy based on photo-activable Green Fluorescence Proteins (PA-GFP). An area containing photoactivatable molecules (here, PA-GFP) is illuminated simultaneously with two frequencies of light, one for readout (here, an Ar ion laser with principal wavelengths $476.5 \mathrm{~nm}$ and $496.5 \mathrm{~nm}$, its spatial illumination profile shown in A), and a second one for activation (here, a 405-nm diode laser, its profile superimposed in B). Within the region illuminated by the activation beam, inactive PA-GFPs (small dark blue circles) are activated (C) by low intensity illumination at $405 \mathrm{~nm}$ (small green circles) and then localized (D) by using excitation with the $488 \mathrm{~nm}$ readout laser. After some time, the active PA-GFPs (E) photobleach (red Xs) and (F) become irreversibly dark (black circles). Additional molecules are then activated, localized, and bleached until a sufficient number of molecules have been analyzed to construct an image. From [Hess et al. 2006], with kind permission from the Biophysical Society.

Cremer et al. 2010, 2011; Kaufmann et al. 2012; Cremer 2012]; direct STORM (dSTORM) [Heilemann et al. 2008; Steinhauer et al. 2008]; Ground State Depletion Imaging Microscopy (GSDIM) [Fölling et al. 2008]; Dual Color Localization Microscopy (2CLM) [Gunkel et al. 2009]; Reversible Photobleaching Microscopy (RPM) [Baddeley et al. 2009a]; or 4D STORM (d ${ }^{4}$ STORM) [Baddeley et al. 2011].

For example, in the (F)PALM approach a UV laser beam is used at very low illumination intensities to induce sparsely distributed conformational changes in photoactivatable Green Fluorescent Proteins (PA-GFP), leading to a change in the fluorescence emission spectrum ('activation') and producing a 'bright' state. Here the basic idea has been to activate only a very few of the fluorophores, to have not more than one of these per diffraction volume. The fluorescence emission of the individual, optically isolated molecules is registered until they are bleached (converted into an irreversibly 'dark' state). After this first cycle all fluorophores are again in the 'dark' state. Now a new subset of the PA-GFP molecules is activated, detected and bleached. This procedure is repeated many times to detect a large number of single molecules (Figure 5).

A great advantage of this method has been its use in live cell time lapse microscopy [Hess et al. 2007]; however, it requires specially designed variants of fluorescent proteins. STORM can also be used for live cell imaging [Jones et al. 2011]. 
For many applications, it remained desirable to develop analogous photoswitching methods for use with conventional fluorochromes. Such a method has first been described as STORM [Rust et al. 2006]. STORM, a localization microscopy approach using photoswitchable probes, such as cyanine dyes, was first described as a method that uses pairs of standard organic fluorochromes and two frequency switching to enhance the resolution using one type of standard fluorochrome. It may be noted, however, that the inventors of STORM stated that this method should be applicable to any other type of suitably photoswitching molecule, and they noted that "the STORM concept is also applicable to other photoswitchable fluorophores and fluorescent proteins...".

Another way to experimentally realize the appropriate conversion between states A ('dark') and states B ('bright') under homogeneous illumination conditions (with respect to the region of interest) without needing specially designed molecules or pairs of molecules which have to be photoactivated or photoswitched by two different wavelengths has been described by [Bock et al. 2007; Reymann et al. 2008; Lemmer et al. 2008, 2009; Heilemann 2008; Steinhauer et al. 2008; Zhuang et al. 2009]. This approach takes advantage of the fact that under certain illumination conditions combined with specific physicochemical conditions, a rapid switching may occur between bright and dark states [Bock et al. 2007; see below]; or in many types of fluorescent molecules two types of 'dark' states may be induced using one wavelength alone [Reymann et al. 2008; Lemmer et al. 2008, 2009]: one irreversibly bleached state $\mathrm{M}_{\text {irr }}$ and one reversibly bleached state $\mathrm{M}_{\mathrm{rb}}$ [Sinnecker et al. 2005]. After excitation with a suitable single wavelength and illumination intensity, they can either pass to the irreversibly bleached 'dark' state $\mathrm{M}_{\mathrm{irr}}$ or they can pass to the reversibly bleached 'dark' state $\mathrm{M}_{\mathrm{rb}}$. From this 'dark' state they can stochastically re-enter the fluorescent state $\mathrm{M}_{\mathrm{fl}}$ in which they emit in a few tens of milliseconds a flash of thousands of photons before they pass into either the irreversibly bleached 'dark' state $\mathrm{M}_{\mathrm{irr}}$, or are again transferred to the reversibly bleached state $M_{r b}$. At appropriately long time constants for the transitions (seconds to minutes) of the fluorophores from the reversibly bleached state $M_{r b}$ to the fluorescent state $M_{f l}$ the distances between the diffraction images of the molecules are large enough to allow the desired optical isolation even with relatively slow CCD cameras. Thus the positions of the individual detected fluorophores can be determined according to the basic principles of localization microscopy outlined above. In this case, the spectral signature (according to the general definition of this term given e.g. in [Cremer et al. 1996, 1999, 2002] is the transition time of the individual molecule from the reversibly bleached state $\mathrm{M}_{\mathrm{rb}}$ to the fluorescent state $\mathrm{M}_{\mathrm{fl}}$. Interestingly, this transition was observed to be induced by using only a single laser frequency at constant illumination intensity (in space and time) for both the induction of long (up to the hundred second range) reversible bleaching transitions and fluorescence registration [Reymann et al. 2008; Lemmer et al. 2008, 2009]. Since this enhanced resolution technique is based on the general principles of SPDM using molecular states modified by appropriate illumination intensities and environmental conditions, it was denominated as SPDM with physically modifiable fluorophores $\left(\mathrm{SPDM}_{\text {Phymod }}\right.$ ) [Kaufmann et al. 2009; Lemmer et al. 2009]. Typically, a few thousand frames registered at a frame rate of $20 \mathrm{fps}$ or more are sufficient to render an image with a presently achieved resolution down to the $10 \mathrm{~nm}$ range (as estimated from the localization accuracy). Instead of using relatively high illumination intensities, Bock et al. 2007 have shown that standard fluorophores (Cy5) can be applied for localization microscopy at relatively low illumination intensities $\left(10 \mathrm{~kW} / \mathrm{cm}^{2}\right.$ $633 \mathrm{~nm}$ excitation) using the same laser frequency for photoswitching and readout.

In this case, however, switched-off Cy5 molecules thermally recovered within $100 \mathrm{~ms}$ to their bright on-state, and registration times in the 2 millisecond range were used; in the $\mathrm{SPDM}_{\text {Phymod }}$ mode mentioned above, switched-off molecules 
(e.g. standard synthetic dyes like Alexa 488, 568, or conventional fluorescent proteins like eGFP, YFP, or mRFP) on the average recovered after minutes only, and typical registration times around 50 Milliseconds were applied. Another difference was the use of a standard embedding medium or even phosphate based saline (PBS) in $\mathrm{SPDM}_{\text {Phymod, }}$, allowing the use of this mode of localization microscopy even under live cell conditions.

An especially intriguing consequence of the $\mathrm{SPDM}_{\text {Phymod }}$ approach and other localization microscopy methods like 2CLM, RPM, dSTORM, d ${ }^{4}$ STORM, or GSDIM has been the possibility to use standard fluorescent dyes as well as conventional fluorescent proteins in a microscope setup even at homogeneous illumination conditions (with respect to the field of view chosen). Since such "standard" fluorophors had already been introduced into a vast spectrum of conventional fluorescence microscopy applications, this technique has become particularly useful for a broad spectrum of applications in the biosciences. Theoretical considerations [Cremer et al. 2010] indicated that at the registration speeds presently realized, the average dark (reversibly bleached) to bright (fluorescent state) transition times have to be in the order of many seconds to allow the desired optical isolation at relatively slow registration rates (e.g. 20 frames/s): i.e. two adjacent molecules have to emit their photon flashes at sufficiently different times so that the diffraction patterns produced by these photon emissions can be distinguished from each other and thus used for the localization of the individual molecules.

\section{Relationships between various localization microscopy techniques}

Comparing the various single-molecule high-resolution imaging approaches using standard fluorophores (i.e. fluorochromes commonly used in conventional fluorescence imaging) outlined above, various similarities but also major differences may be noted. For example, in the technique of Single-molecule High-Resolution Imaging with Photobleaching (SHRImP), [Gordon et al. 2004] localized pairs of single Cy3 molecules with $\mathrm{nm}$ precision and determined their separation with $5 \mathrm{~nm}$ precision, using their quantal photobleaching behaviour. However, according to the authors this method did not allow true imaging because it could only be used to measure distances with well separated pairs of molecules, not multiple molecule positions within the conventional resolution limit like in the other localization microscopy techniques mentioned, like PALM, FPALM, STORM, dSTORM, GSDIM, SPDM Phymod $_{\text {etc. }}$

This restriction probably is due to the fundamental difference how the photobleaching behaviour of single molecules was used. While in SHRImP, the basic photophysical effect used was the stepwise, bleaching induced decrease in the total fluorescence intensity measured from a pair of adjacent dye molecules, the above mentioned single molecule localization microscopy approaches allow the localization of an arbitrary high number of molecule signals within the conventional resolution limit; this is possible as long as the basic conditions of all localization microscopy methods is maintained, the optical isolation due to an appropriate (in this case time dependent) spectral signature.

Although the technical requirements (high mechanical stability, sensitive CCD cameras (or related devices) to detect single standard molecule fluorescence emission) for these highly simplified and robust modes of localization microscopy on the basis of homogeneous illumination schemes have existed at least for a decade, accounts of their realization for a variety of conditions, targets and applications have been published only recently, within a few months and independently from each other by a variety of groups. For example, [Bock et al. 2007] (manuscript received June 20 and published online 6 July 2007) used the organic fluorphore Cy5 in combination with $633 \mathrm{~nm}$ excitation to perform PALMIRA based localization microscopy of the 
images of the microtubular network in a PtK2 cell; [Reymann et al. 2008] (revised manuscript received February 21 and published 8 May 2008) described enhanced resolution of nuclear pore complexes using the standard dye Alexa488 (homogeneous illumination at $\lambda_{\text {exc }}=488 \mathrm{~nm}$ ) in combination with a standard embedding medium, to be followed shortly afterwards by [Heilemann et al. 2008] (received 21 May 2008 and published 22 July 2008) and [Steinhauer et al. 2008] (received 19 August and published 24 November 2008) describing the use of Cy5/Alexa 647 in combination with certain specially made 'switching buffers' to perform enhanced resolution of DNA and cellular Actin filaments; [Lemmer et al. 2008] (received 8 June and published 1 September 2008; US patent application Lemmer et al. filed March 2008, granted March 2012) described the extension of $\mathrm{SPDM}_{\text {Phymod }}$ to standard Fluorescent Proteins (YFP), achieving a lateral optical resolution down to the 10 to $20 \mathrm{~nm}$ regime; in combination with SMI-microscopy, three dimensional images were obtained corresponding to a 3D-resolution of thin cellular structures (axial thickness $<150 \mathrm{~nm}$ ) of 40 to $50 \mathrm{~nm}$ $\left(\sim 1 / 10\right.$ of $\left.\lambda_{\text {exc }}\right)$. [Lemmer et al. 2008; Kaufmann et al. 2011a].

Another early report of localization microscopy using homogeneous illumination in combination with standard fluorochromes called Ground State Depletion Imaging Microscopy (GSDIM) was published by [Fölling et al. 2008] (received 24 July and published 15 September 2008), featuring an enhanced lateral resolution of microtubules and peroxisomes immunostained with standard fluorophores Atto 532/565 in the $<30 \mathrm{~nm}$ range. In this latter case, the term scheme $\left(\mathrm{S}_{0}\right.$ to $\mathrm{S}_{1}$ to $\mathrm{T}_{1}$ to $\left.\mathrm{S}_{0}\right)$ presented previously for relatively fast reversible transitions between dark and bright states ( $\mu$ s to $\mathrm{ms}$ ) in GSD microscopy was extended by Fölling et al. to include very long lived dark state $\mathrm{D}$ (transition times $\mathrm{D}$ to $\mathrm{S}_{0}$ in the required order of seconds, i.e. several orders of magnitudes higher that the $T_{1}$ to $S_{0}$ transitions of GSD microscopy). This state $D$ was conceived to be accessible via a $S_{0}$ to $S_{1}$ to $T_{1}$ transition.

From the formal point of view this general scheme appears to be compatible with mechanisms proposed previously for reversible photobleaching [Sinnecker et al. 2005; Lemmer et al. 2008]. Compared to the dSTORM concept as published originally [Heilemann et al. 2008], major differences between SPDM Phymod $_{\text {and dSTORM are }}$ the molecule types and biostructures studied, the chemical environment used, as well as the two laser excitation schemes used originally by [Heilemann et al. 2008].

The various denominations given to the novel localization microscopy approaches which made it possible to include even standard fluorochomes in a straightforward way are justified by the many differences in the optical setup, the molecule types, physicochemical environments used. In addition, the different abbreviations stress various elements of the entire localization microscopy concept. For example, in the acronyms "PALM" and "FPALM" the importance of using photoswitchable molecules is put forward. In "STORM", "dSTORM" and "d ${ }^{4}$ STORM" the optical isolation by stochastically distributed spectral signatures is highlighted. In the acronym GSDIM the focus is put on the quantum physical necessity to realize very long lived excited states for $\mathrm{S}_{0}$ depletion. In "RPM" the effect of reversible photobleaching is denoted. In 2CLM the possibility to extend localization microscopy to the simultaneous enhanced resolution of multiple molecule types is envisaged. And in $\mathrm{SPDM}_{\text {Phymod, }}$, the aspect to perform highly precise position and distance measurements of single molecules under specific physicochemical conditions (e.g. illumination intensity, chemical environment) is regarded [Cremer et al., 2010, 2011; Cremer 2012]. It may be noted that many details of the underlying physicochemical and photophysical mechanisms are still poorly understood. Recent experimental evidence indicates that spatial configuration changes are involved not only in the photoswitching of photoactivable fluorescent proteins [Dickson et al. 1997] but also in the photoswitching of standard fluorescent proteins [Matsuda et al. 2010]. 


\section{Combination of localization and structured excitation illumination microscopy}

First attempts have been reported to combine localization microscopy with confocal microscopy and or structured illumination schemes; this may be highly advantageous to obtain such an enhanced resolution not only in the object plane but even in three dimensions.

For example, already a decade ago the combination of a localization microscopy approach (SPDM) with confocal laser scanning fluorescence microscopy allowed 3D distance measurements down to $50 \mathrm{~nm}$ range [Esa et al. 2000]. Although this attempt was restricted to the optical isolation of a few closely adjacent fluorescent point emitters only, it was sufficient to obtain spatial information about chromatin nanostructure in cell nuclei of cancer patients not available by conventional resolution. Recently, the combination with 4Pi microscopy [Hüve et al. 2008] permitted 3D distance measurements of intracellular targets with a localization accuracy around $10 \mathrm{~nm}$, corresponding to an enhanced resolution around $20 \mathrm{~nm}$. The combination of SPDM Phymod and Spatially Modulated Illumination (SMI) microscopy allowed to generate images of (thin) biological structures corresponding to the results achievable with a $3 \mathrm{D}$ effective optical resolution in the 30 to $50 \mathrm{~nm}$ range [Lemmer et al. 2008; Kaufmann et al. 2011a]. Numerical simulations [Failla and Cremer 2001; Albrecht et al. 2001] suggested that in the case of detected photon counts in the $10^{3}$ to $10^{4}$ range, even a $3 \mathrm{D}$ optical resolution in the $1 \mathrm{~nm}$ range eventually might become feasible for single molecule based localization microscopy.

\section{Concluding remarks}

\subsection{The enhancement of resolution in time and space and its potential applications}

In this historical survey an attempt was made to delineate basic milestones in the development of resolution enhancement techniques. A particular goal was to summarize the many lines of developments to overcome the hundred year old limitations in far-field light microscopy. The possibilities to 'break', or more correctly, to 'circumvent' the limits imposed by Abbe's and Rayleigh's theories have become so many that it might be helpful to find a joint name. Presently, terms like 'enhanced resolution light microscopy,' 'light-optical nanoscopy', 'superresolution optical microscopy,' or (in the Anglo-American tradition to give well memorisable names) even 'LOBSTER' (Light-Optical BioStructure analysis at Enhanced Resolution) have been proposed [Cremer et al. 2011; Cremer 2012]. To avoid the ambiguous term 'LM' (which stands for Light Microscopy in general) as an abbreviation for the now well accepted general term 'localization microscopy', acronyms such as SMLM (Single Molecule Localization Microscopy) [McEvoy et al. 2010] or SALM (Spectrally Assigned Localization Microscopy) [Markaki et al. 2010; Cremer 2012] have been proposed.

While in 'main stream' physics, the resolution limits of far-field light microscopy were thought to be once for all settled and thus not interesting any more, the pioneer groups in this field mostly consisted of physicists connected to a biological scientific environment where the gain of microscopic resolution was a constant challenge. As previously noted many advances in enhanced resolution microscopy were also made in the fields of lithography and nanofabrication in addition to those made in the biological fields.

In the aftermath, at least some of the optical principles leading to "enhanced resolution' might have been formulated already many decades before this was actually done. For example, at the time that Lord Rayleigh formulated his theory it was 
known that the maximum of the Airy disc corresponded to the position of a point object. Perhaps it was regarded to be too trivial to explicitly draw the conclusion that by changing the fluorescent wavelength or some other discriminating spectral characteristics, objects with a distance much smaller than half the wavelength could be independently localized from each other, and hence even subresolution distances determined. However, once that the psychological barrier had been broken by the demonstration that indeed an enhanced resolution below the "Abbe limit" was possible, the further development to the present state of far-field enhanced resolution microscopy (lateral two point resolution in the $1 \mathrm{~nm}$ range achieved for two adjacent molecules of the same type, corresponding to one five hundredth of the exciting wavelength [Pertsinidis et al. 2010]) took less than two decades.

Perhaps the first experimental proof that a substantial enhancement of optical resolution was possible in farfield microscopy at least in the axial direction was obtained in the first half of the 1990s by Stefan Hell and his colleagues [Hell and Stelzer 1992a,b; Hell et al. 1994a,b; Lindek et al. 1994; Hänninen et al. 1995] using 4Pi confocal microscopy with two opposing high $N A$ lenses. This first breakthrough was soon followed by the development of the concepts of STED microscopy [Hell and Wichmann 1994; Baer 1994] and of Ground State Depletion based focused nanoscopy [Hell and Kroug 1995]. About the same time, the first concepts of multitarget localization microscopy have been put forward [Betzig et al. 1995; Cremer et al. 1996, 1999; van Ojen et al. 1998; Bornfleth et al. 1998].

\subsection{Impact of enhanced resolution light microscopy in the biosciences}

Presently, enhanced resolution light microscopy techniques have already found a large field of applications. For example, 4Pi-microscopy and STED-microscopy have been used to study nanostructures on the cell membrane [Bahlmann et al. 2001; Glaschick et al. 2007; Perinetti et al. 2009], of the cytoplasm [Egner et al. 1998; Hell and Nagorni 1998; Nagorni and Hell 1998; Schrader et al. 1998; Gugel et al. 2004; Egner et al. 2005] as well of the nucleus [Bewersdorf et al. 2006; Lang et al. 2010] and of the nuclear envelope [Hüve et al. 2008; Baddeley et al. 2006]. In addition, STED microscopy has been shown to be useful for neurobiology in the study of synaptic connections between nerve cells [Willig et al. 2006; Nagerl et al. 2008]. Structured illumination microscopy approaches are presently used for a similar range of applications [Martin et al. 2004; Hildenbrand et al. 2005; Birk et al. 2007; Schermelleh et al. 2008; Reymann et al. 2008; Baddeley et al. 2010b; Markakis et al. 2010]; in addition, they have been shown to allow an improved imaging quality of retina tissue, e.g. to analyze age dependent macula degeneration [Best et al. 2011; Ach et al. 2012]. Localization microscopy applications are fast extending their application potential; presently, they range from the molecular resolution of membrane structures in normal and cancer cells, the quantitative single molecule arrangement in connections between nerve cells and cytoplasmic multiprotein fibers to various nanostructures in the cell nucleus, including the machines for DNA transcription, repair, and gene regulation, to the detection of individual HIV viruses and even the shape of small viruses (so far reserved to electron microscopy) [Esa et al. 2000, 2001; Rauch et al. 2000, 2008; Betzig et al. 2006; Hess et al. 2006, 2007; Bock et al. 2007; Biteen et al. 2008; Reymann et al. 2008; Lemmer et al. 2008, 2009; Shroff et al. 2008; Gunkel et al. 2009; Markakis et al. 2010; Tykocinski et al. 2010; Baddeley et al. 2009a,b, 2011; Kaufmann et al. 2009, 2011a,b, 2012a,b; Huber et al. 2012; Wombacher et al. 2010; Klein et al. 2011; Huber et al. 2012; Löschberger et al. 2012; Pereira et al. 2012; Ries et al. 2012; for reviews see Zhuang 2009; Cremer et al. 2010, 2011; van de Linde 2011]. 
While all these applications demonstrate the usefulness of enhanced light microscopy methods, its potential to trigger a similar revolution of biology and medicine as the improvement of microscopy in the nineteenth century still appears not to be evident.

The historical evidence accumulated for thousands of years in astronomy and since the 1600s in physics and other sciences demonstrates that progress in methods of quantitative measurement is intimately connected with the progress in scientific insight. The experience of microscopy indicates an analogous connection: the enhancement in usable resolution and contrast made possible since the invention of the first microscopes in the 1600s and the progress in the biosciences clearly correlate. Modern biology and medicine is enhanced by being able to visualize the cellular structure of organisms, to detect bacteria, and to analyze individual cells in a tissue. From this historical experience, one would expect that the enhancement of light-optical resolution and contrast achieved within the last decades should have a similar impact on the future of biosciences.

In the 1600s and 1700s, the use of the microscope in the biosciences was hampered by technical difficulties (such as strong chromatic and monochromatic aberrations, low illumination intensities, lack of appropriate specimen preparation). An additional reason might have been the leading medical paradigm of the importance of bodily humors (blood, phlegm, choler, black bile) regarded to determine the general health of a person. This attitude to disregard the importance of the spatial microstructure was radically overcome with the microscopy based development of modern cellular theory, cellular pathology, and microbiology.

Today, the introduction of enhanced light microscopy methods into the biosciences appears to be slowed down by other reasons: the advent of electron microscopy in the 1930s and of X-ray crystallography of biostructures in the 1950s have opened a broad avenue to study cellular nanostructures down to the atomic resolution level of many of their constituents; in addition, the progress of biochemistry and of molecular biology has opened an avenue to know all the molecular components of a cell and their average interactions. In many molecular biology research groups, the only microscope is a small phase contrast system for cell culture; nonetheless, these groups are capable of deciphering the average cellular interactions down to the molecular scale. The combination of molecular biology with high resolution electron microscopy and X-ray crystallography allows them to obtain quite precise ideas of nanostructural interactions on a scale which does appear to make obsolete even be most recent progress in enhanced resolution light microscopy.

On the other side, methods of biochemistry and molecular biology typically work with results obtained not from a single cell but of many cells; they produce average values. However, the single cell may matter; in developmental biology, a single stem cell may be the ancestor of a line of specialized cells (e.g. of a tissue) or even an entire organism; in medicine, a single metastatically competent cell may result in the death of the individual; or a single pluripotent stem cell may reconstitute a cell line allowing to live; a few single viruses interacting successfully with the cell membrane may lead to a HIV infection; the distribution of single drug molecules in a tissue or at the blood-brain barrier may have severe physiological consequences.

These examples indicate that enhanced light microscopy methods are likely to have a major impact in the biosciences. This will be especially true if they can be further developed in a way to allow fast 'high-throughput' analyses (e.g. obtain multicolor localization microcopy images of 1000 cells with $1 \times 10^{12}$ molecule positions assigned in one day). To what extent these promising application perspectives will lead not only to major impacts in medicine but also produce completely new insights in biology remains to be seen. 
In all the pioneer groups involved there was a strong conviction that the limits stated by Abbe and Rayleigh were not directly "breakable" (in the sense that Abbe and Rayleigh were wrong) but might be circumvented by approaches not considered by these pioneers of optics more than 100 years ago (To highlight this very unexpected achievement, the term 'to break the Abbe limit' in the sense "to escape from, to surpass" often used in the literature may also be justified.) Furthermore, between many of these groups personal connections have existed for decades partially supported by joint grants.

To summarize, the development of resolution enhancing "nanoscopy" technologies is still in full development. It is to be expected that eventually each branch of the superresolution family (focused/structured/homogeneous illumination based nanoscopy) will produce highly vigorous descendants, adapted to their special niches, i.e. biomedical and material sciences application fields.

It should be stressed that optical aberrations will reduce the resolution as compared to an aberration free optical system. The effects of high power laser radiation on live cells, tissues and organisms, as well as photobleaching and photodamage are compelling. The use of fluorescent probes, as well as the overexpression of genetically encoded fluorescent proteins can affect the biological function of cells and tissues. The use of these techniques with thick, highly scattering specimens is very challenging, especially with techniques that depend on structured illumination, and STED techniques, where the desired, theoretical structure of the light distribution may differ considerably from the actual structure within the specimen. Finally, the cost of pulsed laser sources and problems of alignment may hinder the wide spread use of some of these resolution enhancing techniques. The experience gained from the history of science suggests, however, that many of these problems shall be overcome, due to the importance of microscopy for almost all fields of biology and medicine.

\section{Websites}

Instead of giving many examples of the use of enhanced resolution microscopy in cell biology and medicine we chose to present several websites with various applications. The authors have selected a few of the interesting websites on the basis of their tutorial value. We apologize to the many other research groups for the fact that we are unable to cite all of the relevant websites.

Betzig, E. http://www.janelia.org/lab/betzig-lab

Cremer, C. http://www.kip.uni-heidelberg.de/AG_Cremer; http://www.imb-mainz. de/research-at-imb/Cremer

Hell, S. http://www.4pi.de/

Hess, S. http://www.physics.umaine.edu/FPALM_SFS_NOV09/fpalm_principle.html Sauer, M. http://www.super-resolution.biozentrum.uni-wuerzburg.de/en/home/ dstorm/

Sedat, J. http://msg.ucsf.edu/sedat/

Zhuang, X. http://zhuang.harvard.edu/index.html

Acknowledgements. We thank the anonymous reviewers for their helpful comments. We thank the editor Prof. Wolf Beiglboeck also for his critical suggestions. The citations made are intended to permit further reading; we apologize to have not been able to quote all relevant papers from a now exponentially growing literature. The presentation of Section 5 is partly based on previous reviews by one of the authors [Cremer 2010, 2011; Cremer et al. 2011; Cremer 2012]. 


\section{References}

Abbe, E. 1873. Beiträge zur Theorie des Mikroskops und der mikroskopischen Wahrnehmung. M. Schultze's Archive für Mikroskopische Anatomie IX: 413-468

Abbe, E. 1989a. Abhandlungen über die Theorie des Mikroskops I, Goerg Olms Verlag, Hildescheim, Germany

Abbe, E. 1989b. Gesammelte Abhandlungen, four volumes, Goerg Olms Verlag, Hildesheim, Germany

Ach, T., G. Best, S. Rossberger, R. Heintzmann, C. Cremer and S. Dithmar. 2012. Autofluorescence imaging of human RPE cell granules using structured illumination microscopy, Br. J. Ophthalmology, DOI 10.1136/bjophthalmol-2012-301547

Agard, D.A. and J. Sedat. 1983. Three-dimensional architecture of a polytene nucleus. Nature 302: $676-681$

Airy, G.B. 1835. On the Diffraction of an Object-glass with Circular Aperture. Trans. Cambridge Philos. Soc. 5: 283-291

Albrecht, B.A., V. Failla, R. Heintzmann and C. Cremer. 2001. Spatially modulated illumination microscopy: online visualization of intensity distribution and prediction of nanometer precision of axial distance measurements by computer simulations. Journal of Biomedical Optics 6: 292-292

Albrecht, B.A., A. Failla, A. Schweitzer and C. Cremer. 2002. Spatially modulated illumination microscopy allows axial distance resolution in the nanometer range. Appl. Opt. 41: 80-87

Andresen, M., A.C. Stiel, F. Jonas, D. Wenzel, A. Schönle, A. Egner, C. Eggeling, S.W. Hell and S. Jakobs. 2008. Photoswitchable fluorescent proteins enable monochromatic multilabel imaging and dual color fluorescence nanoscopy. Nature Biotechnology 26: 10351040

Ash, E.A. and G. Nichols, 1972. Super-resolution aperture scanning microscope, Nature 237: 510-512

Baddeley, D., C. Carl and C. Cremer. 2006. 4Pi microscopy deconvolution with a variable point-spread function. Appl. Optics 45: 7056-7064

Baddeley, D., C. Batram, Y. Weiland, C. Cremer and U.J. Birk. 2007. Nanostructure analysis using spatially modulated illumination microscopy. Nature Protocols 2: 2640-2646

Baddeley, D., I.D. Jayasinghe, C. Cremer, M.B. Cannell and C. Soeller. 2009a. Light-induced dark states of organic fluorochromes enable $30 \mathrm{~nm}$ resolution imaging in standard media. Biophys. J. 96: L22-L24

Baddeley, D., I.D. Jayasinghe, L. Lam, S. Rossberger, M.B.Cannell and C. Soeller. 2009b. Optical single-channel resolution imaging of the ryanodine receptor distribution in rat cardiac myocytes. Proc. Natl. Acad. Sci. USA 106: 22275-22280

Baddeley, D., Y. Weiland, C. Batram, U. Birk and C. Cremer. 2010a. Model based precision structural measurements on barely resolved objects. J. of Microscopy 237: 70-78

Baddeley, D., V.O. Chagin, L. Schermelleh, S. Martin, A. Pombo, P.M. Carlton, A. Gahl, P. Domaing, U. Birk, H. Leonhardt, C. Cremer and M.C. Cardoso. 2010b. Measurement of replication structures at the nanometer scale using super-resolution light microscopy. Nucleic Acids Res 38: e8 1-11. doi:10.1093/nar/gkp901

Baddeley, D., D. Crossman, S. Rossberger, J.E. Cheyne, J.M. Montgomery, I.D. Jayasinghe, C. Cremer, M.B. Cannell and C. Soeller. 2011. 4D Super-Resolution Microscopy with Conventional Fluorophores and Single Wavelength Excitation in Optically Thick Cells and Tissues. PLoS ONE 6: e20645. doi:10.1371/journal.pone.0020645

Baer, S.C. "Method and Apparatus for improving resolution in scanned optical system", Filed: July 15, 1994, Date of Patent: February 2, 1999. U. S. Patent number: 5, 866, 911

Bahlmann, K., S. Jakobs and S.W. Hell. 2001. 4Pi-confocal microscopy of live cells. Ultramicroscopy 87: 155-164

Bailey, B., D. Farkas, D.L. Taylor and F. Lanni. 1993. Enhancement of axial resolution in fluorescence microscopy by standing-wave excitation. Nature 366: 44-48

Bates, M., B. Huang, G. Dempsey and X. Zhuang. 2007. Multicolor super-resolution imaging with photoswitchable fluorescent probes. Science 317: 1749-1753 
Best, G., R. Amberger, D. Baddeley, T. Ach, S. Dithmar, R. Heintzmann and C. Cremer. 2011. Structured illumination microscopy of autofluorescent aggregations in human tissue. Micron 42: 330-335

Bethe, H.A. 1944. Theory of diffraction by small holes. The Physical Review 66: 163-182

Betzig, E. 1995. Proposed method for molecular optical imaging. Optics Letters 20: 237-239

Betzig, E., A. Lewis, M. Isaacson, A. Murray and A. Harootunian. 1986. Near Field Scanning Optical Microscopy (NSOM): Development and Biophysical Applications. Biophysical Journal 49: 269-279

Betzig, E., G.H. Patterson, R. Sougrat, O.W. Lindwasser, S. Olenych, J.S. Bonifacino, M.W. Davidson, J. Lippincott-Schwartz and H.F. Hess. 2006. Imaging Intracellular Fluorescent Proteins at Nanometer Resolution. Science 313: 1642-1645

Bewersdorf, J., B.T. Bennett and K.L. Knight. 2006. H2AX chromatin structures and their response to DNA damage revealed by 4Pi microscopy. Proc. Natl. Acad. Sci. USA 103: $18137-18142$

Binnig, G. and H. Rohrer. 1982. Scanning tunneling microscopy. Helvetica Physica Acta 55: 726-735

Binnig, G., C.F. Quate and Ch. Gerber. 1986. Atomic force microscope, Physical Review Letters 56: 930-933

Birk, U.J., I. Upmann, D. Toomre, C. Wagner and C. Cremer. 2007. Size Estimation of Protein Clusters in the Nanometer Range by Using Spatially Modulated Illumination Microscopy, in: Modern Research and Educational Topics in Microscopy, edited by A. Mendez-Vilas, J. Diaz. FORMATEX Microscopy Series, Vol. 1, pp. 272-279

Biteen, J.S., M.A. Thompson, N.K. Tselentis, G.R. Bowman, L. Shapiro and W.E. Moerner. 2008. Single-moldecule active-control microscopy (SMACM) with photo-reactivable EYFP for imaging biophysical processes in live cells. Nature Methods 5: 947-949

Bock, H., C. Geisler, C.A. Wurm, C. von Middendorff, S. Jakobs, A. Schönle, A. Egner, S.W. Hell and C. Eggeling. 2007. Two-color far-field fluorescence nanoscopy based on photoswitchable emitters. Appl. Phys. B 88: 161-165

Bohn, M., P. Diesinger, R. Kaufmann, Y. Weiland, P. Müller, M. Gunkel, A. von Ketteler, P. Lemmer, M. Hausmann and C. Cremer. 2010. Localization Microscopy reveals expression dependent parameters of chromatin nanostructure. Biophys. J. 99: 1358-1367

Born, M. and E. Wolf. 1980. Principles of optics, Electromagnetic theory of propagation, interference and diffraction of light. 7th edn. (Expanded). Cambridge, Cambridge University Press

Bornfleth, H., K. Sätzler, R. Eils and C. Cremer. 1998. High precision distance measurements and volume-conserving segmentation of objects near and below the resolution limit in three-dimensional confocal fluorescence microscopy. J. Microscopy 189: 118-136

Bradl, J., M. Hausmann, V. Ehemann, D. Komitowski and C. Cremer. 1992. A tilting device for three-dimensional microscopy: application to in situ imaging of interphase cell nuclei. J. Microscopy 168: 47-57

Bradl, J., M. Hausmann, B. Schneider, B. Rinke and C. Cremer. 1994. A versatile 2pi-tilting device for fluorescence microscopes. J. Microscopy 176: 211-221

Bradl, J., B. Rinke, B. Schneider, M. Hausmann and C. Cremer. 1996a. Improved resolution in 'practical' light microscopy by means of a glass fibre 2pi-tilting device, in: Optical and Imaging Techniques for Biomonitoring, edited by Hans-Jochen Foth, Renato Marchesini, Halina Podbielska, Michel Robert-Nicoud, Herbert Schneckenburger, Proc. SPIE 2628: $140-146$

Bradl, J., B. Rinke, A. Esa, P. Edelmann, H. Krieger, B. Schneider, M. Hausmann and C. Cremer. 1996b. Comparative study of three-dimensional localization accuracy in conventional, confocal laser scanning and axial tomographic fluorescence light microscopy. Proc. SPIE 2926: 201-206

Brakenhoff, G.J., P. Blom and P. Barends. 1979. Confocal scanning light microscopy with high aperture immersion lenses. Journal of Microscopy 117: 219-232

Brakenhoff, G.J., H.T.M. van der Voort, E.A. van Spronsen, W.A.M. Linnemanns and N. Nanninga. 1985. Three-dimensional chromatin distribution in neuroblastoma nuclei shown by confocal laserscanning microscopy. Nature 317: 748-749 
Born, M. and E. Wolf. 1975. Principles of Optics, Pergamon, Oxford

Bretschneider, S., C. Eggeling and S.W. Hell. 2007. Breaking the diffraction barrier in fluorescence microscopy by optical shelving. Phys. Rev. Lett. 98: 218103-1-21803-4

Brunner, A., G. Best, P. Lemmer, R. Amberger, T. Ach, S. Dithmar, R. Heintzmann and C. Cremer. 2011. Fluorescence Microscopy with Structured Excitation Illumination, in: Handbook of Biomedical Optics, edited by D.A. Boas, C. Pitris and N. Ramanujam. CRC Press, Boca Raton

Burns, D.H., J.B. Callis, G.D. Christian and E.R. Davidson. 1985. Strategies for attaining enhanced resolution using spectroscopic data as constraints. Appl. Optics 24: 154-161

Chao, W., B.D. Harteneck, J.A. Liddle, E.H. Anderson and D.T. Attwood. 2005. Soft X-ray microscopy at a spatial resolution better than $15 \mathrm{~nm}$. Nature 435: 1210-1213

Chen, K.R. 2009. Focusing of light beyond the diffraction limit. arXiv.org/pdf/4623v1

Courjon, D. 2003. Near-Field Microscopy and Near-Field Optics, Imperial College Press, London

Cremer, C. 2011. Lichtmikroskopie unterhalb des Abbe-Limits. Physik in Unserer Zeit 42 : 21-29 (in German)

Cremer, C. 2011. Mikroskope und Mikroben. In: Viren und andere Mikroben: Heil oder Plage? Zum hundertsten Todestag von Robert Koch (Karlheinz Sonntag, Hg.). Studium Generale der Universität Heidelberg 2010. Universitätsverlag Winter, Heidelberg (in German)

Cremer, C. 2012. Optics far Beyond the Diffraction Limit From Focused Nanoscopy to Spectrally Assigned Localization Microscopy. Springer Handbook of Lasers and Optics, $2^{\text {nd }}$ edition (F. Träger, Editor, pp. 1351-1389 Springer Verlag, Berlin

Cremer, C. and T. Cremer. 1972. Procedure for the Imaging and modification of object details with dimensions below the range of visible wavelengths. German Patent Application No. 2116521 (in German)

Cremer, C. et al., German Patent Application No. 196.54.824.1/DE, submitted Dec 23, 1996, European Patent EP 1997953660, 08.04.1999, Japanese Patent JP 1998528237, 23.06.1999, United States Patent US 09331644, 25.08.1999

Cremer, C., A.V. Failla and B. Albrecht. 2002. Far-field light microscopical method, system and computer program product for analysing at least one object having a subwavelength size. US Patent7, 298, 461, filed Oct. 9, 2002, date of patent Nov 20, 2007

Cremer, C., C. Zorn and T. Cremer. 1974. An ultraviolet laser microbeam for $257 \mathrm{~nm}$. Microsc. Acta 75: 331-337

Cremer, C. and T. Cremer. 1978. Considerations on a Laser-Scanning-Microscope with high resolution and depth of field. Microsc. Acta 81: 31-44

Cremer, C., A. von Ketteler, P. Lemmer, R. Kaufmann, Y. Weiland, P. Mueller, M. Hausmann, D. Baddeley and A. Amberger. 2010. Far field fluorescence microscopy of cellular structures @ molecular resolution, in: Nanoscopy and Multidimensional Optical Fluorescence Microscopy, edited by A. Diaspro. Taylor and Francis. Abingdon, Oxford, UK, pp. 3/1-3/35

Cremer, C., R. Kaufmann, M. Gunkel, S. Pres, Y. Weiland, P. Müller, T. Ruckelshausen, P. Lemmer, F. Geiger, S. Degenhard, C. Wege, N.A.W. Lemmermann, R. Holtappels, H. Strickfaden and M. Hausmann. 2011. Enhanced resolution Imaging of Biological Nanostructures by Spectral Precision Distance Microscopy (SPDM). Biotechnology Journal 6: 1037-1051

Cremer, C., P. Edelmann, H. Bornfleth, G. Kreth, H. Muench, H. Luz and M. Hausmann. 1999. Principles of Spectral Precision Distance confocal microscopy for the analysis of molecular nuclear structure. Handbook of Computer Vision and Applications, edited by B. Jähne, H. Haußecker, P. Geißler, Vol. 3, pp. 839-857

Cremer, T. and C. Cremer. 2001. Chromosome territories, nuclear architecture and gene regulation in mammalian cells. Nature Reviews Genetics 2: 292-301

Cseresnyes, Z., U. Schwarz and C.M. Green. 2009. Analysis of replication factories in human cells by super-resolution light microscopy. BMC Cell Biology 10:88 doi: $10.1186 / 1471-2121-10-88$

Czapski, S. 1910. Theorie der optischen Instrumente nach Abbe. O. Lummer and F. Reiche, Die Lehre von der Bildentstehung im Mikroskop von Ernst Abbe, Friedrich View und Sohn, Braunschweig 
Davidovits, P. and M.D. Egger. 1971. Scanning Laser Microscope for Biological Investigations. Appl. Optics 10: 1615-1619

Dekker, A.J. and A. Van den Bos. 1997. Resolution: a survey. J. Opt. Soc. Am. A 14: 547-55

Dertinger, T., R. Colyer, G. Iyer, S. Weiss and J. Enderlein. 2009. Fast, background-free, 3D super-resolution optical fluctuation imaging (SOFI). Proc. Natl. Acad. Sci. 106: 2228722292

Dertinger, T., R. Colyer, R. Vogel, J. Enderlein and S. Weiss. 2010a. Achieving increased resolution and more pixels with superresolution optical fluctuation imaging (SOFI). Optics Express 18: 18875-18885

Dertinger, T., M. Heilemann, R. Vogel, M. Sauer and S. Weiss. 2010b. Superresolution optical fluctuation imaging with organic dyes. Angew. Chemie. Int. Ed. 49: 9441-9443

Dickson, R.M., A.B. Cubitt, R.Y. Tsien and W.E. Moerner. 1997. On/off blinking and switching behaviour of single molecules of green fluorescent protein. Nature 388: 355-358

Dierolf, M., A. Menzel, P. Thibault, P. Schneider, C.M. Kewish, R. Wepf, O. Bunk and F. Pfeiffer. 2010. Ptychographic X-ray computed tomography at the Nanoscale. Nature 467: 436-439

Dietzel, S., E. Weilandt, R. Eils, C. Münkel, C. Cremer and T. Cremer. 1995. Threedimensional distribution of centromeric or paracentromeric heterochromatin of chromosomes $1,7,15$, and 17 in human lymphocyte nuclei studied with light microscopic axial tomography. Bioimaging 3: 121-133

Donnert, G., J. Keller, R. Medda, M.A. Andrei, S.O. Rizzoli, R. Lührmann, R. Jahn, C. Eggeling and S.W. Hell. 2006. Macromolecular-scale resolution in biological fluorescence microscopy. Proc. Natl. Acad. Sci. USA 103: 11440-11445

Donnert, G., J. Keller, C.A. Wurm, S.O. Rizzoli, V. Westphal, A. Schönle, R. Jahn, S. Jakobs, C. Eggeling and S.W. Hell. 2007. Two-Color Far-Field Fluorescence Nanoscopy. Biophys. J. 92: L67-L69

Edelmann, P., A. Esa, M. Hausmann and C. Cremer. 1999. Confocal laser scanning microscopy: In situ determination of the confocal point-spread function and the chromatic shifts in intact cell nuclei. Optik 110: 194-198

Egner, A., M. Schrader and S.W. Hell. 1998. Refractive index mismatch induced intensity and phase variations in fluorescence confocal, multiphoton and 4Pi-microscopy. Opt. Commun. 153: 211-217

Egner, A., C. Geisler, C. von Middendorff, H. Bock, D. Wenzel, R. Medda, M. Andresen, A.C. Stiel, S. Jakobs, C. Eggeling, A. Schönle and S.W. Hell. 2007. Fluorescence nanoscopy in whole cells by asynchronous localization of photoswitching emitters. Biophysical Journal 93: $3285-3290$

Egner, A., S. Jakobs and S.W. Hell. 2002. Fast 100-nm resolution three-dimensional microscope reveals structural plasticity of mitochondria in live yeast. Proc. Natl. Acad. Sci. USA 99: 3370-3375

Egner, A., S. Verrier, A. Goroshkov, H.-D. Söling and S.W. Hell. 2004. 4Pi-microscopy of the Golgi apparatus in live mammalian cells. J. Struct. Biol. 147: 70-76

Esa, A., A.E. Coleman, P. Edelmann, S. Silva, C. Cremer and S. Janz. 2001. Conformational differences in the 3D-nanostructure of the immunoglobulin heavy-chain locus, a hotspot of chromosomal translocations in B lymphocytes. Cancer Genetics and Cytogenetics 127: $168-173$

Esa, A., P. Edelmann, L. Trakthenbrot, N. Amariglio, G. Rechavi, M. Hausmann and C. Cremer. 2000. 3D-spectral precision distance microscopy (SPDM) of chromatin nanostructures after triple-colour labelling a study of the BCR region on chromosome 22 and the Philadelphia chromosome. J. Microscopy 199: 96-105

Failla, A.V., and C. Cremer. 2001. Virtual Spatially Modulated Illumination Microscopy Prediction of Axial Distance Measurement. Proc. SPIE 4260: 120-125

Failla, A.V., B. Albrecht, U. Spoeri, A. Schweitzer, A. Kroll, M. Bach and C. Cremer. 2003. Nanotopology analysis using spatially modulated illumination (SMI) microscopy. Complexus 1: 29-40

Failla, A.V., A. Cavallo and C. Cremer. 2002a. Subwavelength size determination by spatially modulated illumination virtual microscopy. Appl. Optics 41: 6651-6659 
Failla, A.V., U. Spöri, B. Albrecht, A. Kroll and C. Cremer. 2002b. Nanosizing of fluorescent objects by spatially modulated illumination microscopy. Appl. Optics 41: 7275-7283

Fang, N., H. Lee, C. Sun and X. Zhang. 2005. Sub-Diffraction-Limited Optical Imaging with a Silver Superlens. Science 308: 534-537

Fölling, J., M. Bossi, H. Bock, R. Medda, C.A. Wurm, B. Hein, S. Jakobs, C. Eggeling and S.W. Hell. 2008. Fluorescence nanoscopy by ground-state depletion and single-molecule return. Nature Methods 5: 943-945

Frohn, J., H. Knapp and A. Stemmer. 2000. True optical resolution beyond the Rayleigh limit achieved by standing wave illumination. Proc. Natl. Acad. Sci. USA 97: 7232-7236

Gaskill, J.D. 1978. Linear Systems, Fourier Transforms, and Optics, John Wiley and Sons, New York, Vol. 38, pp. 165-169

Geisler, C., A. Schönle, C. von Middendorff, H. Bock, C. Eggeling, A. Egner and S.W. Hell. 2007. Resolution of $\lambda / 10$ in fluorescence microscopy using fast single molecule photoswitching. Applied Physics A: Materials Science and Processing 88: 223-226

Glaschick, S., C. Röcker, K. Deuschle, J. Wiedenmann, F. Oswald, V. Mailänder and G.U. Nienhaus. 2007. Axial Resolution Enhancement by 4Pi Confocal Fluorescence Microscopy with Two-Photon Excitation. J Biol Phys. 33: 433-443

Gordon, M.P., T. Ha and P.R. Selvin. 2004. Single-molecule high-resolution imaging with photobleaching. Proc. Natl. Acad. Sci. USA 101: 6462-6465

Gould, T.J. M.S. Gunewardene, M.V. Gudheti, V.V. Verkhusha, S. Yin, J.A. Gosse and S.T. Hess. 2008. Nanoscale Imaging of Molecular Positions and Anisotropies. Nature Methods 5: $1027-1030$

Grab, A.L. 2011. In situ Nachweis von Proteinadsorptionsprozessen mittels kombinierter Schwingquarzmikrowägung und Plasmonenresonanz in Nanopartikelfilmen sowie Detektion einzelner Alexa Moleküle mit SPDM. Diploma Thesis, Faculty of Physics \& Astronomy, University Heidelberg

Greger, K., M.J. Neetz, E.G. Reynaud and E.H. Stelzer. 2011. Three-dimensional Fluorescence Lifetime Imaging with a Single Plane Illumination Microscope provides an improved Signal to Noise Ratio. Optics Express 19: 20743-20750

Gross, L., F. Mohn, N. Moll, P. Liljeroth and G. Meyer. 2009. The chemical structure of a molecule resolved by atomic force microscopy, Science 325: 1110-1114

Grotjohann, T., I. Testa, M. Leutenegger, H. Bock, N.T. Urban, F. Lavoie-Cardinal, K.I. Willig, C. Eggeling, S. Jakobs and S.W. Hell. 2011. Diffraction-unlimited all-optical imaging and writing with a photochromic GFP. Nature 478: 204-208

Grüll, F., M. Kirchgessner, R. Kaufmann, M. Hausmann and U. Kebschull. 2011. Accelerating Image Analysis For Localization Microscopy With FPGAs. International Conference on Field Programmable Logic and Applications 2011, Chania, Greece, 2011

Gugel, H., J. Bewersdorf, S. Jakobs, J. Engelhardt, R. Storz and S.W. Hell. 2004. Cooperative 4Pi Excitation and Detection Yields Sevenfold Sharper Optical Sections in Live-Cell Microscopy. Biophys J. 87: 4146-4152

Gunkel, M., F. Erdel, K. Rippe, P. Lemmer, R. Kaufmann, C. Hörmann, C., R. Amberger and C. Cremer. 2009. Dual Color Localization Microscopy of Cellular Nanostructures. Biotechnology J. 4: 927-938

Gustafsson, M.G.L. 1999. Extended resolution fluorescence microscopy. Curr. Opinion Struct. Biol. 9: 627-634

Gustafsson, M.G.L. 2000. Surpassing the lateral resolution limit by a factor of two using structured illumination microscopy. J. Microsc. 198: 82-87

Gustafsson, M. 2005. Nonlinear structured-illumination microscopy: Wide-field fluorescence imaging with theoretically unlimited resolution. Proc. Natl. Acad. Sci. USA 102: 1308113086

Gustafsson, M., L. Shao, P.M. Carlton, C.J.R. Wang, I.N. Golubovskaya, W.Z. Cande, D.A. Agard and J.W. Sedat. 2008. Three-Dimensional Resolution Doubling in Wide-Field Fluorescence Microscopy by Structured Illumination. Biophysical Journal 94: 4957-4970

Ha, T., Th. Enderle, D.S. Chemla and S. Weiss. 1996. Dual-Molecule Spectroscopy: Molecular Rulers for the Study of Biological Macromolecules IEEE Journal of selected topics in Quantum Electronics 2: 1115-1128 
Hänninen, P.E., S.W. Hell, J. Salo, E. Soini and C. Cremer. 1995. Two-photon excitation $4 \mathrm{Pi}$ confocal microscope: Enhanced axial resolution microscope for biological research. Appl. Phys. Lett. 66: 1698-1700

Hausmann, M., B. Schneider, J. Bradl and C. Cremer. 1997. High-precision distance microscopy of 3D-nanostructures by a spatially modulated excitation fluorescence microscope. Proc. SPIE 3197: 217-222

Heilemann, M. 2008. Subdiffraction-resolution fluorescence imaging withconventional fluorescent probes. Angew. Chem. 47: 6172-6176

Heintzmann, R. 2003. Saturated patterned excitation microscopy with two-dimensional excitation patterns. Micron 34: 283-291

Heilemann, M., D.P. Herten, R. Heintzmann, C. Cremer, C. Müller, P. Tinnefeld, K.D. Weston, J. Wolfrum and M. Sauer. 2002. High-resolution colocalization of single dye molecules by fluorescence lifetime imaging microscopy. Analytical Chemistry 74: 35113517

Heintzmann, R. and C. Cremer. 1999. Lateral modulated excitation microscopy: Improvement of resolution by using a diffraction grating. Proc. SPIE 356: 185-196

Heintzmann, R. and C. Cremer. 2002. Axial tomographic confocal fluorescence microscopy. J. of Microscopy 206: 7-23

Heintzmann, R., T. Jovin and C. Cremer. 2002. Saturated patterned excitation microscopy a concept for optical resolution improvement. J. Opt. Soc. Am. A 19: 1599-1609

Hell, S. 1990a. "Abbildung transparenter Mikrostrukturen im Konfokalen Mikroskop" (Imaging of transparant microstructures in the confocal microscope), Ph.D. Dissertation Physics, University Heidelberg, Germany

Hell, S.W. 1990b. Double confocal microscope. European Patent 91121368 (July 2, 1992); German patent application P40 40441.2 (filed December 18, 1990; published 1992)

Hell, S.W. 2003. Toward fluorescence nanoscopy. Nature Biotechnology 2: 1347-1355

Hell, S.W. 2007. Far-Field Optical Nanoscopy, Science 316: 1153-1158

Hell, S.W. 2009. Microscopy and its focal switch. Nature Methods 6: 24-32

Hell, S.W. and M. Kroug. 1995. Ground-state-depletion fluorescence microscopy: A concept for breaking the diffraction resolution limit. Appl. Phys. B 5: 495-497

Hell, S.W. and M. Nagorni. 1998. 4Pi confocal microscopy with alternate interference. Optics Letters 23: 1567-1569

Hell, S. and E.H.K. Stelzer. 1992a. Properties of a 4Pi confocal fluorescence microscope. $J$. Opt. Soc. Am. A 9: 2159-2166

Hell, S. and E.H.K. Stelzer. 1992b. Fundamental improvement of resolution with a 4Piconfocal fluorescence microscope using two-photon excitation. Optics Communications 93: $277-282$

Hell, S.W. and J. Wichmann. 1994. Breaking the diffraction resolution limit by stimulated emission: stimulated-emission-depletion fluorescence microscopy. Optics Letters 19: 780782

Hell, S.W., S. Lindek, C. Cremer and E.H.K. Stelzer. 1994a. Measurement of the 4Pi-confocal point spread function proves $75 \mathrm{~nm}$ axial resolution. Applied Physics Letters 64: 13351337

Hell, S.W., E.H.K. Stelzer, S. Lindek and C. Cremer. 1994b. Confocal microscopy with an increased detection aperture: true-B 4Pi confocal microscopy. Optics Lett. 19: 222-224

Helmholtz, H. 1874. Die theoretische Grenze für die Leistungsfähigkeit der Mikroskope. Annalen der Physik, (Leipzig) Jubelband, 557-585

Herzenberg, L.A., D. Parks, B. Sahaf, O. Perez, M. Roederer and L.A. Herzenberg. 2002. Activated Cell Sorter and Flow Cytometry: A View from Stanford. Clinical Chemistry 48: $1819-1827$

Hess, S., T. Girirajan and M. Mason. 2006. Ultra-High Resolution Imaging by Fluorescence Photoactivation Localization Microscopy. Biophysical Journal 91: 4258-4272

Hess, S.T., T.J. Gould, M.V. Gudheti, S.A. Maas, K.D. Mills and J. Zimmerberg. 2007. Dynamic clustered distribution of hemagglutinin resolved at $40 \mathrm{~nm}$ in living cell membranes discriminates between raft theories. Proc. Natl. Acad. Sci. 104: 17370-17375 
Hildenbrand, G., A., Rapp, U. Spori, C. Wagner, C. Cremer and M. Hausmann. 2005. NanoSizing of Specific Gene Domains in Intact Human Cell Nuclei by Spatially Modulated Illumination Light Microscopy. Biophysical Journal 88: 4312-4318

Hofmann, M., C. Eggeling, S. Jakobs and S.W. Hell. 2005. Breaking the diffraction barrier in fluorescence microscopy at low light intensities by using reversibly photoswitchable proteins. Proc. Natl. Acad. Sci. USA 102: 17565-17569

Huang, B., W. Wang, M. Bates, X. Zhuang. 2008. Three-dimensional super-resolution imaging by stochastic optical reconstruction microscopy. Science 319: 810-813

Huber, O., A. Brunner, P. Maier, R. Kaufmann, P.-O. Couraud, C. Cremer and G. Fricker. 2012. Localization microscopy (SPDM) reveals clustered formations of P-Glycoprotein in a human blood-brain barrier model. PLoS ONE 7 e44776: 1-10

Hüve, J., R. Wesselmann, M. Kahms and R. Peters. 2008. 4Pi microscopy of the nuclear pore complex. Biophysical J. 95: 877-885

Jones, S., S.-H. Shim, J. He, X. Zhuang. 2011. Fast three-dimensional super-resolution imaging of live cells. Nature Methods 8: 499-505

Jutamulia, S. 2002. Selected Papers on Near-Field Optics, SPIE Milestone Series, SPIE Optical Engineering Press, Bellingham, Vol. MS 172

Kaufmann, R., P. Lemmer, M. Gunkel, Y. Weiland, P. Müller, M. Hausmann, D. Baddeley, R. Amberger and C. Cremer. 2009. SPDM-Single Molecule Superresolution of Cellular Nanostructures. Proc. SPIE 7 185: 71850J-1-71850-19

Kaufmann, R., P. Müller, M. Hausmann and C. Cremer. 2011a. Analysis of Her2/neu membrane protein clusters in different types of breast cancer cells using localization microscopy. J. of Microscopy 242: 46-54

Kaufmann, R., P. Müller, M. Hausmann and C. Cremer. 2011b. Nanoimaging cellular structures in label-free human cells by spectrally assigned localization microscopy. Micron 42: 348-352

Kaufmann, R., J. Piontek, F. Grüll, M. Kirchgessner, J. Rossa, H. Wolburg, I.E. Blasig and C. Cremer. 2012a. Visualization and quantitative analysis of reconstituted tight junctions using localization microscopy. PLoS ONE 7 e31128: 1-9

Kaufmann, R., C. Cremer and J.G. Gall. 2012b. Superresolution imaging of transcription units on newt lampbrush chromosomes, Chromosome Research, doi: $10.1007 / \mathrm{s} 10577-012-9306-z^{-}$

Keller, P.J., F. Pampaloni, and E.H. Stelzer. 2007. Three-dimensional preparation and imaging reveal intrinsic microtubule properties. Nature Methods 4: 843-846

Keller, P.J., A.D. Schmidt, J. Wittbrodt, E.H. Stelzer. 2008. Reconstruction of zebrafish early embryonic development by scanned light sheet microscopy. Science 322: 1065-1069

Klein, T., A. Löschberger, S. Proppert, S. Wolter, S. van de Linde, M. Sauer. 2011. Live-cell dSTORM with SNAP-tag fusion proteins. Nature Methods 8: 7-9

Köhler, H. 1981. On Abbe's theory of image formation in the microscope. Optica Acta 28: 1691-1701

Lacoste, T.D., X. Michalet, F. Pinaud, D.S. Chemla, A.P. Alivisatos and S. Weiss. 2000. Ultrahigh-resolution multicolor colocalization of single fluorescent probes. Proc. Natl. Acad. Sci. USA 97: 9461-9466

Lang, M., T. Jegou, I. Chung, K. Richter, S. Münch, A. Udvarhelyi, C. Cremer, P. Hemmerich, J. Engelhardt, S.W. Hell and K. Rippe. 2010. Three-dimensional organization of promyelocytic leukemia nuclear bodies. J. of Cell Science 123: 392-412

Lanni, F., B. Bailey, D.L. Farkas and D.L. Taylor. 1993. Excitation field synthesis as a means for obtaining enhanced axial resolution in fluorescence microscopes. Bioimaging 1: 187-196

Lemmer, P., M. Gunkel, D. Baddeley, R. Kaufmann, A. Urich, Y. Weiland, J. Reymann, P. Müller, M. Hausmann and C. Cremer. 2008. SPDM: Light microscopy with singlemolecule resolution at the nanoscale. Applied Physics B 93: 1-12

Lemmer, P., M. Gunkel, D. Baddeley, R. Kaufmann, Y. Weiland, P. Müller, A. Urich, R. Amberger, H. Eipel, M. Hausmann and C. Cremer. 2009. Using Conventional Fluorescent Markers for Far-field Fluorescence Localization Nanoscopy allows Resolution in the $10 \mathrm{~nm}$ Regime. J. of Microscopy 235: 163-171 
Lewis, A., M. Isaacson, A. Harootunian and A. Muray. 1984. Development of a $500 \AA$ spatial resolution light microscope. I. Light is efficiently transmitted through $\lambda / 16$ diameter apertures. Ultramicroscopy 13: 227-231

Lidke, K.A., B. Rieger, T.M. Jovin and R. Heintzmann. 2005. Superresolution by localization of quantum dots using blinking statistics. Opt. Express 13: 7052-7062

Lindek, S., C. Cremer and E.H.K. Stelzer. 1994. Theta microscopy allows phase regulation in 4Pi(A)-confocal two-photon fluorescence microscopy. Optik 98: 15-20

Lindek, S., C. Cremer and E.H.K. Stelzer. 1996. Confocal theta fluorescence microscopy with annular apertures. Appl. Optics 35: 126-130

Lipson, A., S.G. Lipson, H. Lipson. 2010. Optical Physics, 4th edn., Cambridge University Press, Cambridge

Liu, Y. and X. Zhang. 2011. Metamaterials: a new frontier of science and technology. Chem. Soc. Rev. 40: 2494-2507

Löschberger, A., S. van de Linde, M.-C. Dabauvalle, B. Rieger, M. Heilemann, G. Krohne and M. Sauer. 2012. Super-resolution imaging visualizes the eightfold symmetry of gp210 proteins around the nuclear pore complex and resolves the central channel with nanometer resolution. J. of Cell Science 125: 570-575

Lukosz, W. 1966. Optical systems with resolving powers exceeding the classical limit, Part 1. J. Opt. Soc. Am. 56: 1463-1472

Lukosz, W. 1967. Optical systems with resolving powers exceeding the classical limit. II. $J$. Opt. Soc. Am. 57: 932-941

Lummer, O. and F. Reiche. 1910. Die Lehre von der Bildentstehung im Mikroskop von Ernst Abbe, Friedrich View und Sohn, Braunschweig

Mahajan, V.N. 2001. Optical Imaging and Aberrations, Part II, Wave Diffraction. SPIE Press, Bellingham

Markaki, Y., M. Gunkel, L. Schermelleh, S. Beichmanis, J. Neumann, M. Heidemann, H. Leonhardt, D. Eick, C. Cremer and T. Cremer. 2011. Functional nuclear organization of transcription and DNA replication: a topographical marriage between chromatin domains and the interchromatin compartment. Cold Spring Harbor Symposia on Quantitative Biology 75: 1-18, doi:10.1101/sqb.2010.75.042

Martin, Y. 1995. Selected Papers on Scanning Probe Microscopes, SPIE Milestone Series, SPIE Optical Engineering Press, Bellingham, Vol. MS 107

Martin, S., A.V. Failla, U. Spoeri, C. Cremer and A. Pombo. 2004. Measuring the Size of Biological Nanostructures with Spatially Modulated Illumination Microscopy. Molecular Biology of the Cell 15: 2449-2455

Masters, B.R. 1996. Selected Papers on Confocal Microscopy, SPIE Milestone Series, volume MS 131, SPIE Optical Engineering Press, Bellingham

Masters, B.R. 2001. Selected Papers on Optical Low-Coherence Reflectometry \& Tomography, SPIE Milestone Series, SPIE Optical Engineering Press, Bellingham, Vol. MS 165

Masters, B.R. 2003. Selected Papers on Multiphoton Excitation Microscopy, SPIE Milestone Series, volume MS 175, SPIE Optical Engineering Press, Bellingham

Masters, B.R. 2007. Ernst Abbe and the Foundation of Scientific Microscopes, Optics and Photonics News, Optical Society of America, Washington, DC 18: 18-23

Masters, B.R. 2008. History of the Optical Microscope in Cell Biology and Medicine, in: Encyclopedia of Life Sciences (ELS), John Wiley and Sons, Ltd: Chichester, UK, doi: 10.1002/9780470015902.a0003082

Masters, B.R. 2009a. The Development of Fluorescence Microscopy, in: Encyclopedia of Life Sciences (ELS), John Wiley and Sons, Ltd: Chichester, UK,

DOI : 10.1002/9780470015902.a0022093

Masters, B.R. 2009b. History of the Electron Microscope in Cell Biology, in: Encyclopedia of Life Sciences (ELS), Chichester, UK. John Wiley and Sons, Ltd: September 2009, DOI : 10.1002/9780470015902.a0021539

Masters, B.R. 2010. Three-Dimensional Confocal Microscopy of the Living Human Cornea, in: Handbook of Optics, Vision and Vision Optics, 3rd edn., edited by M. Bass, J. M. Enoch, V. Lakshminarayanan, 17.1-17.11, McGraw-Hill, New York, Vol. III 
Masters, B.R. and P.T.C. So. 2008. Handbook of Biomedical Nonlinear Optical Microscopy, Oxford University Press, New York

Mathée, H., D. Baddeley, C. Wotzlaw, J. Fandrey, C. Cremer and U. Birk. 2006. Nanostructure of specific chromatin regions and nuclear complexes. Histochem. Cell Biology 125: 75-82

Mathee, H., D. Baddeley, C. Wotzlaw, C. Cremer and U. Birk. 2007. Spatially Modulated Illumination Microscopy using one objective lens. Optical Engineering 46: 083603/1083603/8

Matsuda, A., L. Shao, J. Boulanger, C. Kervrann, P.M. Carlton, P. Kner, D. Agard and J.W. Sedat. 2010. Condensed Mitotic Chromosome Structure at Nanometer Resolution Using PALM and EGFP- Histones. PLoS ONE 5 e12768: 1-12

McCutchen, C.W. 1967. Superresolution in microscopy and the Abbe resolution limit. J. Opt. Soc. Am. 57: 1190-1192

McEvoy, A.L., D. Greenfield, M. Bates and J. Liphardt. 2010. Single-molecule localization microscopy for biological imaging. BMC Biology 8: 106, http://www.biomedcentral.com/ $1741-7007 / 8 / 106$

McMullan, D. 1990. The prehistory of scanned image microscopy. Part 1: Scanned optical microscopes. Proceedings of the Royal Microscopical Society 25: 127-131

Müller, E.W. 1937. Beobachtungen über die Feldemission und die Kathodenzerstäubung an thoriertem Wolfram. Z. für Physik 106: 132-140

Müller, E.W. and T.T. Tsong. 1969. Field Ion Microscopy, Principles and Applications. American Elsevier Publishing Company, New York

Nagerl, U.V., K.I. Willig, B. Hein et al. 2008. Live-cell imaging of dendritic spines by STED microscopy. Proc. Natl. Acad. Sci. USA 105: 18982-18987

Nagorni, M. and S.W. Hell. 1998. 4Pi-Confocal Microscopy Provides Three-Dimensional Images of the Microtubule Network with 100- to 150-nm Resolution. J. Struct. Biol. 123: $236-247$

Newberry, S.P. and N.Y. Schenectady. 1959. X-ray Microscope. US Patent 2, 877, 353, granted March 10

Paesler, M.A. and P.J. Moyer. 1996. Near-field optics. Theory, Instrumentation, and Applications. John Wiley and Sons, Inc., New York

Pendry, J.B. 2000. Negative Refraction Makes a Perfect Lens, Phys. Rev. Lett. 85: 3966-3969

Pereira, C.F., J. Rossy, D. M. Owen, J. Mak and K. Gaus. 2012. HIV taken by STORM: Super-resolution fluorescence microscopy of a viral infection. Virology Journal 9: 84, doi: $10.1186 / 1743-422 X-9-84$

Perinetti, G., T. Müller, A. Spaar, R. Polishchuk, A. Luini and A. Egner. 2009. Correlation of $4 \mathrm{Pi}$ and electron microscopy to study transport through single Golgi stacks in living cells with super resolution. Traffic 10: 379-391

Pertsinidis, A., Y. Zhang and S. Chu. 2010. Subnanometre single-molecule localization, registration and distance measurements. Nature 466: 647-651

Pohl, D.W., W. Denk and M. Lanz. 1984. Optical stethoscopy: Image recording with resolution $\lambda / 20$. Appl. Phys. Lett. 44: 651-654

Porter, A.B. 1906. On the Diffraction Theory of Microscopic Vision. The London, Edinburgh, and Dublin Philosophical Magazine and Journal of Science 11: 154-166

Rauch, J., M. Hausmann, I. Solovei, B. Horsthemke, T. Cremer and C. Cremer. 2000. Measurement of local chromatin compaction by Spectral Precision Distance microscopy. in: Laser Microscopy, edited by K. Koenig, H.J. Tanke, H. Schneckenburger, Proc. SPIE 4164: $1-9$

Rauch, J., T.A. Knoch, I. Solovei, K. Teller, S. Stein, K. Buiting, B. Horsthemke, J. Langowski, T. Cremer, M. Hausmann and C. Cremer. 2008. Light-Optical precision measurements of the active and inactive Prader-Willi Syndrome imprinted regions in human cell nuclei. Differentiation 76: 66-83

Rayleigh, L. 1880a. Investigations in Optics, with special reference to the spectroscope. 1. Resolving or separating, power of optical instruments. The London, Edinburgh, and Dublin Philosophical Magazine and Journal of Science, VIII XXXI: 261-274 
Rayleigh, L. 1880b. On the resolving-power of telescopes. The London, Edinburgh, and Dublin Philosophical Magazine and Journal of Science X: 116-119

Rayleigh, L. 1896. On the Theory of Optical Images, with Special Reference to the microscope. The London, Edinburgh, and Dublin Philosophical Magazine and Journal of Science 42, Part XV: 167-195

Rayleigh, L. 1899. Investigations in optics, with special reference to the spectroscope. In: Scientific papers by John William Strutt, Baron Rayleigh, Cambridge, Cambridge University Press, Cambridge, 1899, Vol. 1, pp. 415-459

Reymann, J., D. Baddeley, M. Gunkel, P. Lemmer, W. Stadter, T. Jegou, K. Rippe, C. Cremer and U. Birk. 2008. High-precision structural analysis of subnuclear complexes in fixed and live cells via spatially modulated illumination (SMI) microscopy. Chromosome Research 16: 367-382

Ries, J., C. Kaplan, E. Platonova, H. Eghlidi and H. Ewers. 2012. A simple, versatile method for GFP-based super-resolution microscopy via nanobodies. Nature Methods 9: 582-584

Rittweger, E., K.Y. Han, S.E. Irvine, C. Eggeling and S.W. Hell. 2009. STED microscopy reveals crystal colour centres with nanometric resolution. Nature Photonics 3: 144-147

Rouquette, J., C. Cremer, T. Cremer and S. Fakan. 2010. Functional nuclear architecture studied by microscopy: state of research and perspectives. Int. Rev. Cell. Mol. Bio. 282: $1-90$

Ruska, E. 1979. Die frühe Entwicklung der Elektronenlisen und der Elektronmikroskopie, Acta Historica Leopoldina, 12, Halle/Saale, Deutsche Akademie der Naturforscher Leopoldina

Ruska, E. 1986. The development of the electron microscope and of electron microscopy. Nobel Lecture December 8, 1986

Rust, M., M. Bates and X. Zhuang. 2006. Sub-diffraction-limit imaging by stochastic optical reconstruction microscopy (STORM). Nature Methods 3: 793-795

Schellenberg, F.M. 2004. Selected Papers on Resolution Enhancement Techniques in Optical Lithography, SPIE Milestone Series, SPIE Press, Bellingham, Vol. MS 178

Schermelleh, L., P.M. Carlton, S. Haase, L. Shao, L. Winoto, P. Kner, B. Burke, M.C. Cardoso, D.A. Agard, M.G.L. Gustafsson, H. Leonhardt and J.W. Sedat. 2008. Subdiffraction multicolor imaging of the nuclear periphery with $3 \mathrm{~d}$ structured illumination microscopy. Science 320: 1332-1336

Schmahl, G. and D. Rudolph. 1989. X-ray microscope. US Patent 4, 870, 674

Schmidt, M., M. Nagorni and S.W. Hell. 2000. Subresolution axial distance measurements in far-field fluorescence microscopy with precision of 1 nanometer. Review of Scientific Instruments 71: 2742-2745

Schmidt, R., C.A. Wurm, S. Jakobs, J. Engelhardt, A. Egner and S.W. Hell. 2008. Spherical nanosized focal spot unravels the interior of cells. Nature Methods 5: 539-544

Schneider, G., P.G. Guttmann, S. Heim, S. Rehbein, F. Mueller, K. Nagashima, J.B. Heymann, W.G. Müller and J.G. McNally. 2010. Three-dimensional cellular ultrastructure resolved by X-ray microscopy. Nature Methods 7: 985-987

Schneider, B., B. Albrecht, P. Jaeckle, D. Neofotistos, S. Söding, Th. Jäger and C. Cremer. 2000. Nanolocalization measurements in spatially modulated illumination microscopy using two coherent illumination beams. Proc. SPIE 3921: 321-330

Schrader, M., F. Meinecke, K. Bahlmann, M. Kroug, C. Cremer, E. Soini and S.W. Hell. 1995. Monitoring the excited state of a dye in a microscope by stimulated emission. Bioimaging 3: 147-153

Schwentker, A., H. Bock, M. Hofmann, S. Jakobs, J. Bewersdorf, C. Eggeling and S.W. Hell. 2007. Wide-field subdiffraction RESOLFT microscopy using fluorescent protein photoswitching. Microscopy Research and Technique 70: 269-280

Schwering, M., A.A. Kiel, A. Kurz, K. Lymperopoulos, A. Sprödefeld, R. Krämer and D.P. Herten. 2011. Far-Field Nanoscopy with Reversible Chemical Reactions. Angew. Chem. Int. Edit. 50: 2940-2945

Sengupta, P., T. Jovanovic-Talisman, D. Skoko, M. Renz, S.L. Veatch and J. LippincottSchwartz. 2011. Probing protein heterogeneity in the plasma membrane using PALM and pair correlation analysis. Nature Methods 8: 969-975 
Sheppard, C.J.R. 2007. Fundamentals of superresolution. Micron 38: 165-169

Sheppard, C.J.R. and T. Wilson. 1978. Image formation in scanning microscopes with partially coherent source and detector. Optica Acta 25: 315-325

Shroff, H., C.G. Galbraith, J.A. Galbraith and E. Betzig. 2008. Live-cell photoactivated Localization Microscopy of nanoscale adhesion dynamics. Nature Methods 5: 417-423

Shtengel, G., J.A. Galbraith, C.G. Galbraith, J. Lippincott-Schwartz, J.M. Gillette, S. Manleyd, R. Sougrat, C.M. Watermane, P. Kanchanawong, M.W. Davidson, R.D. Fetter and H.F. Hess. 2009. Interferometric fluorescent super-resolution microscopy resolves 3D cellular ultrastructure. Proc. Natl. Acad. Sci. USA 106: 3125-3130

Sinnecker, D., P. Voigt, N. Hellwig and M. Schaefer. 2005. Reversible photobleaching of enhanced green fluorescent proteins. Biochemistry 44: 7085-7094

Smolyaninov, I.I., Y.-J. Hung and C.C. Davis. 2007. Magnifying Superlens in the Visible Frequency Range. Science 315: 1699-1701

Sparrow, C.M. 1916. On spectroscopic resolving power. The Astrophysical Journal XLIV: $76-86$

Staier, F., H. Eipel, P. Matula, A.V. Evsikov, M. Kozubek, C. Cremer and M. Hausmann. 2011. Micro Axial Tomography: a miniaturized, versatile stage device to overcomeresolution anisotropy in fluorescence light microscopy. Rev. Sci. Instr. 82: 09370. doi: $10.1063 / 1.3632115$

Steinhauer, C., C.C. Forthmann, J. Vogelsang and P. Tinnefeld. 2008. Super resolution Microscopy on the Basis of Engineered Dark States. J. Amer. Chemical Soc. 130: 1684016841

Stelzer, E.H.K. 2002. Beyond the diffraction limit? Nature 417: 806-807

Stelzer, E.H., I. Wackerand, J.R. De Mey. 1991. Confocal fluorescence microscopy in modern cell biology. Semin. Cell Biol. 2: 145-52

Strehl, K. 1894. Die Theorie des Fernrohrs auf Grund der Beugung des Lichts. JA Barth, Leipzig

Synge, E.H. 1928. A suggested method for extending microscopic resolution into the ultramicroscopic region. The London, Edinburgh, and Dublin Philosophical Magazine and Journal of Science 6: 356-362

Toraldo di Francia, G. 1955. Resolving power and information. J. Opt. Soc. Am. 45: 497-501

Travis J Gould, T.J., M.S. Gunewardene, M.V. Gudheti, V.V. Verkhusha, S.-R. Yin, J.A. Gosse and S.T. Hess. 2008. Knowledge on nanoscale imaging of molecular positions and anisotropies. Nature Methods 5: 1027-1030

Tykocinski, L.-O., A. Sinemus, E. Rezavandy, Y. Weiland, D. Baddeley, C. Cremer, S. Sonntag, K. Willeke, J. Derbinski and B. Kyewski. 2010. Epigenetic regulation of promiscuous gene expression in thymic medullary epithelial cells. Proc. Natl. Acad. Sci. USA 107: 19426-19431

Van Aert, S., A.J. den Dekker, D. Van Dyck and A. Van den Bos. 2007. The Notion of Resolution, in: Science of Microscopy, edited by Peter W. Hawkes, John C. H. Spence, Springer, New York, Vol. II

Van Aert, S., D. Van Dyck and A.J. den Dekker. 2006. Resolution of coherent and incoherent imaging systems reconsidered - Classical criteria and a statistical alternative. Optics Express 14: 3830-3839

van de Linde, S., A. Löschberger, T. Klein, M. Heidbreder, S. Wolter, M. Heilemann and Markus Sauer. 2011. Direct stochastic optical reconstruction microscopy with standard fluorescent probes. Nature Protocols 6: 991-1009

van Oijen, A.M., J. Köhler, J. Schmidt, M. Müller and G.J. Brakenhoff. 1998. 3-Dimensional super-resolution by spectrally selective imaging. Chemical Physics Letters 192: 182-187

Veselago, V.G. 1968. The electrodynamics of substances with simultaneously negative values of permittivity and permeability. Sov. Phys. Usp. 10: 509

Volkmann, H. 1966. Ernst Abbe, and His Work, Applied Optics 5: 1720-1731

Warren, W.S., H. Rabitz and M. Dahleh. 1993. Coherent Control of Quantum Dynamics: The Dream Is Alive. Science 259: 1581-1589

Westphal, V., S.O. Rizzoli, M.A. Lauterbach, D. Kamin, R. Jahn and S.W. Hell. 2008. Video-rate far-field optical nanoscopy dissects synaptic vesicle movement. Science $\mathbf{3 2 0}$ : 246-249 
Williams, C.S. and O.A. Becklund. 1989. Introduction to the Optical Transfer Function. Wiley-Interscience, New York

Willig, K.I., B. Harke, R. Medda and S.W. Hell. 2007. STED microscopy with continuous wave beams. Nature Methods 4: 915-918

Willig, K.I., S.O. Rizzoli, V. Westphal, R. Jahn and S.W. Hell. 2006. STED-microscopy reveals that synaptotagmin remains clustered after synaptic vesicle exocytosis. Nature 440: 935-939

Wombacher, R., M. Heidbreder, M. van de Linde, P. Sheetz, M. Heilemann, V.W. Cornish and M. Sauer. 2010. Live-cell super-resolution imaging with trimethoprim conjugates. Nature Methods 7: 717-719

Zhuang, X. 2009. Nano-imaging with Storm. Nature Photonics 3: 365-367

Zsigmondy, R. 1907. Über Kolloid-Chemie mit besonderer Berücksichtigung der anorganischen Kolloide, Verlag von Johann Ambrosius Barth, Leipzig

Zsigmondy, R. 1909. Colloids and the Ultramicroscope: A Manual of Colloid Chemistry and Ultramicroscopy, tr. Jerome Alexander, Wiley, New York

Open Access This is an open access article distributed under the terms of the Creative Commons Attribution License (http://creativecommons.org/licenses/by/3.0), which permits unrestricted use, distribution, and reproduction in any medium, provided the original work is properly cited. 